LHC Project Note 177

1999-01-12

(germana.riddone@cern.ch)

\title{
Preliminary risk analysis of the LHC cryogenic system
}

M. Chorowski*, Ph. Lebrun**, G. Riddone**

* Wroclaw University of Technology - Faculty of Mechanical and Power Engineering Institute of Heat Engineering and Fluid Mechanics

** LHC-ACR Group

\section{Summary}

The objective of the study is to identify all risks to personnel, equipment or environment resulting from cryogenic failures that may accidentally occur within the cryogenic system of Large Hadron Collider in any phase of the machine operation, and that could not be eliminated by design. We then formulate recommendations concerning lines of preventive and corrective defence, as well as further, more detailed studies. 


\section{Table of contents}

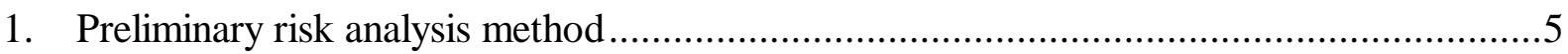

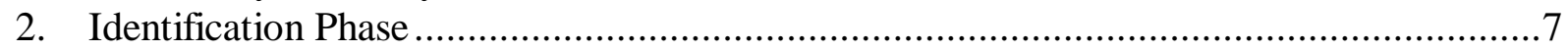

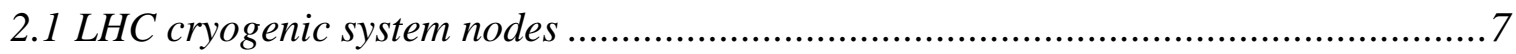

2.2 Failure modes of the LHC cryogenic system ..................................................... 10

2.3 Operation modes of the Large Hadron Collider .................................................. 15

3. Risks associated to failures at LHC cryogenic system nodes located in the tunnel. ..........18

3.1.LHC cryogenic system nodes located in the tunnel ................................................ 18

3.2. Potential and credible failures at the nodes located in the tunnel............................22

3.3. Analysis of and recommendations for credible failure modes at the nodes located in

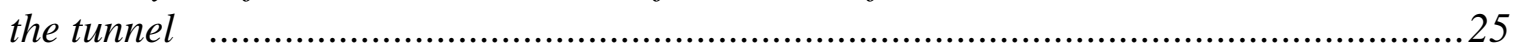

4. Risks associated to failures of LHC cryogenic system nodes in caverns and shafts ..........32

4.1 LHC cryogenic system nodes located in the caverns and shafts..............................32

4.2 Potential and credible failure modes at the nodes located in the caverns and shafts 37

4.3 Analysis of and recommendations for credible failure modes at the nodes located in

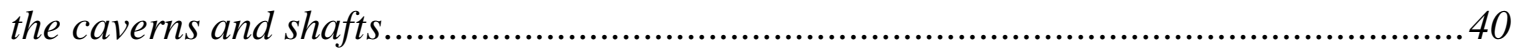

5. Risks associated at failures of LHC cryogenic system nodes at the surface.....................46

5.1 LHC cryogenic system nodes located at the surface (buildings and open space) ......46

5.2 Potential and credible failures at the nodes located at the surface ...........................51

5.3. Analysis of and recommendations for credible failure modes at the nodes located at

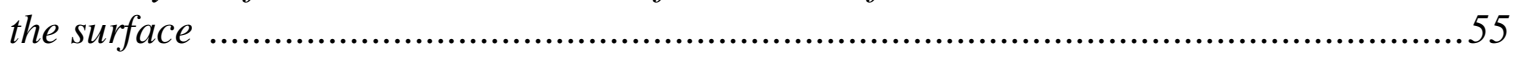

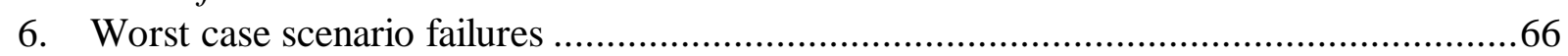

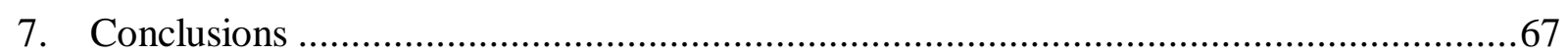

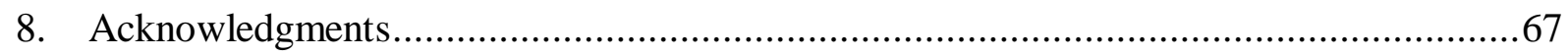

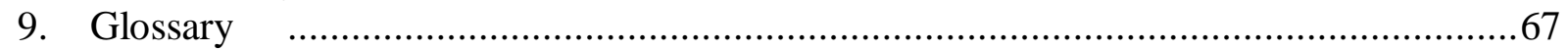

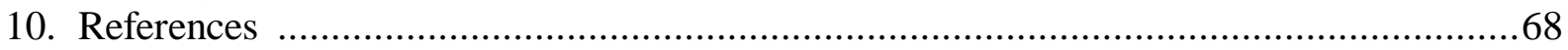




\section{List of tables}

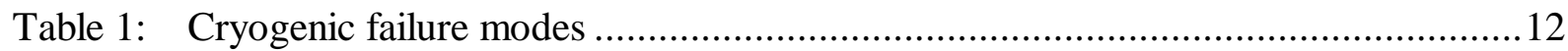

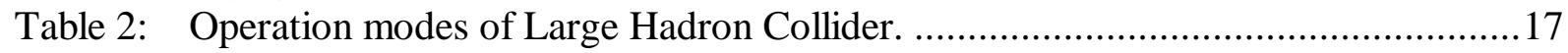

Table 3: LHC cryogenic system nodes located in the tunnel.......................................... 18

Table 4: Maximum He masses and volumes in the tunnel...........................................21

Table 5: Potential and credible (Y) failure modes of LHC nodes located in the tunnel (part 1)

Table 6: Credible failures of the cryogenic elements located in the tunnel and their causes ..25

Table 7: Recognized failures of the LHC elements located in the tunnel and their

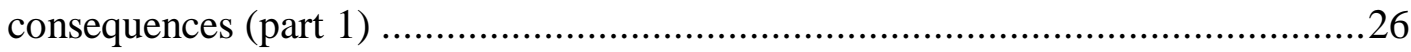

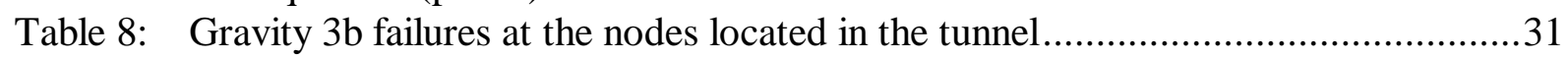

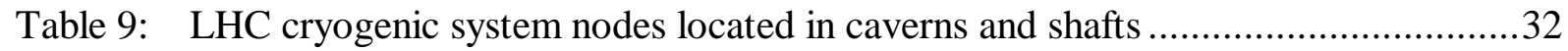

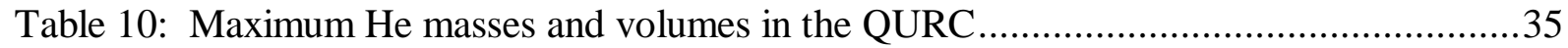

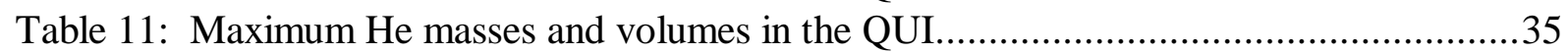

Table 12: Maximum He masses and volumes in the QURA..............................................35

Table 13: Maximum He masses and volumes in the insulated transfer lines and uninsulated

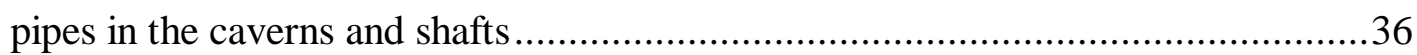

Table 14: Potential and credible (Y) failure modes of LHC nodes located in caverns.............38

Table 15: Potential and credible (Y) failure modes of LHC nodes located in the shafts..........39

Table 16: Credible failures of the machine elements located in caverns and shafts,and their

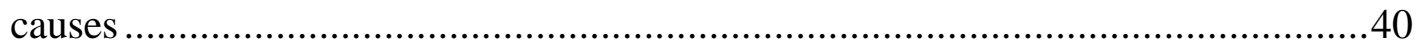

Table 17: Recognized failures of the LHC elements located in caverns and shafts, and their

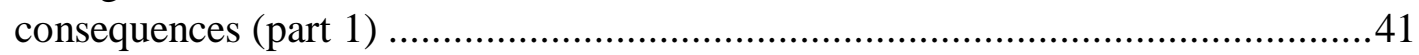

Table 18: Gravity $3 \mathrm{~b}$ failures at the nodes located in caverns and shafts ............................45

Table 19: LHC cryogenic system nodes located at the surface ..........................................46

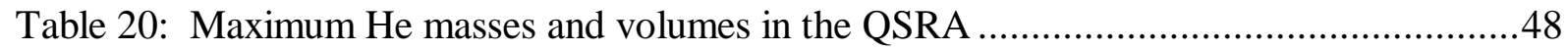

Table 21: Maximum He masses and volumes in the QSRB .............................................48

Table 22: Maximum He masses and volumes in the QSCA or QSCB ................................48

Table 23: Maximum He masses and volumes in the QSCC .............................................49

Table 24: Maximum helium and nitrogen masses and volumes in the storage vessels............49

Table 25: Maximum helium and nitrogen masses and volumes in interconnecting cryogenic lines and warm piping at the surface ...............................................................5. 50

Table 26: Potential and credible (Y) failure modes of LHC nodes located at the surface (part

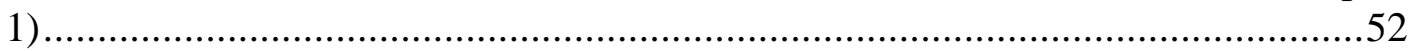

Table 27: Credible failures of the cryogenic elements located at the surface and their causes

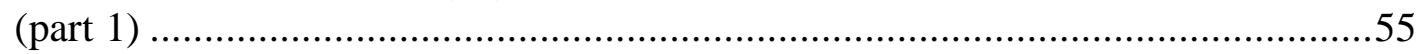

Table 28: Credible failure modes of the LHC elements located at the surface and their

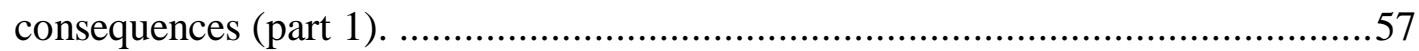

Table 29: Gravity $3 b$ failures of the nodes located in surface ..........................................65

Table 30: Worst case scenario failures for the LHC cryogenic system nodes located in the tunnel, caverns, shafts and at the surface. 


\section{List of figures}

Figure 1: Schematic representation of the risk analysis method ....................................6

Figure 2: General view of the LHC cryogenic system ....................................................

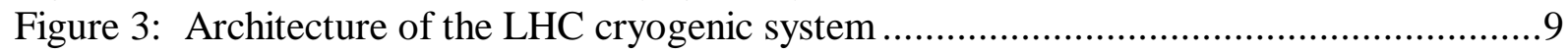

Figure 4: Flow-scheme of LHC elementary cooling loop ...............................................11

Figure 5: Simplified LHC flow-scheme showing safety relief valve locations.......................13

Figure 6: Generic flow (a) and technical (b) scheme of the LHC cryogenic system nodes with

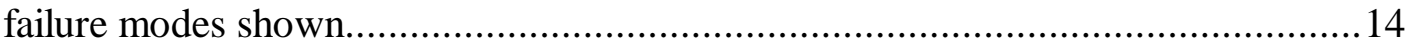

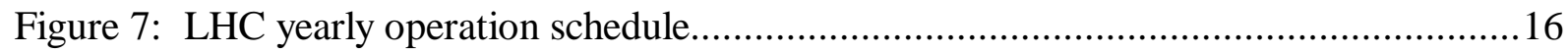

Figure 8: LHC machine components located in the tunnel. ............................................. 19

Figure 9: Vacuum and cold mass sub-sectorization (QRL and LHC machine cryostat) in the

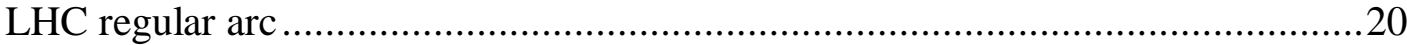

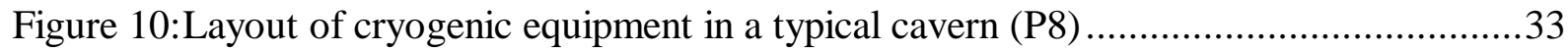

Figure 11:Typical layout of piping at an even-numbered point (P4, P6, P8) ........................34

Figure 12:Typical layout of cryogenic equipment at the surface (P8) ...............................47 


\section{Preliminary risk analysis method}

The Preliminary Risk Analysis (PRA) presented in this paper is performed in three steps, conceptually shown in Figure 1.

- The first step, called Identification is focused on listing of all the machine components, called later nodes, cryogenics-related failures and operation modes. Data have been collected through dedicated hearings, discussions and experience gathered at CERN.

- The objective of the second phase, called Combination is to list all potential failures for every node in any mode of the collider operation.

- $\quad$ The third phase, called Analysis is conducted in two steps. First for each potential failure a YES/NO decision is made to retain credible failures only. Then a descriptive analysis of the causes and consequences of each credible failure is made and a gravity number of 1 to 3 is assigned to each event. Failures of gravity 1 do not create any risk, neither to personnel nor to environment. For the failures of gravity 2 and 3, the associated risks are described and recommendations are formulated. Finally worst case scenarios for the nodes located in the tunnel, caverns, shafts and surface are identified and a descriptive probability for each of the mishaps is assigned.

The technique used for performing the PRA of the LHC cryogenic system is compatible with FEMECA (Failure Mode and Effect Criticality analysis) [1]. It fulfils the general requirements of Preliminary Risk Analysis of a complex technical system and in particular of a cryogenic system auxiliary to a large superconducting device [2, 3]. 


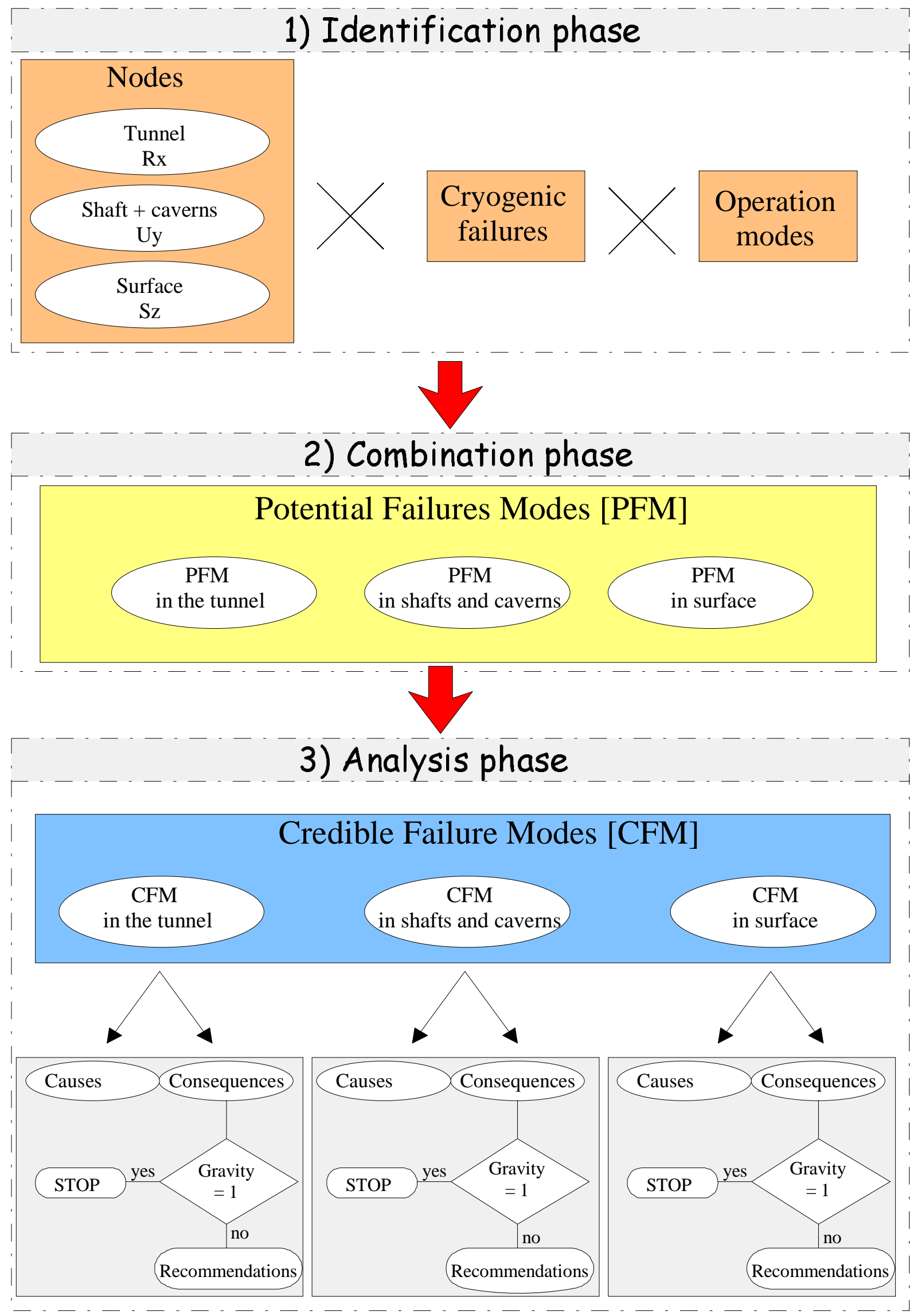

Figure 1: Schematic representation of the risk analysis method 


\section{Identification Phase}

\subsection{LHC cryogenic system nodes}

For the purpose of this analysis the cryogenic system of LHC is treated as composed of separate helium enclosures, called later on nodes. Each node is characterised by the amount and thermodynamic parameters of the helium enclosed. For the enclosures that are vacuum insulated, the volume of the vacuum space is the relevant parameter.

The general view of the LHC cryogenic system is shown in Figure 2, and its architecture in Figure 3. The LHC cryogenic nodes are classified according to their locations [4]:

- Nodes in tunnel, related later as R (e.g. LHC cryomagnets, cryogenic distribution line QRL),

- Nodes in caverns, related later as U (e.g. cold compressor boxes QURC),

- Nodes in shafts, related later as P (e.g. cryogenic interconnecting lines QPLA),

- Nodes at surface (buildings and free space), related later as $\mathrm{S}$ (e.g. helium compressor stations QSCA, QSCB, QSCC). 


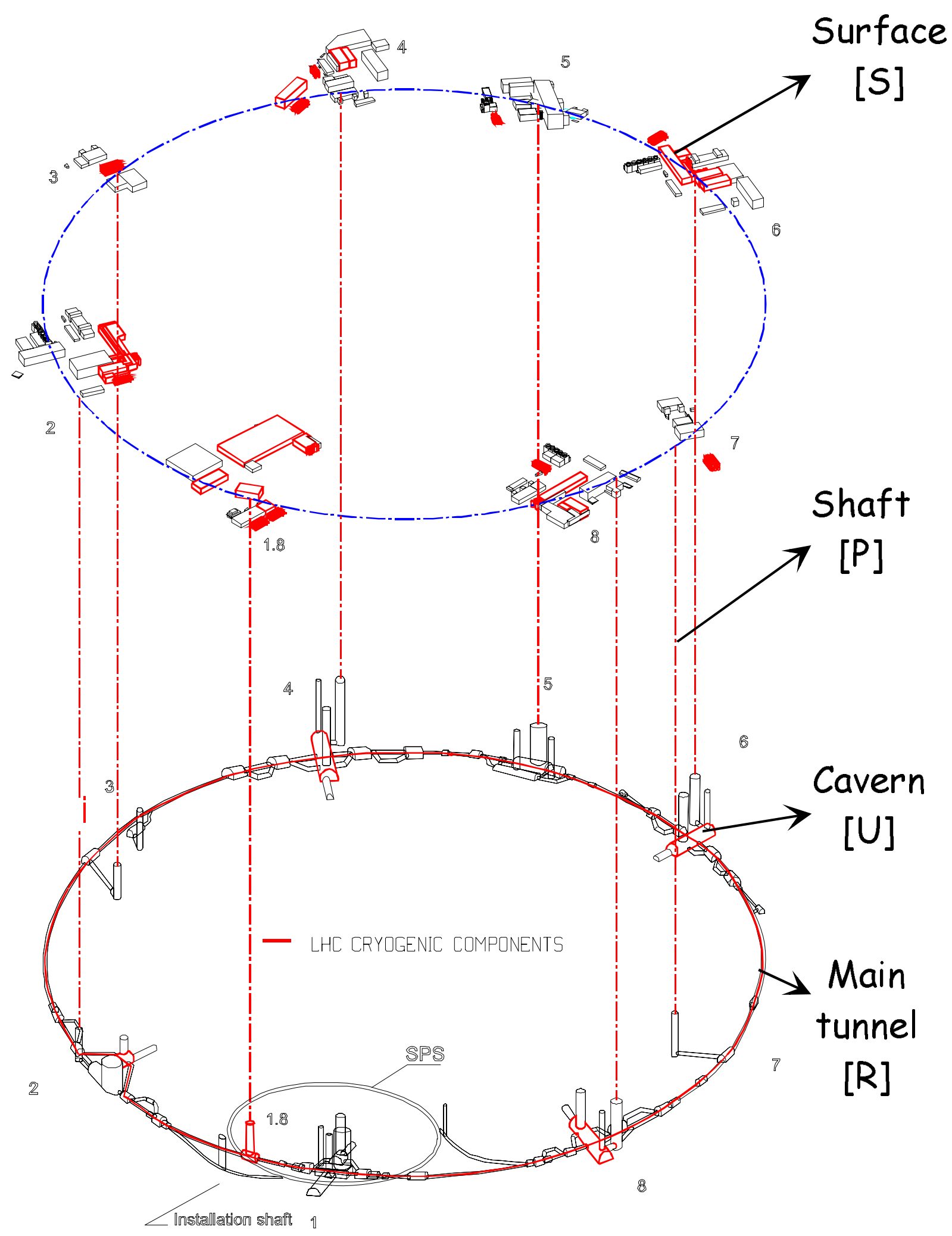

Figure 2: General view of the LHC cryogenic system 


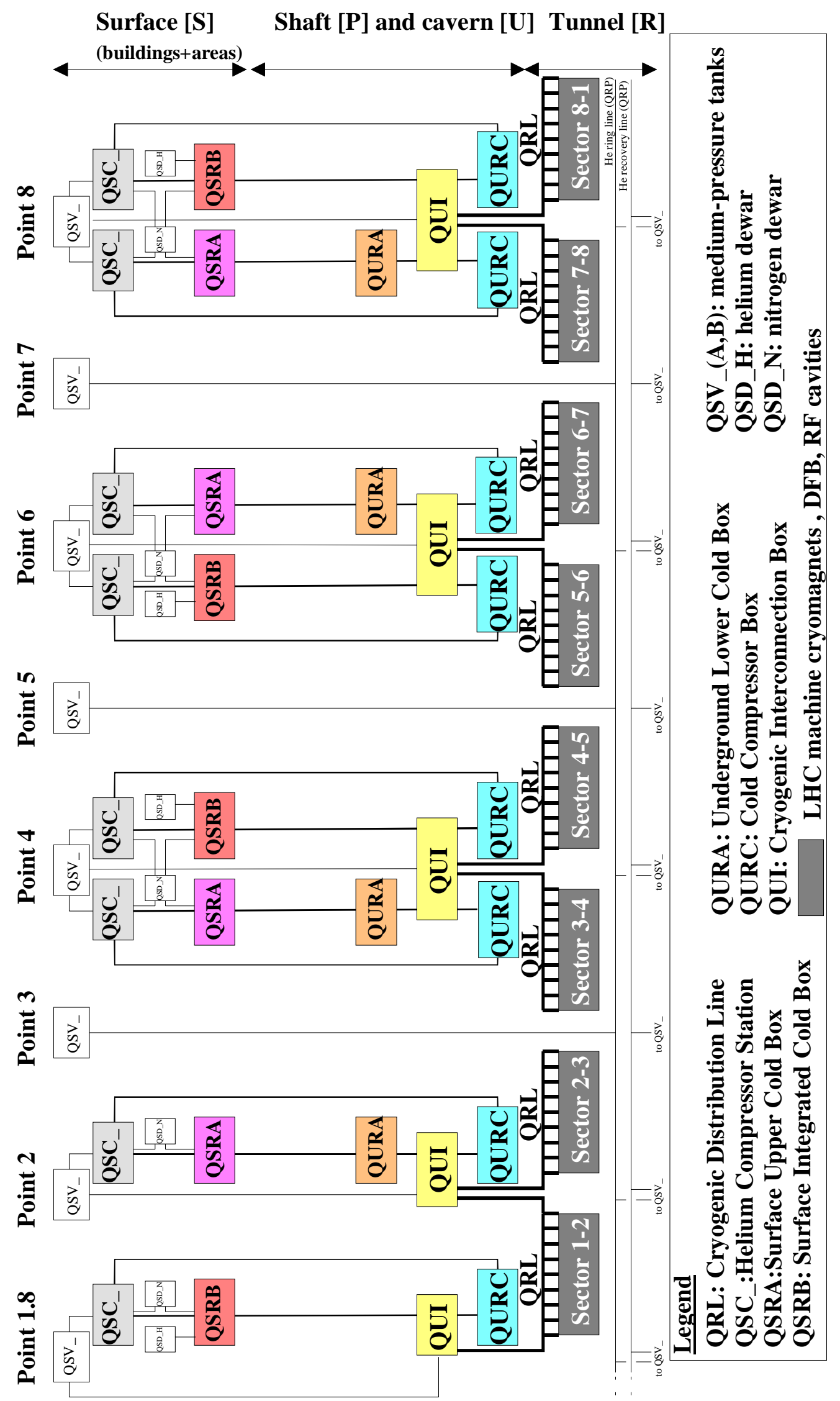

Figure 3: Architecture of the LHC cryogenic system 


\subsection{Failure modes of the LHC cryogenic system}

The safety philosophy of the LHC cryogenic system rests on some specific design features, listed in the following:

- the presence of a high-capacity, cold recovery header D which has a nominal working pressure of 1.3 bar (design pressure of $20 \mathrm{bar}$ ), a nominal working temperature of $20 \mathrm{~K}$ and a volume of about $60 \mathrm{~m}^{3}$ per sector. Helium expelled from the magnet cold mass after a loss of insulation vacuum or magnet resistive transitions will flow into header $\mathrm{D}$. The header will also accommodate helium relieved from other cryogenic distribution line QRL headers after insulation vacuum degradation (see Figure 4 and Figure 5 ). The only header protected with safety valves venting directly to caverns is header B because of low design pressure. A warm helium recovery line (see Figure 3) accommodates helium leaving the DFB after cooling the HTS current leads.

- the absence of nitrogen (liquid or gaseous) in the tunnel (see Figure 4 and Figure 5), in any operating mode. Liquid nitrogen is used only for helium pre-cooling at ground surface during cool-down.

- the absence of forced helium flow by mechanical devices (pumps) in cooling loops, resulting in quasi-static behaviour of fluid in case of header rupture (see Figure 4).

These features differ from the options chosen for the cryogenic systems of other large superconducting accelerators [5], and contribute to make the LHC cryogenic system inherently safe, with a limited number of credible risks and failures.

We define a cryogenic-related failure mode as an accidental event that involves helium or air mass transfer between helium enclosure, insulation or beam vacuum space and environment, in result of any constructional element break or malfunctioning (e.g. broken bellows, broken pipe or leaking valve). An unexpected energy release to cold mass helium as result of an extended magnet resistive transition or electrical arc is also a cryogenic failure mode.

We assume that within the LHC cryogenic system, only a single failure mode can occur at a given time.

A generic scheme of the LHC cryogenic system nodes is shown conceptually in Figure 6. Based on this scheme we can define the following potential failure modes for any of the system nodes (see Table 1). Depending on the node a list of potential failures may comprise all the events named in Table 1 (e.g. for LHC cryomagnets) or only a few of them (e.g. for mediumpressure helium storage tanks the only possible failure is helium flow to environment). 


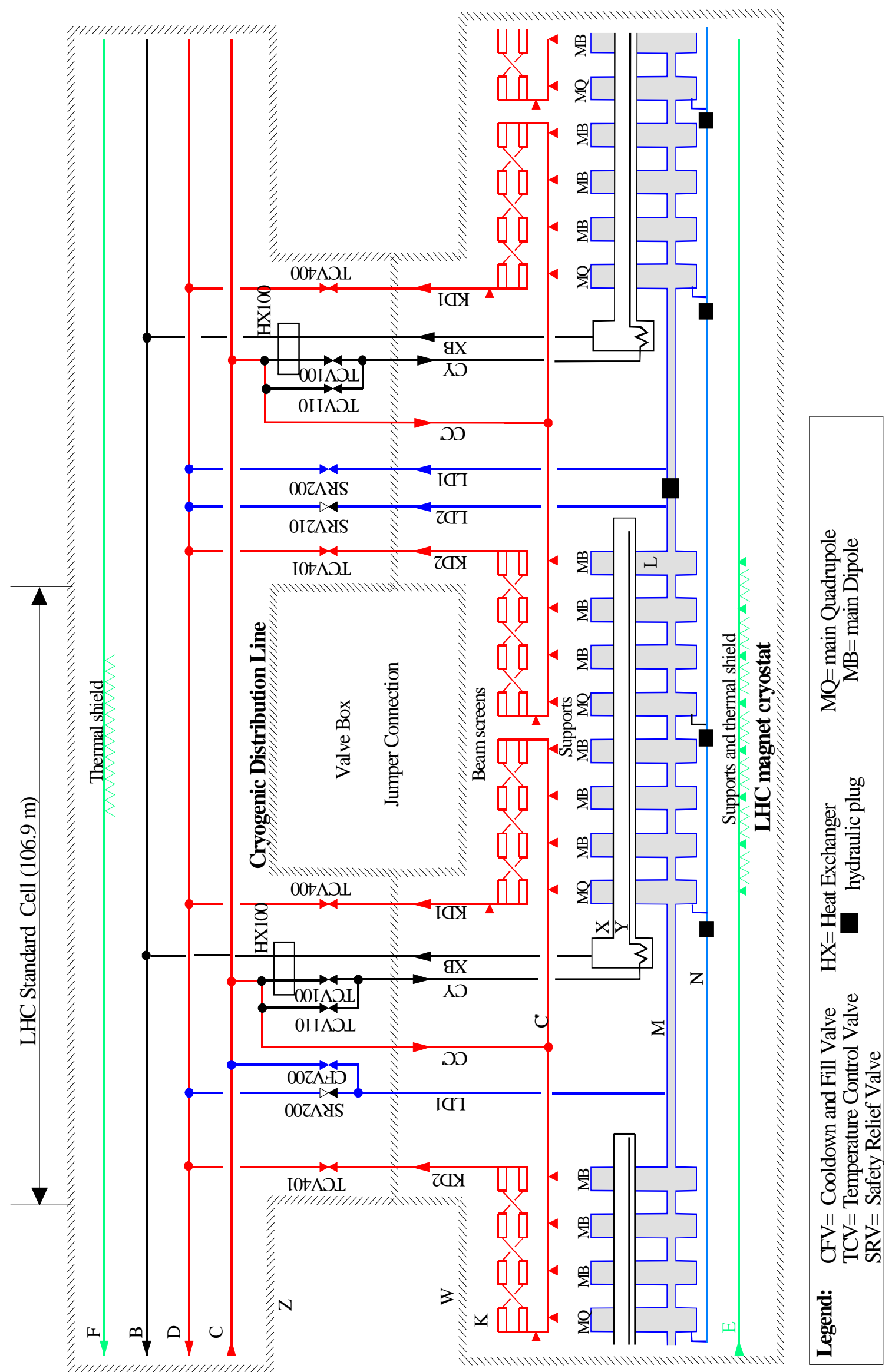

Figure 4: Flow-scheme of LHC elementary cooling loop 
Table 1: Cryogenic failure modes

\begin{tabular}{|c|l|}
\hline No. & Cryogenic failure \\
\hline 1 & Air flow to insulation vacuum \\
\hline $2^{(1)}$ & Helium flow to insulation vacuum \\
\hline 3 & Air flow to sub-atmospheric helium \\
\hline $4^{(1)}$ & Helium flow to environment \\
\hline 5 & Air flow to beam vacuum \\
\hline 6 & Helium flow to beam vacuum \\
\hline 7 & Pressurised helium flow to sub-atmospheric helium \\
\hline 8 & Energy release to cold mass helium due to a sector quench \\
\hline 9 & Energy release to cold mass helium due to electrical arc \\
\hline 10 & Oil flow to environment (in case of helium compressors only) \\
\hline
\end{tabular}

(1) in case of nitrogen tanks on surface the failure mode deals with "nitrogen flow" instead of "helium flow"

From their consequences, the LHC cryogenic related failures will be classified into three groups of different gravity, numbered from 1 to 3.

Gravity 1. Failure does not involve helium relief from the machine, or risk either to personnel or to environment.

Gravity 2. Helium ${ }^{(1)}$ is blown out of the machine directly to atmosphere at the surface.

Gravity 3. Helium ${ }^{(1)}$ is blown out of the machine to the confined area (tunnel, cavern, shaft, surface building):

Gravity $3 \mathrm{a}$ - while access to the tunnel is forbidden

Gravity $3 b$ - while access to the tunnel is allowed, under controlled conditions

According to the conclusions of AIWG (Access and Interlock Working Group), endorsed by the LHC Technical Committee (TCC Minutes 3.07.1998), access to the LHC will always be controlled. 

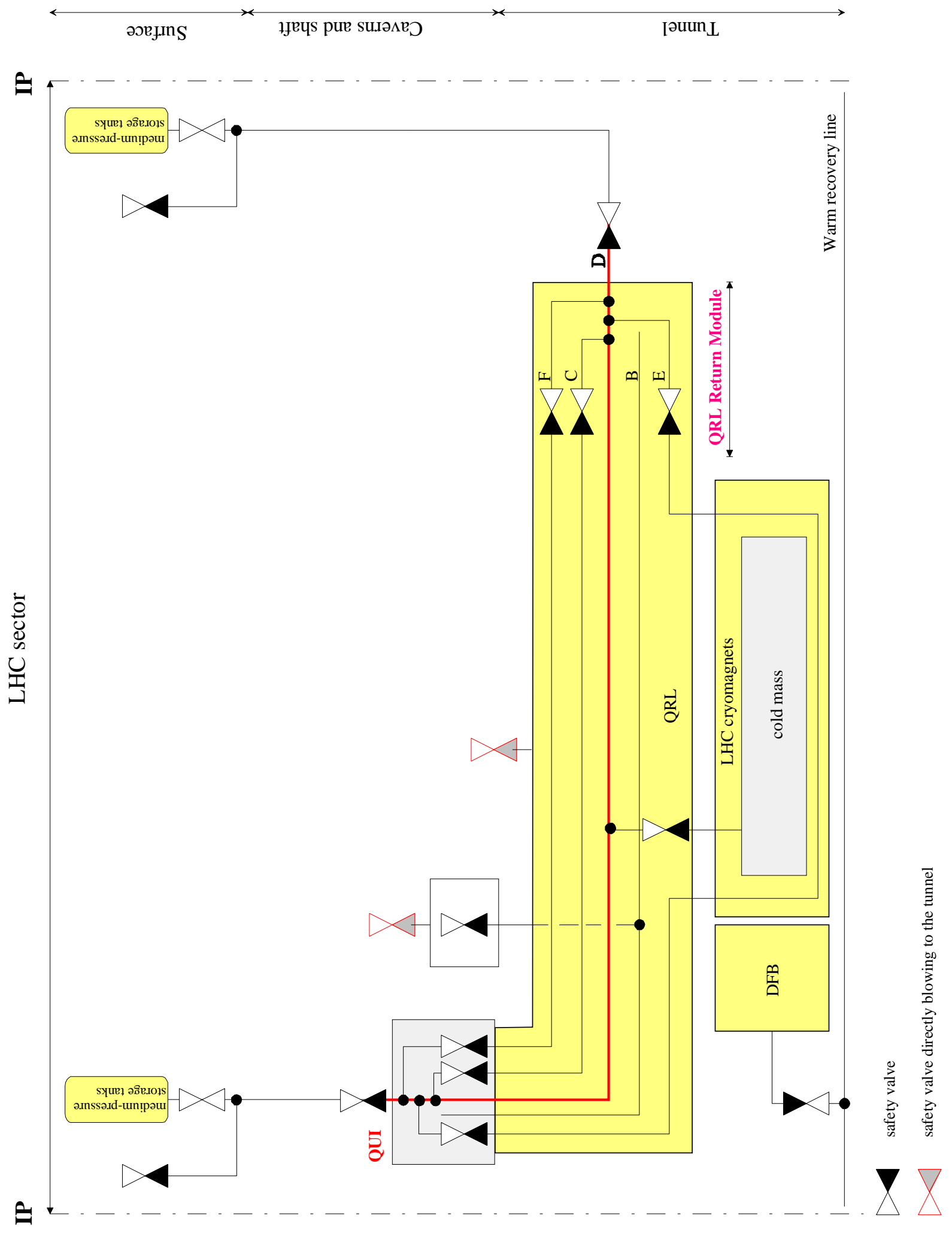

Figure 5: Simplified LHC flow-scheme showing safety relief valve locations 

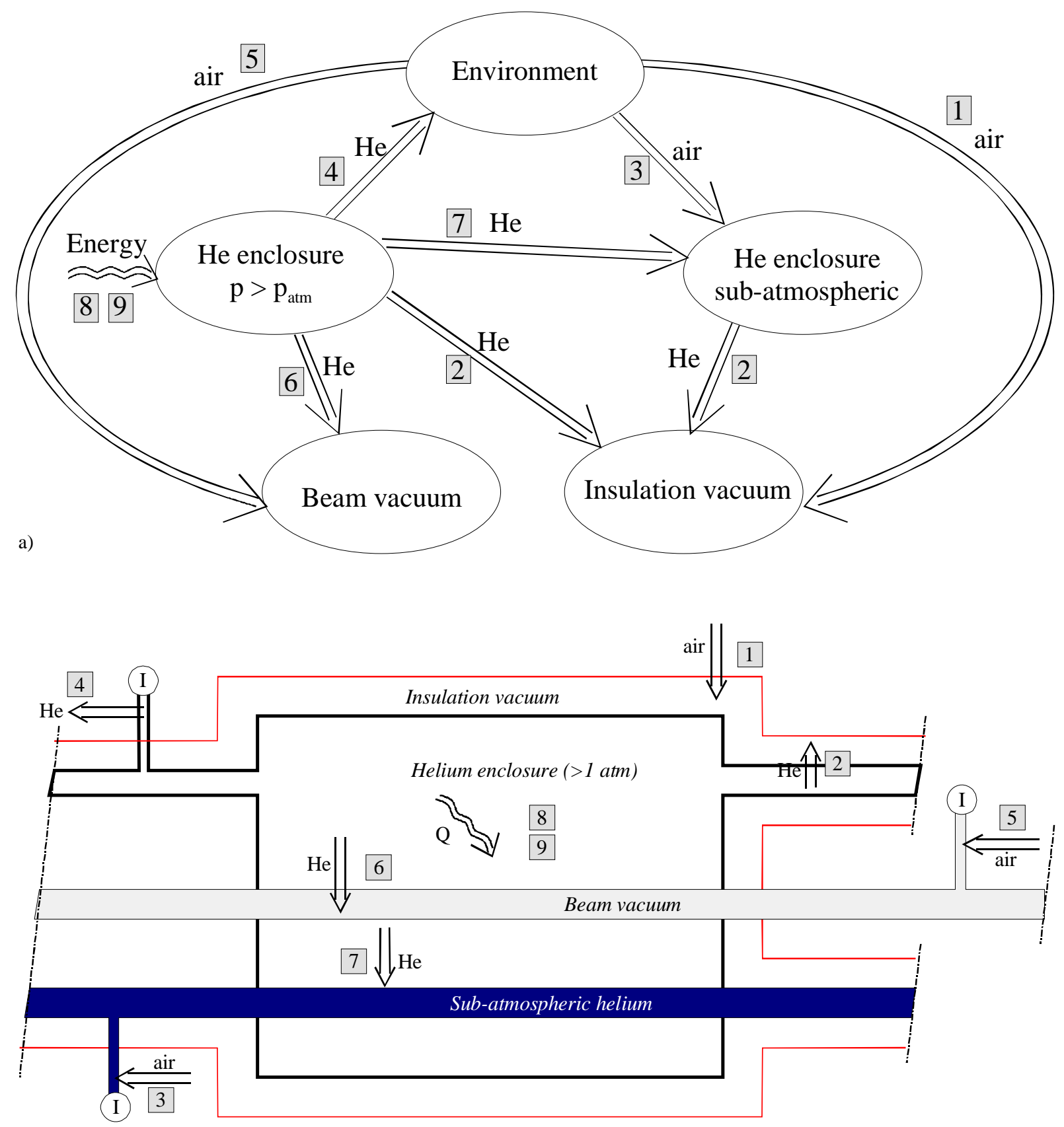

He/air $\Downarrow$ Mass transfer $\quad \mathrm{Q}\}$ Energy release

b)
3 Failure mode
(I) Instrumentation

Figure 6. Generic flow (a) and technical (b) scheme of the LHC cryogenic system nodes with failure modes shown. 


\subsection{Operation modes of the Large Hadron Collider}

The LHC operation modes have been defined on the basis of the tentative machine yearly operation schedule as shown in Figure 7 [6]. Additionally, the operation modes that will not happen on a regular basis have been taken into account. A list of LHC operation modes is given in Table 2. The helium temperature and the presence of magnetic field and beam define each operation mode. As a remark, the access condition to the tunnel is also given.

The part of a ring between two successive insertion points, which corresponds to $1 / 8$ of the machine circumference, is called a sector [7]. Cryogenic installations for each sector can be operated independently. During the machine installation and commissioning phases, different operation modes may be encountered even in adjacent sectors [8]. 


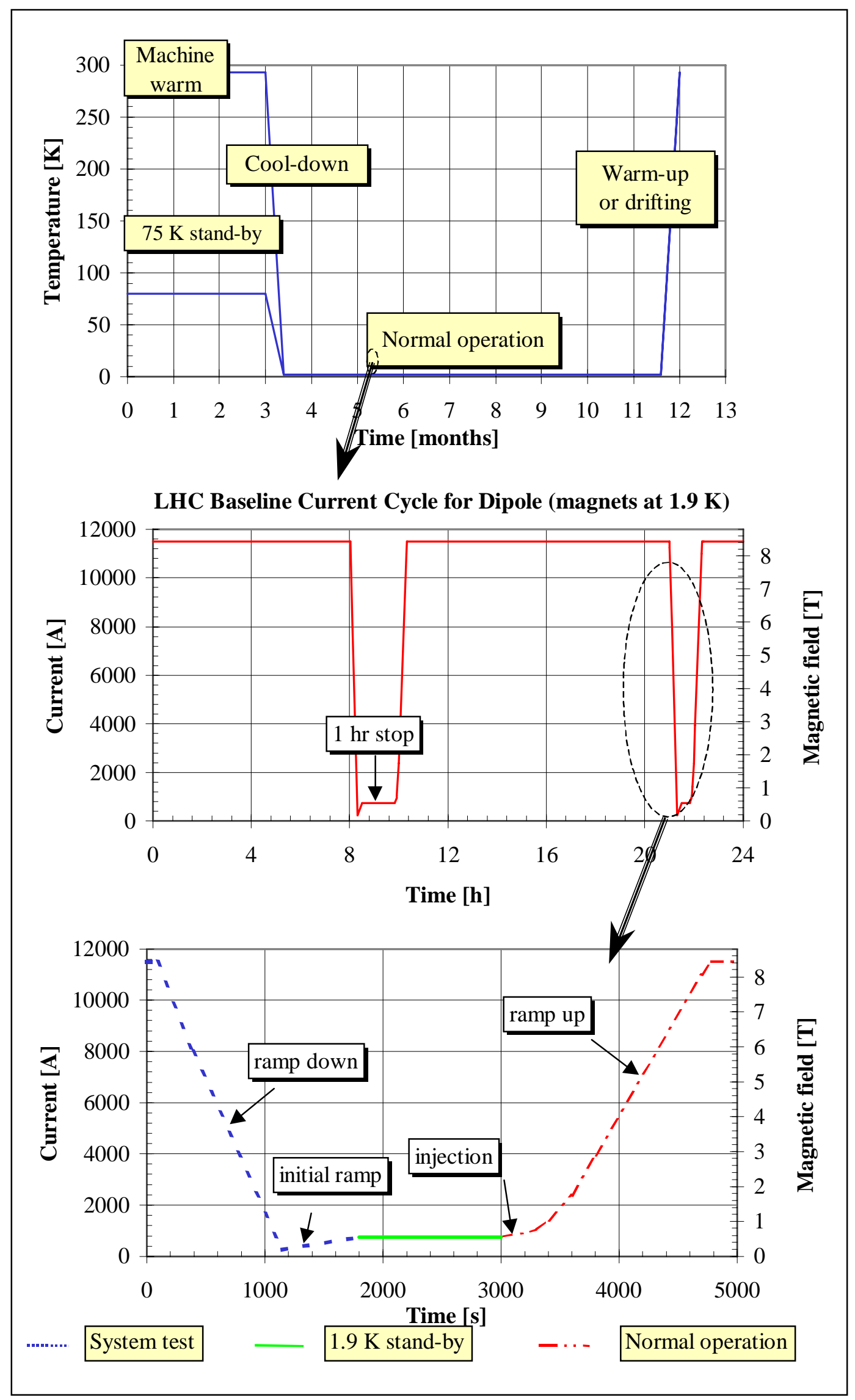

Figure 7: LHC yearly operation schedule 


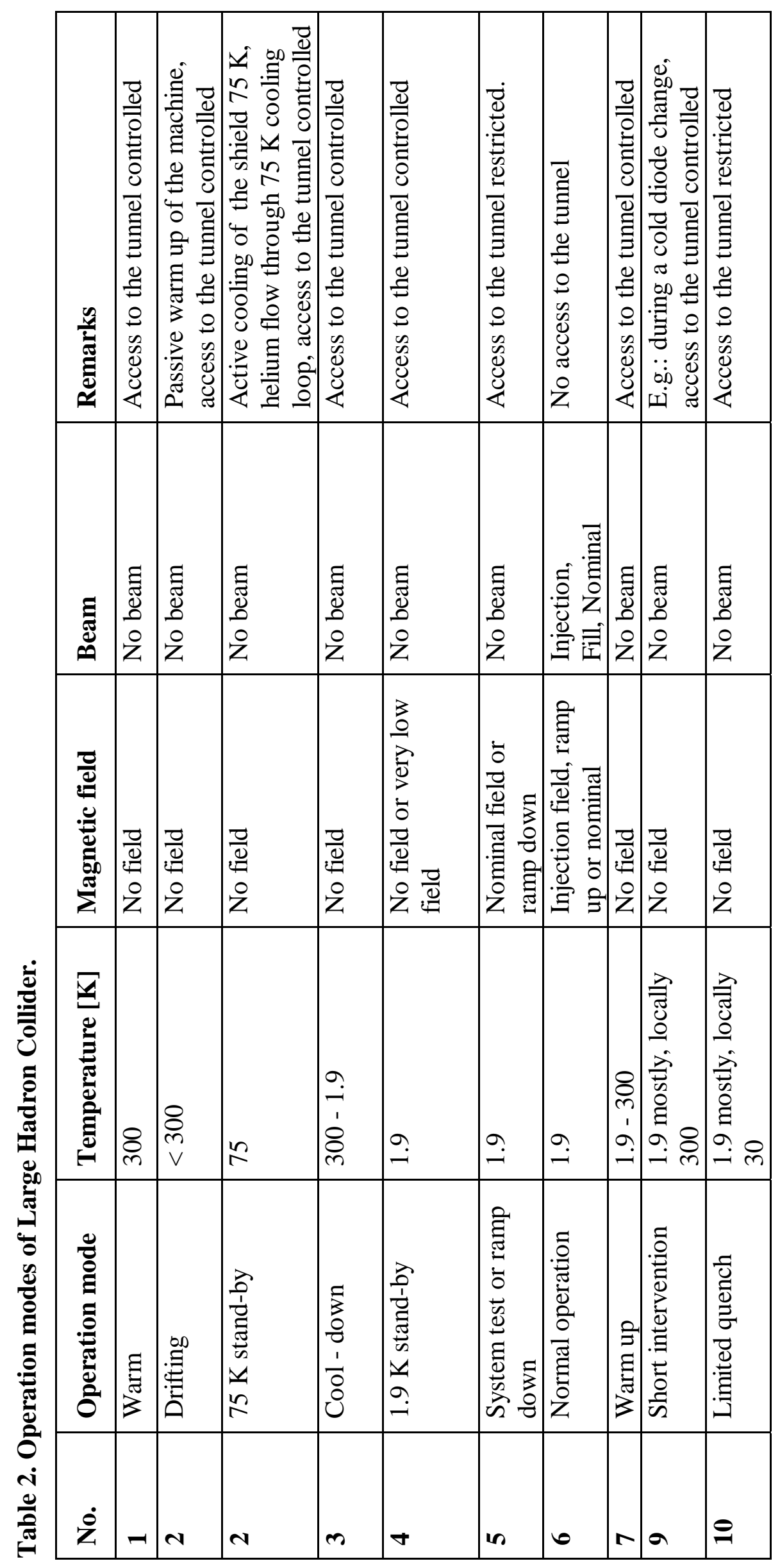




\section{Risks associated to failures at LHC cryogenic system nodes located in the tunnel.}

\subsection{LHC cryogenic system nodes located in the tunnel}

A typical LHC tunnel cross-section is shown in Figure 8. The accelerator will be composed of the LHC machine cryostat paralleled by the cryogenic distribution line QRL. Both will be linked by a jumper connection every $107 \mathrm{~m}$.

The nodes of LHC cryogenic system located in the tunnel are listed in Table 3.

Table 3. LHC cryogenic system nodes located in the tunnel.

\begin{tabular}{|l|l|l|}
\hline Node & Node description & Remarks \\
\hline R1 & LHC cryomagnets (including stand-alone units) & Along the machine \\
\hline $\mathbf{R 2}$ & $\begin{array}{l}\text { Cryogenic distribution line QRL (including jumper } \\
\text { connections) }\end{array}$ & Along the machine \\
\hline $\mathbf{R 3}$ & Helium ring line (QRP ; see figure 8) & Along the machine \\
\hline $\mathbf{R 4}$ & Warm helium recovery line (QRP ; see figure 8) & Along the machine \\
\hline R5 & Electrical feed box DFB & At the ends of each sector \\
\hline R6 & Radio-frequency RF cavities & $\begin{array}{l}\text { In straight sections, on } \\
\text { each side of PM4 }\end{array}$ \\
\hline
\end{tabular}

Each node located in the tunnel may be characterized by the amount of helium enclosed and vacuum insulation volume. The values depend on vacuum and cold mass sub-sectorization (see Figure 9). Based on the distances between QRL vacuum barriers (four cells), LHC machine cryostat vacuum barriers ( 2 cells) and cold mass tight plugs (two cells), the highest amount of helium and length of vacuum insulation involved in the failures are calculated and listed in Table 4 [9]. The operation modes when the helium quantities in the specified helium enclosures are the highest are: $1.9 \mathrm{~K}$ stand-by, Normal operation, System test and Ramp down. The exception is QRL header D, which has the highest helium density after a limited quench. 


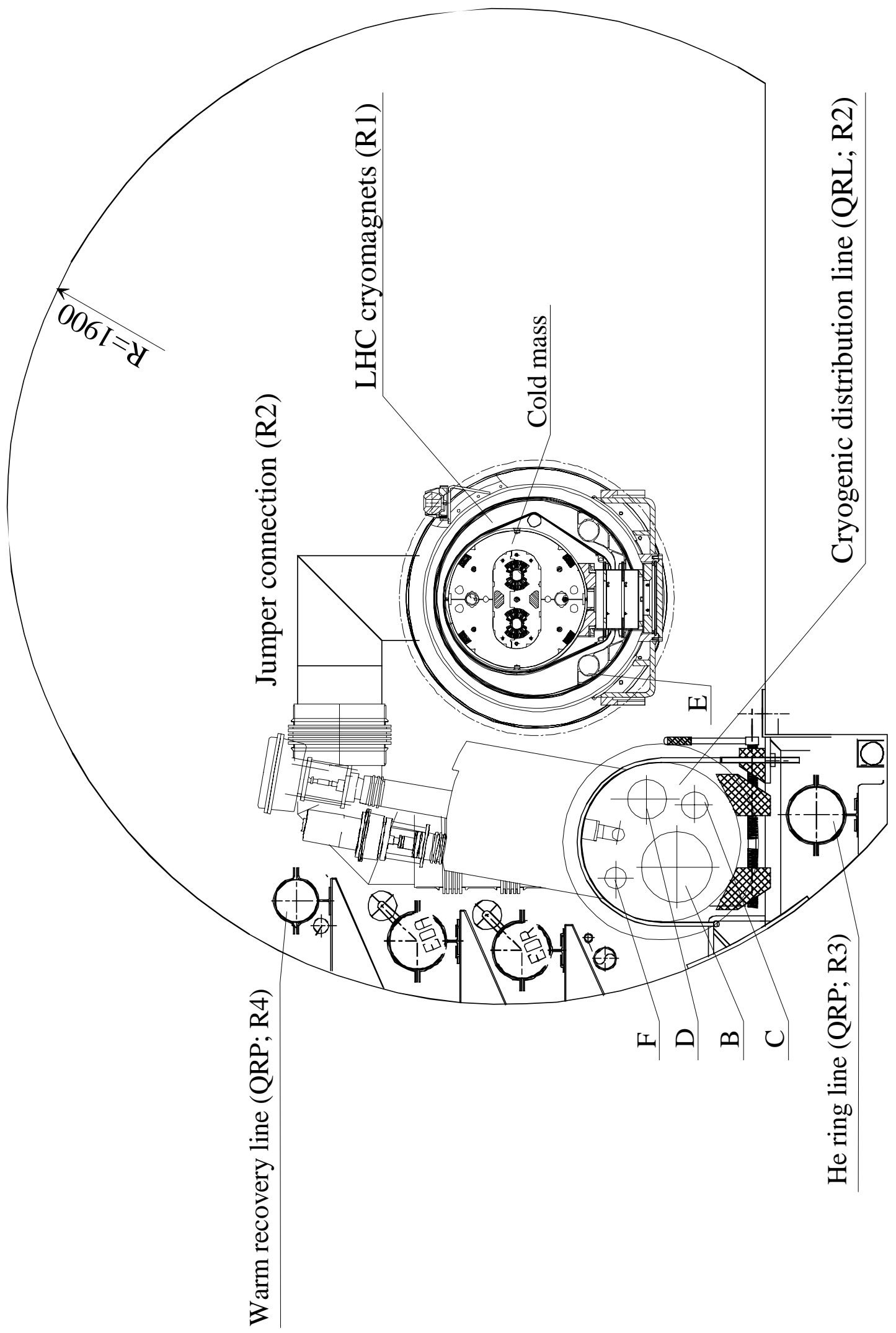

Figure 8: LHC machine components located in the tunnel. 

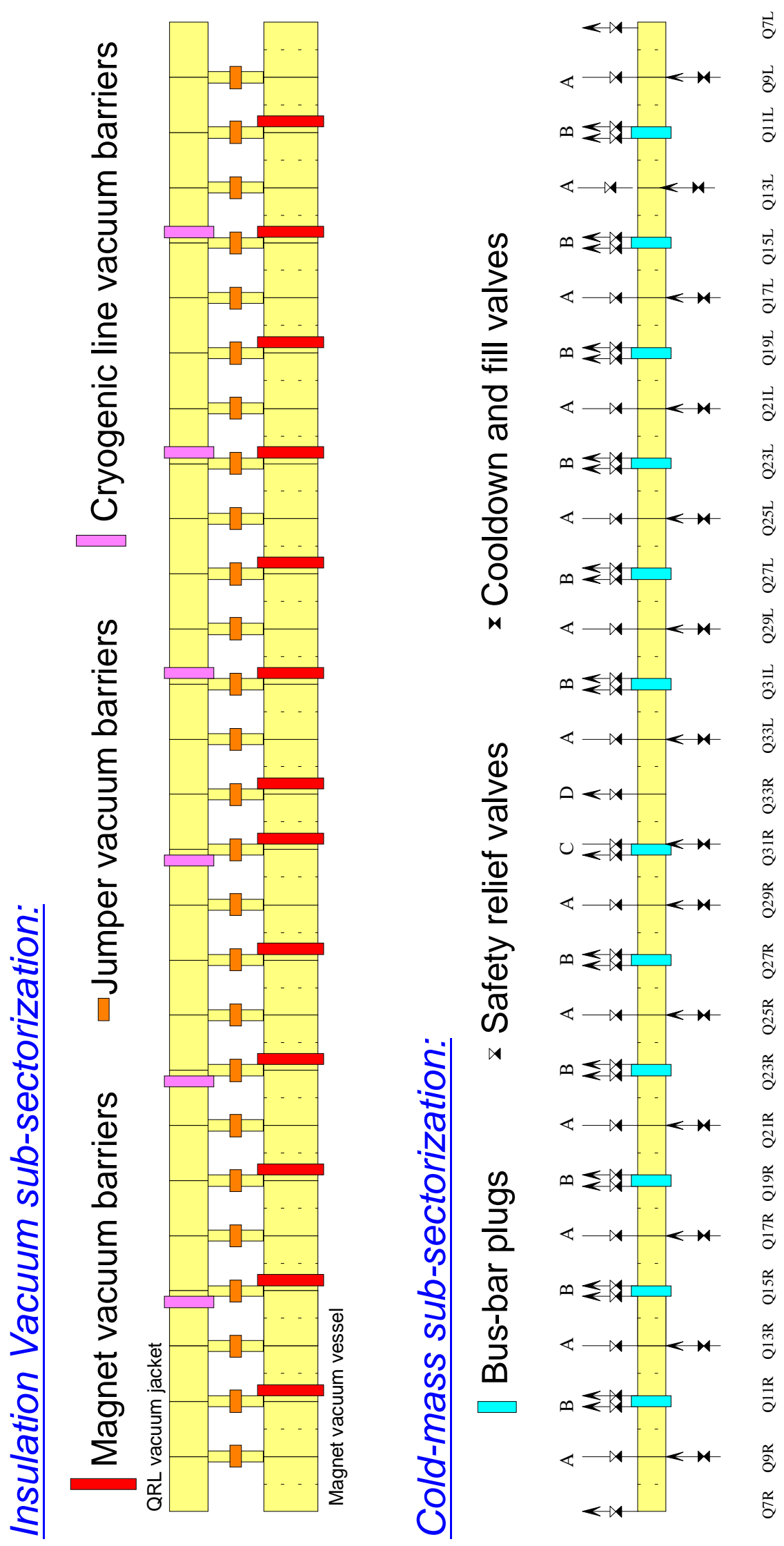

Figure 9: Vacuum and cold mass sub-sectorization (QRL and LHC machine cryostat) in the $L H C$ regular arc 


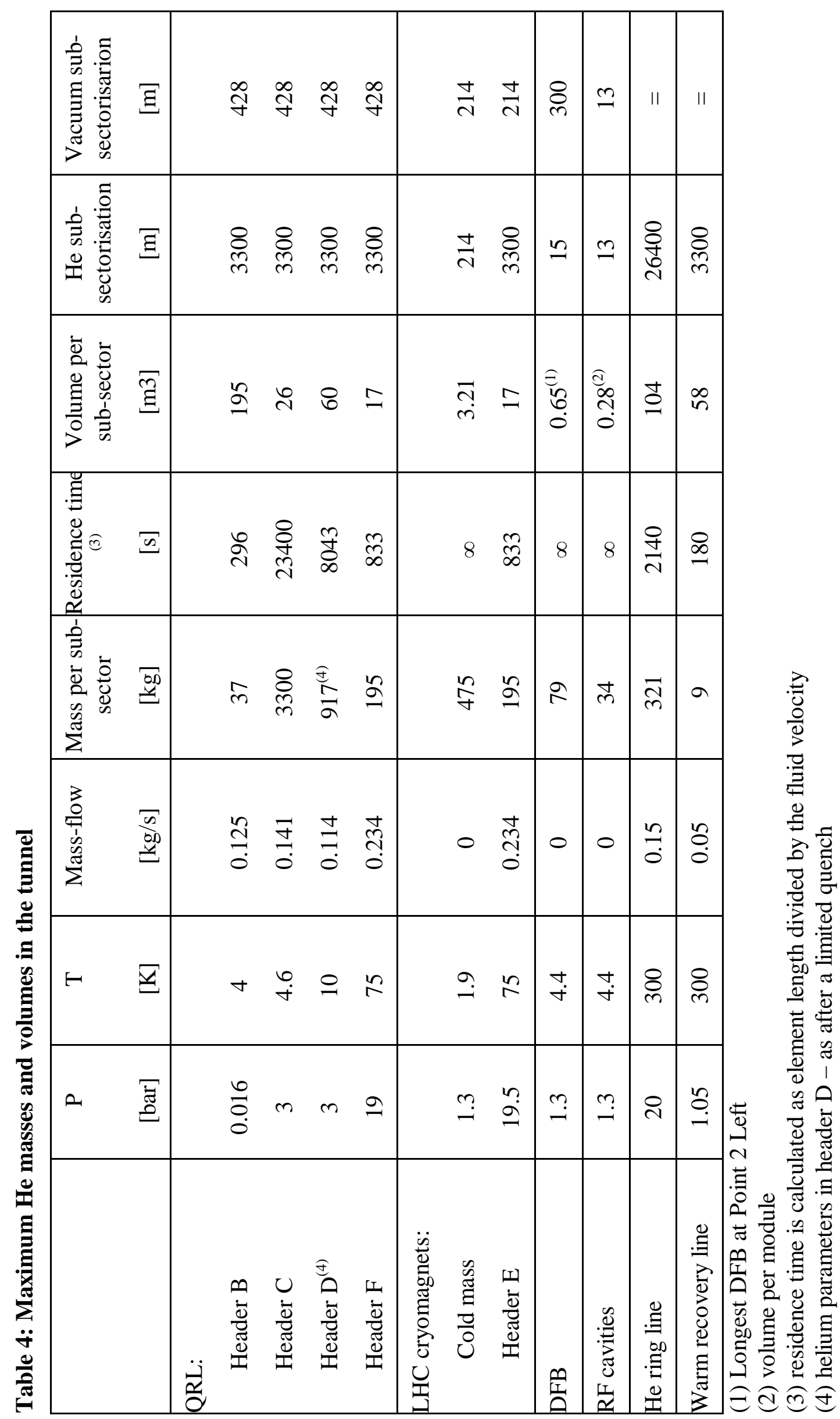




\subsection{Potential and credible failures at the nodes located in the tunnel}

All potential failures at the cryogenic nodes located in the tunnel, which may happen at any phase of the collider operation, are listed in Table 5.

We identify a failure at a node by the following code:

\section{Rx.y}

The first identification character [R], as shown in Figure 2, defines a node location [4].

The second identification character [x], as listed in Table 3, defines a node.

The third identification character [y], as listed in Table 1, defines a failure mode.

In Table 5 all failures listed in Table 1 for every node located in the tunnel are combined with the machine operation modes given in Table 2, creating a full list of potential failures. Subsequently for each potential failure a YES/NO decision is made to eliminate events that are not credible. Finally some of the credible failures are qualified as worst case scenarios on the criterion of mass of helium involved and access to the tunnel as shown in darker cells in Table 5. 


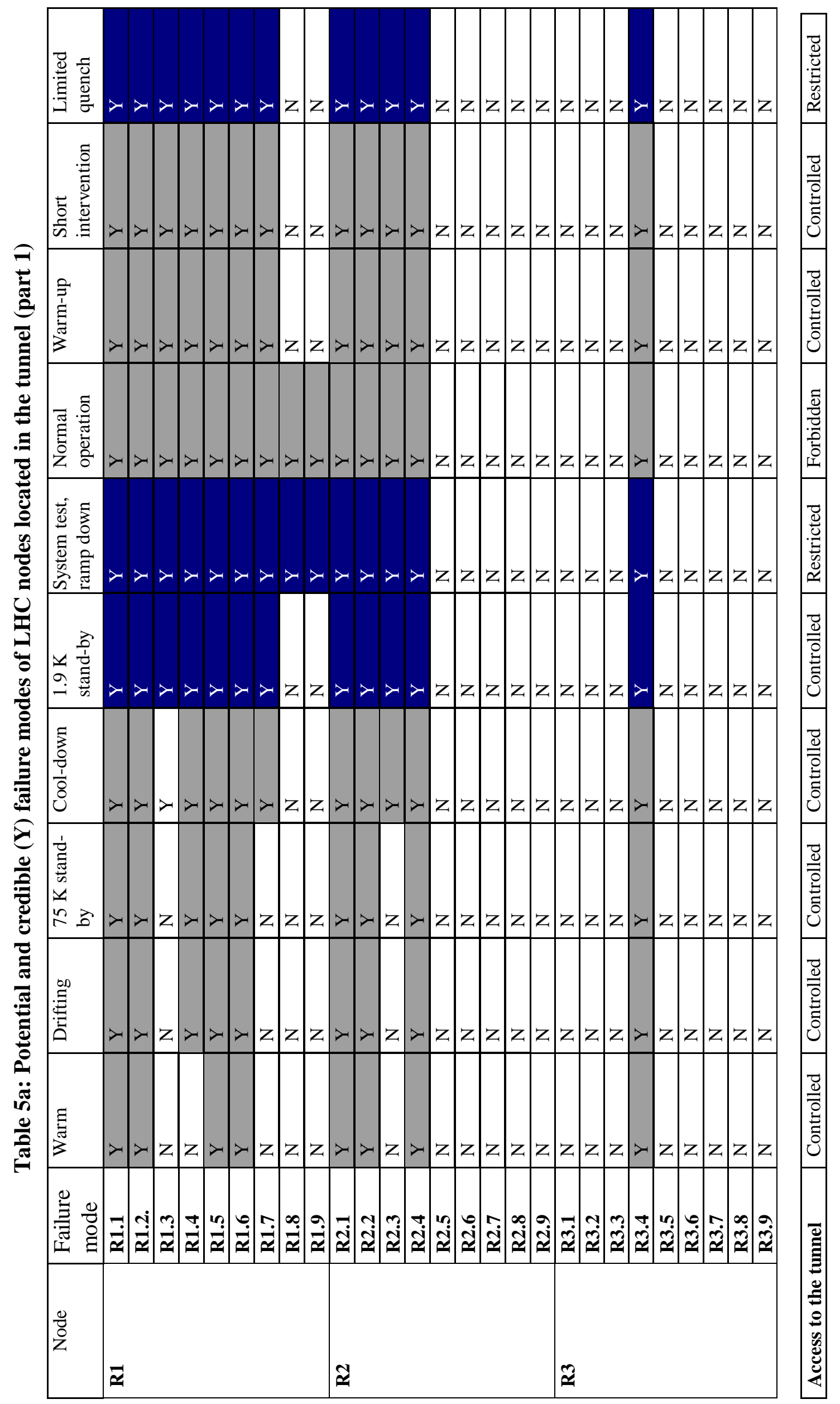




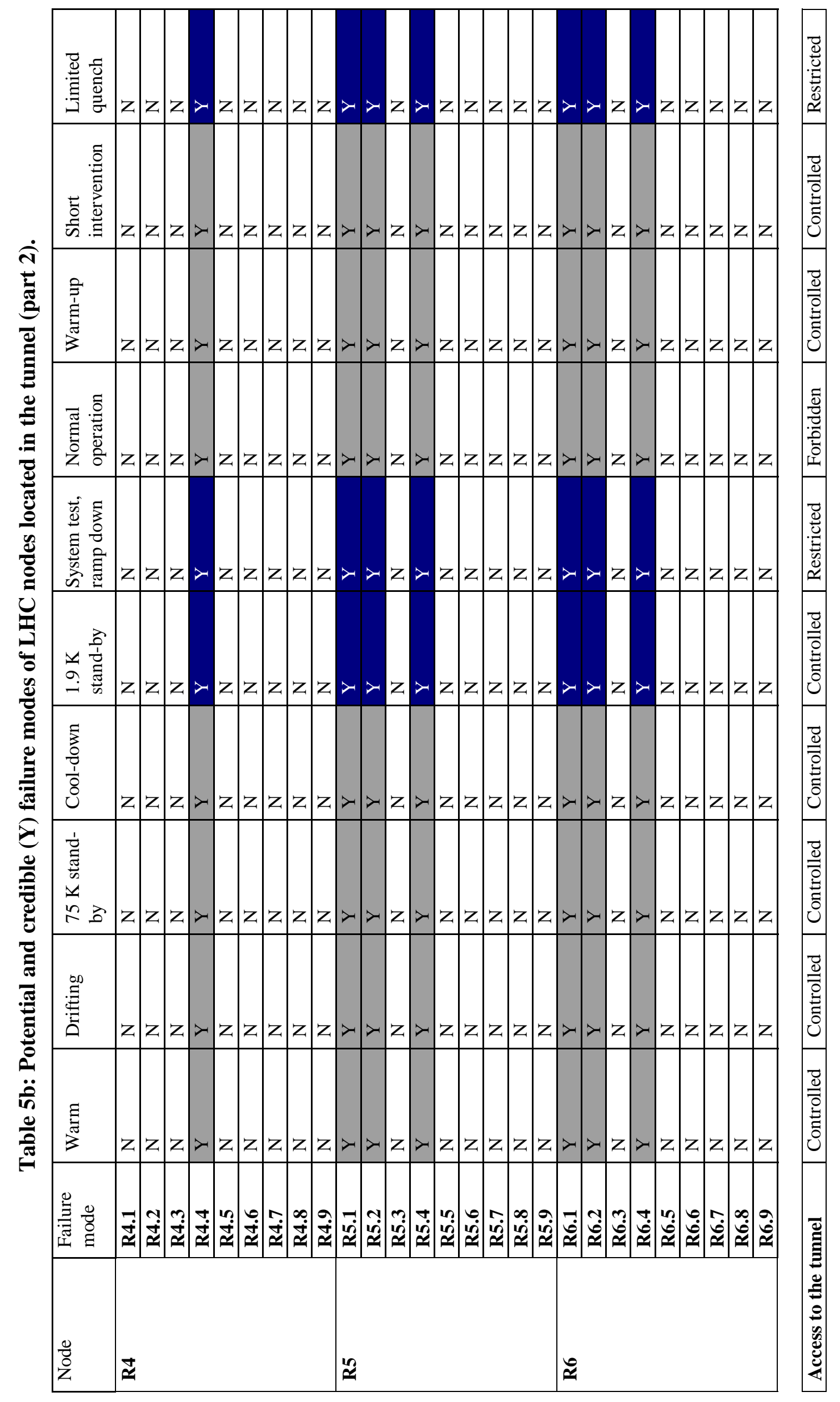




\subsection{Analysis of and recommendations for credible failure modes at the nodes located in the tunnel}

The detailed analysis is performed and recommendations are formulated for the failures involving the highest helium amounts during the operation modes with access to the tunnel allowed or restricted. In Table 6 the credible cryogenics-related failures are gathered and their potential causes are specified. In Table 7 physical consequences of the credible failures are described, the method of failure detection is proposed (column Information/Detection), as well as preventive and corrective defence measures.

Table 6: Credible failures of the cryogenic elements located in the tunnel and their causes

\begin{tabular}{|c|c|c|}
\hline Element & Failure & Failure causes \\
\hline \multirow[t]{9}{*}{ R1. LHC machine cryostat } & R1.1. Air flow to cryostat insulation vacuum & $\begin{array}{l}\text { Mechanical impact on a vacuum tapping } \\
\text { Bellows failure } \\
\text { Weld non-tight }\end{array}$ \\
\hline & $\begin{array}{l}\text { R1.2. Helium flow to cryostat insulation } \\
\text { vacuum }\end{array}$ & $\begin{array}{l}\text { Bellows failure } \\
\text { Weld non-tight }\end{array}$ \\
\hline & $\begin{array}{l}\text { R1.3. Air flow to sub-atmospheric helium } \\
\text { Venting to helium of cryostat insulation } \\
\text { vacuum }\end{array}$ & Break of instrumentation capillary \\
\hline & R1.4. Helium flow to environment & Break of instrumentation capillary \\
\hline & R1.5. Air flow to beam vacuum & $\begin{array}{l}\text { Break of warm beam tube e.g. due to } \\
\text { beam escape. } \\
\text { Bellows failure }\end{array}$ \\
\hline & R1.6. Helium flow to beam vacuum & $\begin{array}{l}\text { Break of beam screen cooling pipe or } \\
\text { cold beam tube e.g. due to beam escape. }\end{array}$ \\
\hline & $\begin{array}{l}\text { R1.7. Pressurized helium flow to sub- } \\
\text { atmospheric helium }\end{array}$ & Bellows failure \\
\hline & R1.8. Energy release due to a sector quench & $\begin{array}{l}\text { Control system failure } \\
\text { Utilities failure }\end{array}$ \\
\hline & R1.9 Energy release due to electrical arc & Low dielectric resistance of helium \\
\hline \multirow[t]{4}{*}{$\begin{array}{l}\text { R2. Cryogenic distribution line } \\
\text { QRL with jumper connection }\end{array}$} & R2.1. Air flow to QRL insulation vacuum & $\begin{array}{l}\text { Mechanical impact on a vacuum gauge } \\
\text { Bellows failure } \\
\text { Weld non-tight }\end{array}$ \\
\hline & R2.2. Helium flow to QRL insulation vacuum & $\begin{array}{l}\text { Bellows failure } \\
\text { Weld non-tight }\end{array}$ \\
\hline & R2.3 Air flow to sub-atmospheric helium & Break of helium guard \\
\hline & R2.4. Helium flow to environment & $\begin{array}{l}\text { Break of jumper connection due to } \\
\text { mechanical impact or geotechnical } \\
\text { problems }\end{array}$ \\
\hline R3. Helium ring line & R3.4. Helium flow to environment & Mechanical impact on helium ring line \\
\hline R4. Warm helium recovery line & R4.1. Helium flow to environment & $\begin{array}{l}\text { Mechanical impact on warm helium } \\
\text { recovery line }\end{array}$ \\
\hline \multirow[t]{3}{*}{ R5. Electrical feed box DFB } & R5.1. Air flow to DFB insulation vacuum & Mechanical impact on a vacuum gauge \\
\hline & R5.2. Helium flow to DFB insulation vacuum & $\begin{array}{l}\text { Bellows failure } \\
\text { Weld non-tight }\end{array}$ \\
\hline & R5.4 Helium flow to environment & Brake of instrumentation capillary \\
\hline \multirow[t]{3}{*}{ R6. RF cavities } & $\begin{array}{l}\text { R6.1. Air flow to RF cavities insulation } \\
\text { vacuum }\end{array}$ & $\begin{array}{l}\text { Mechanical impact on a vacuum gauge } \\
\text { Bellows failure } \\
\text { Weld non-tight }\end{array}$ \\
\hline & R6.2. Helium flow to RF insulation vacuum & $\begin{array}{l}\text { Bellows failure } \\
\text { Weld non-tight }\end{array}$ \\
\hline & R6.4 Helium flow to environment & Brake of instrumentation capillary \\
\hline
\end{tabular}




\begin{tabular}{|c|c|c|c|c|}
\hline & 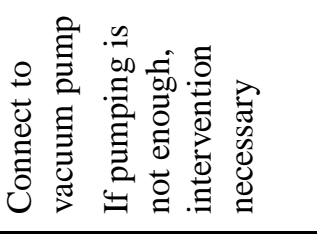 & 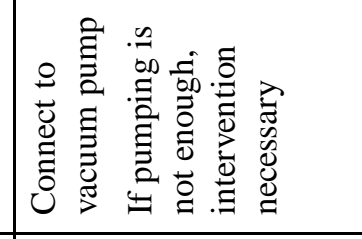 & 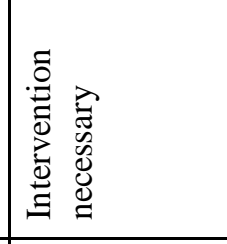 & 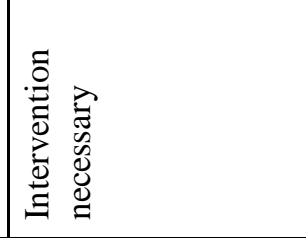 \\
\hline & 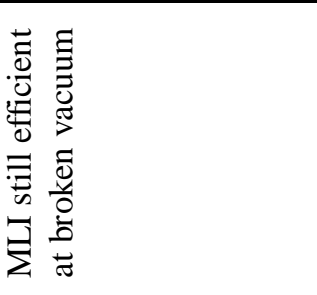 & 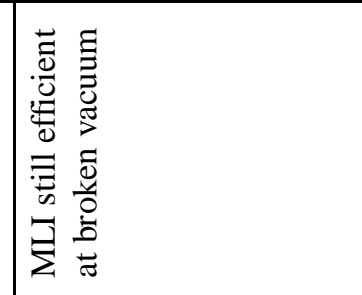 & 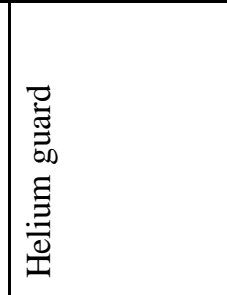 & 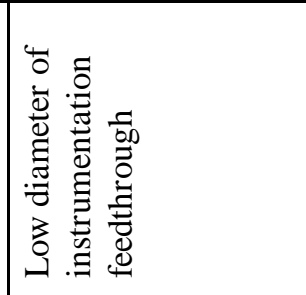 \\
\hline$\frac{\partial}{\partial}$ & 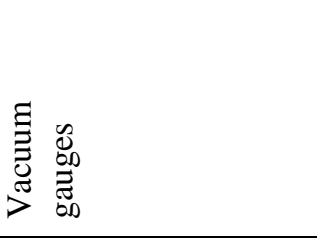 & 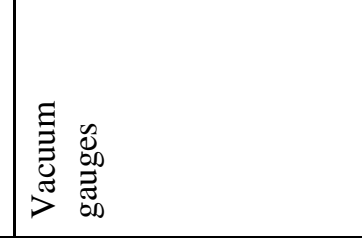 & 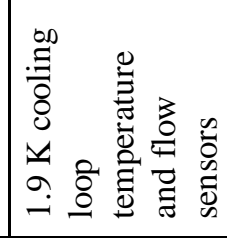 & 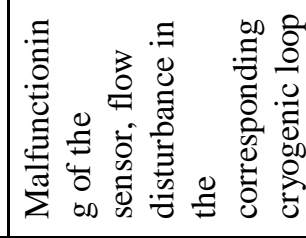 \\
\hline & 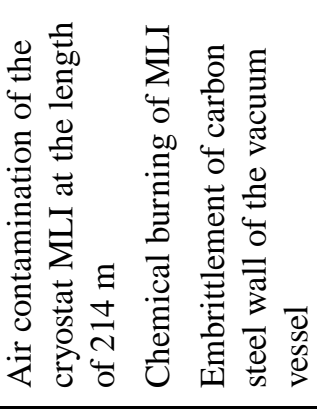 & 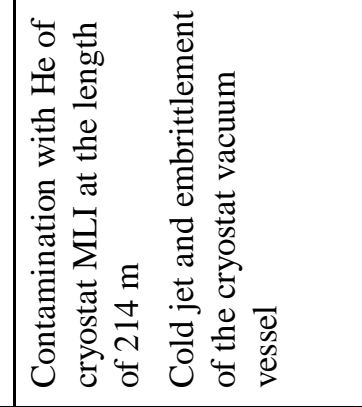 & 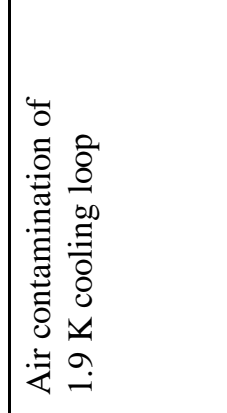 & 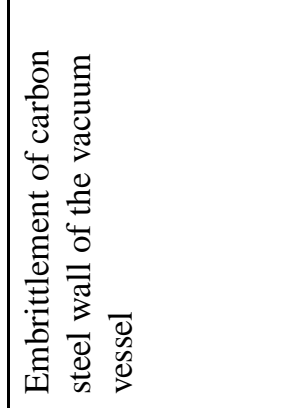 \\
\hline & $\check{z}$ & 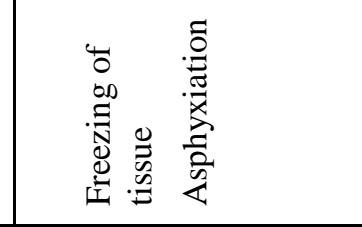 & ஜ & 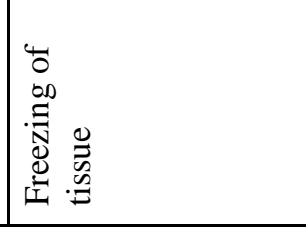 \\
\hline & 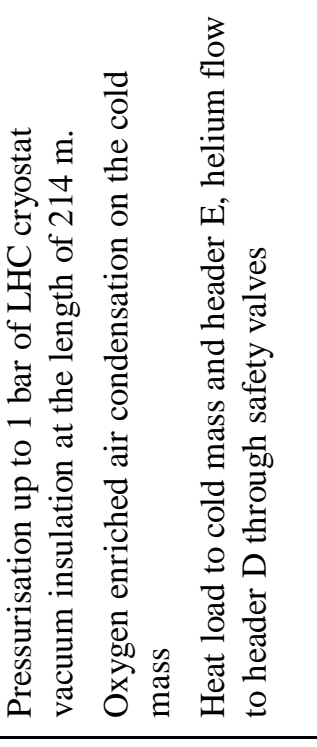 & 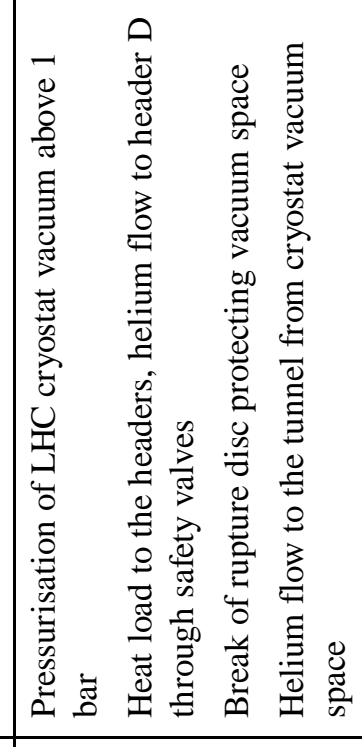 & 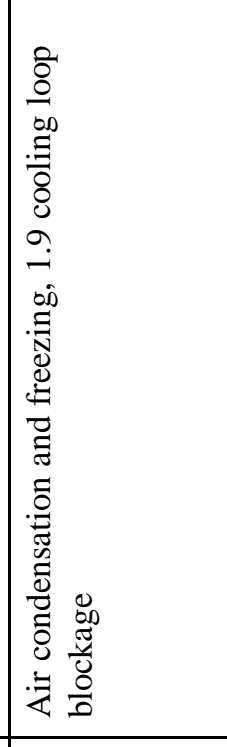 & 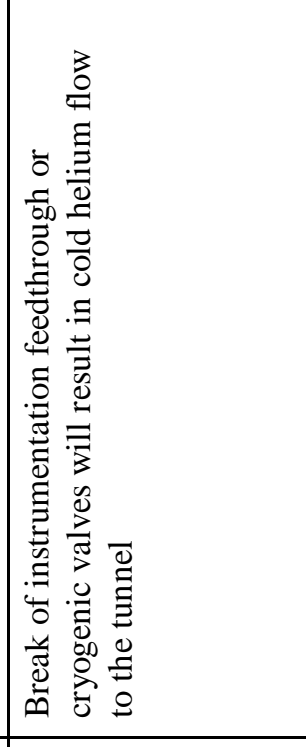 \\
\hline & 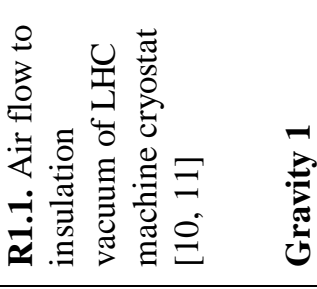 & 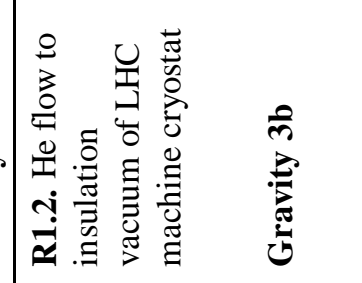 & 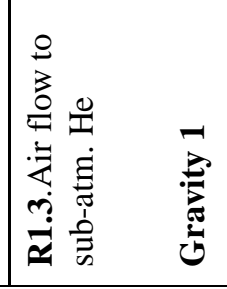 & 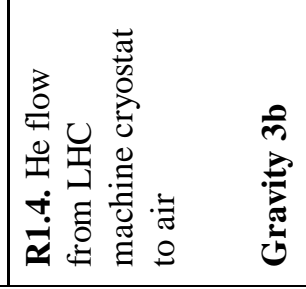 \\
\hline
\end{tabular}




\begin{tabular}{|c|c|c|c|c|c|c|}
\hline 这 & 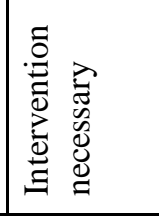 & 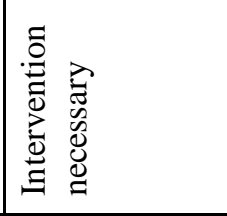 & 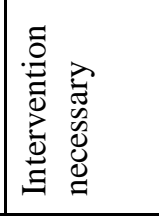 & 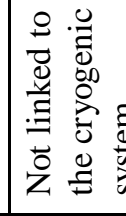 & & 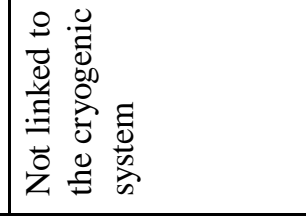 \\
\hline 若 & 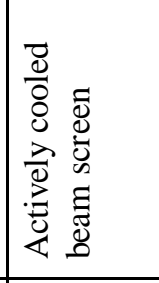 & 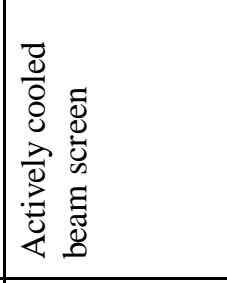 & 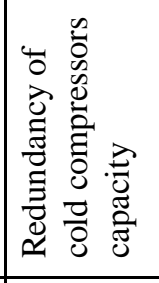 & 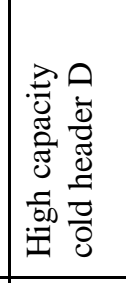 & & 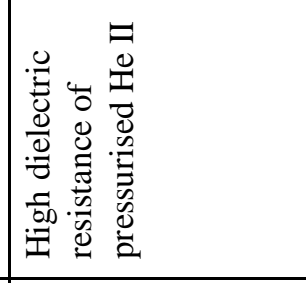 \\
\hline 害 & 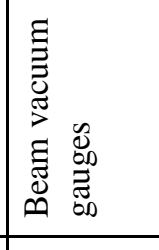 & 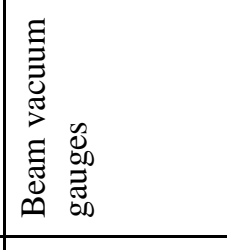 & 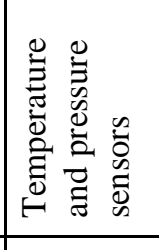 & 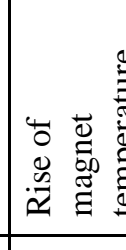 & & 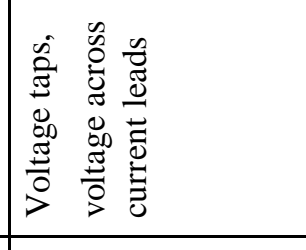 \\
\hline 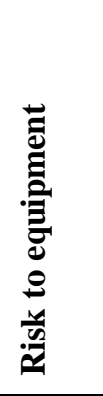 & 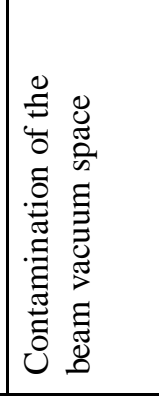 & 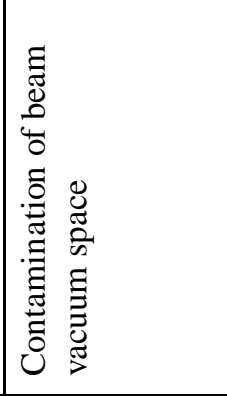 & $\check{z}$ & & $\frac{8}{8}$ & $\mid \begin{array}{l}\tilde{\vec{\nu}} \\
\bar{\nu} \\
\tilde{w}\end{array}$ \\
\hline 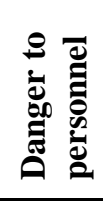 & ż & 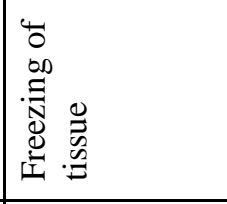 & 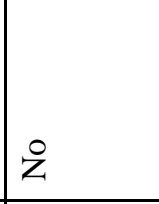 & & 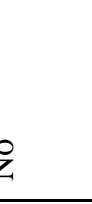 & 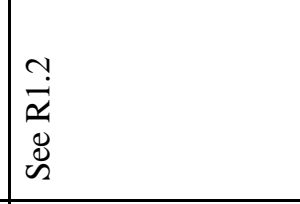 \\
\hline 咅 & 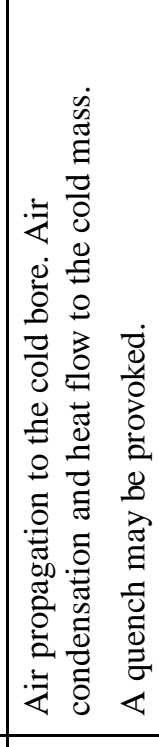 & 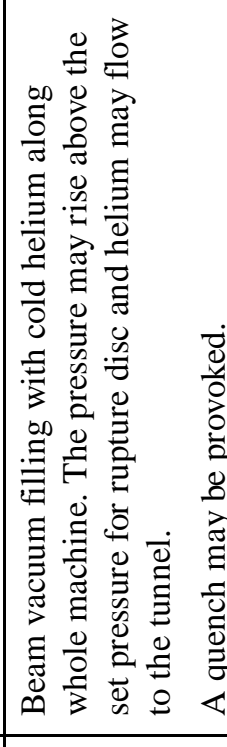 & 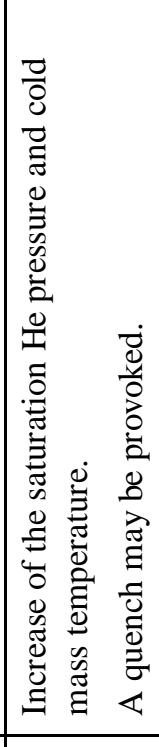 & 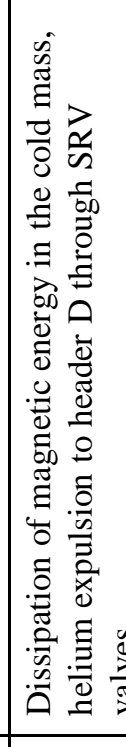 & & 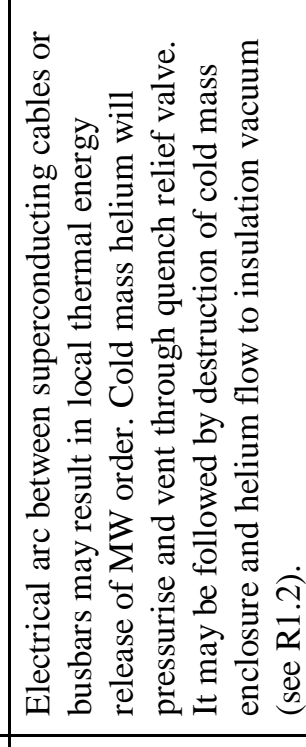 \\
\hline 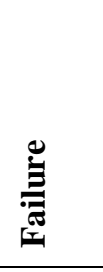 & 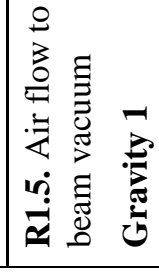 & 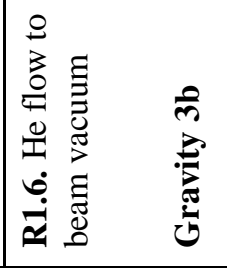 & 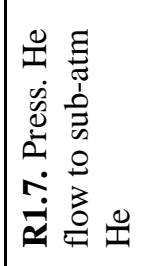 & 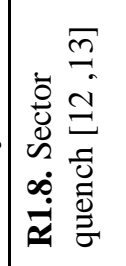 & & 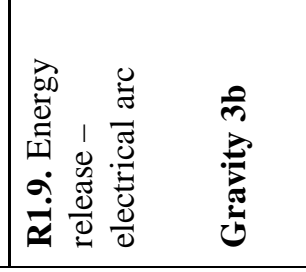 \\
\hline
\end{tabular}




\begin{tabular}{|c|c|c|c|c|}
\hline 造 & 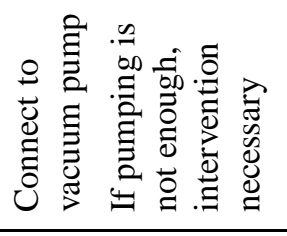 & 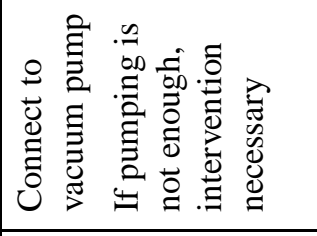 & 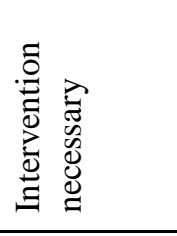 & 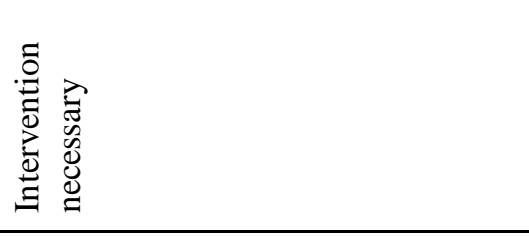 \\
\hline 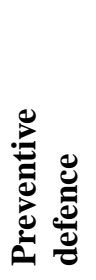 & 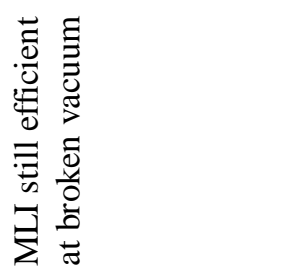 & 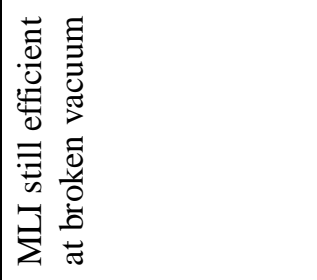 & 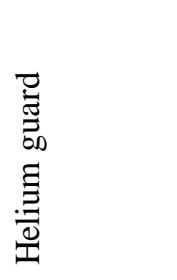 & 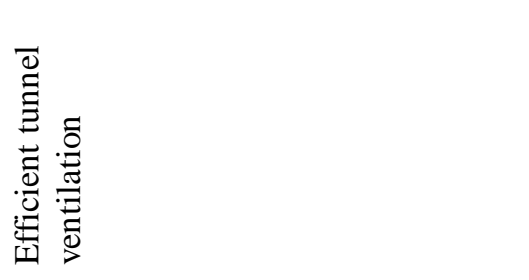 \\
\hline 施 & 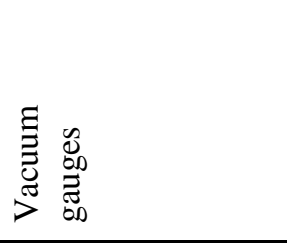 & 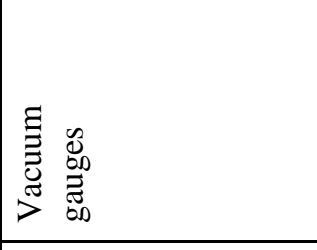 & 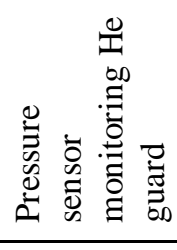 & 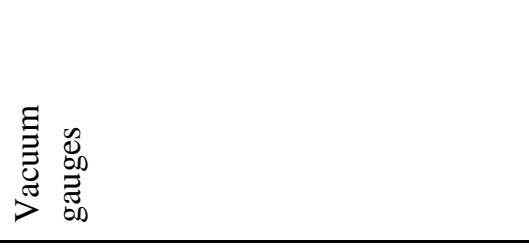 \\
\hline 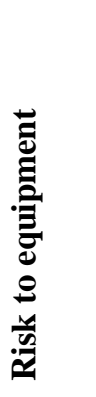 & 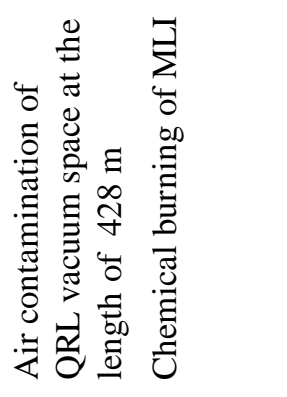 & 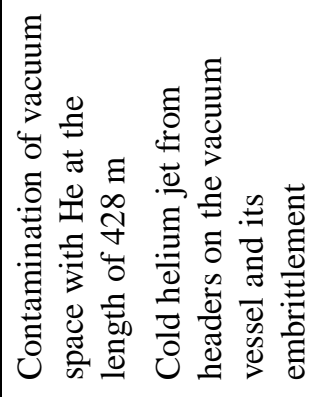 & 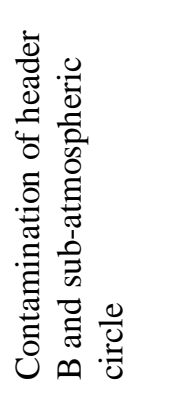 & 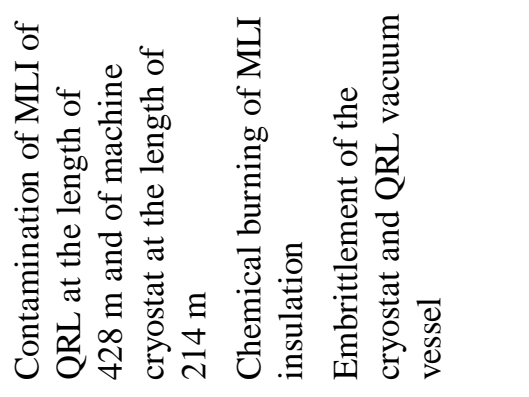 \\
\hline 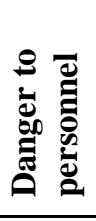 & z & 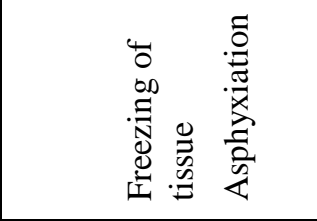 & z & 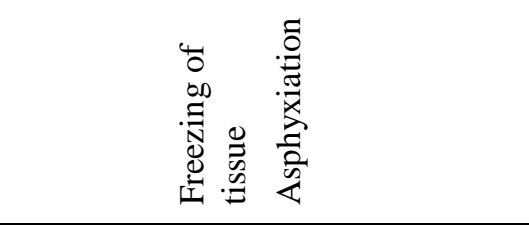 \\
\hline 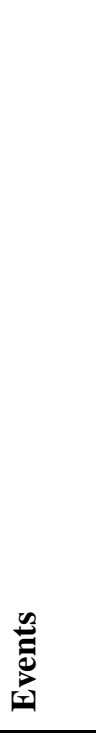 & 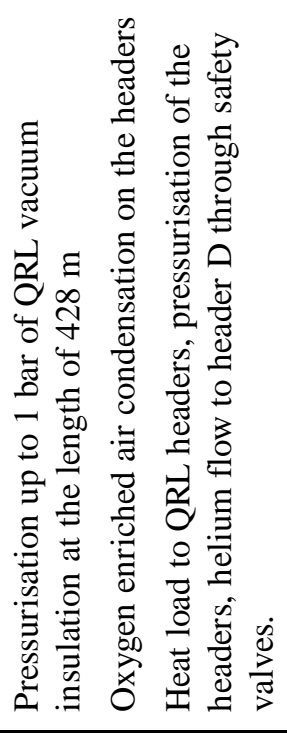 & 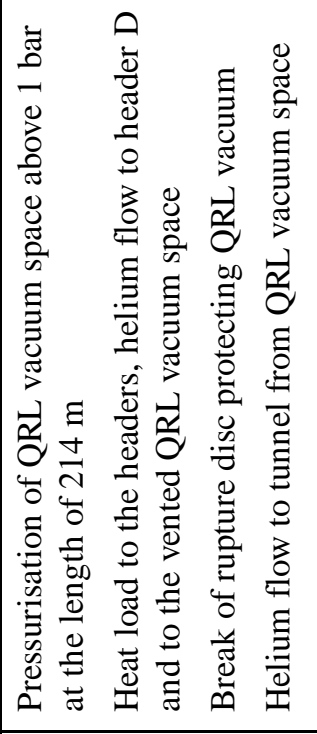 & 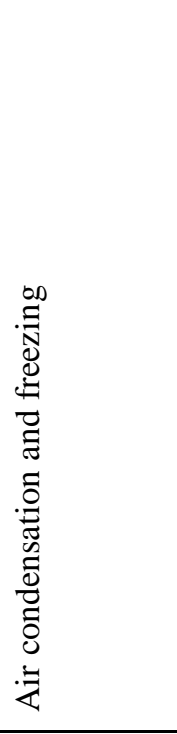 & 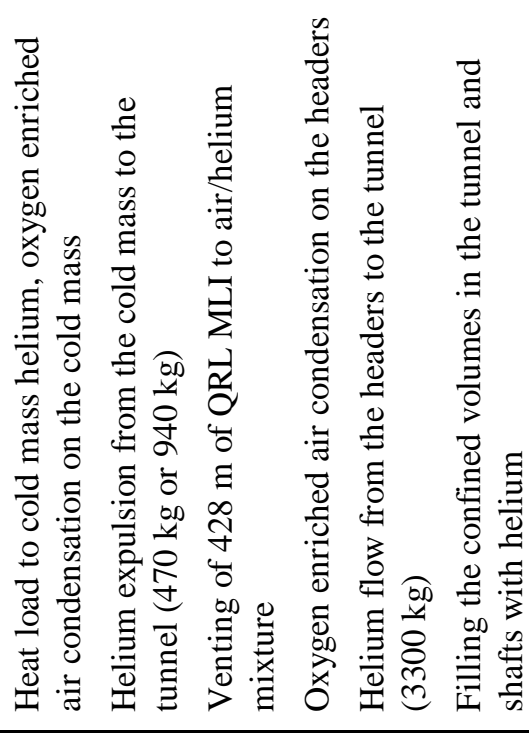 \\
\hline 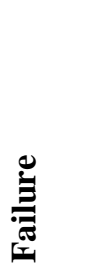 & 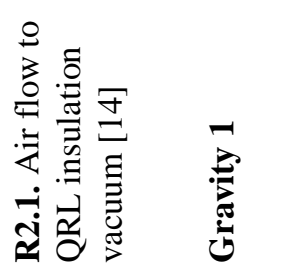 & 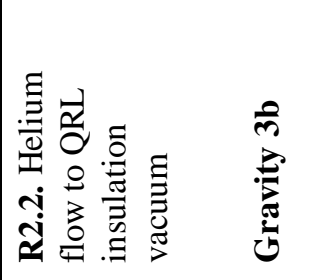 & 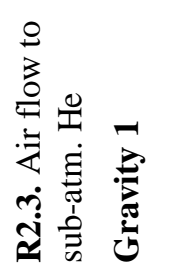 & 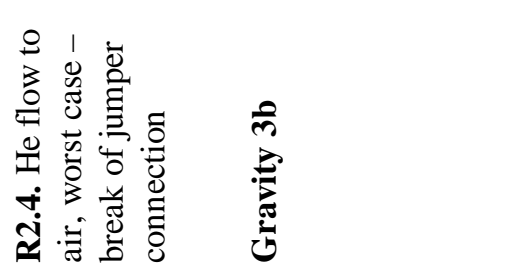 \\
\hline
\end{tabular}




\begin{tabular}{|c|c|c|c|c|}
\hline لْ & 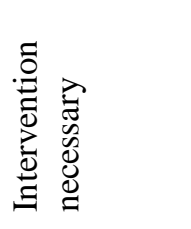 & 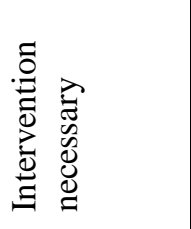 & 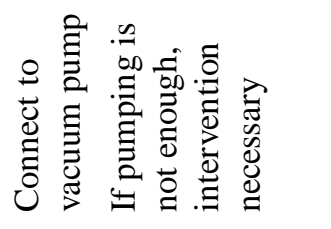 & 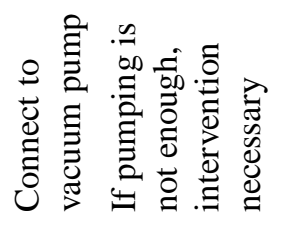 \\
\hline 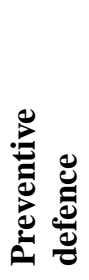 & 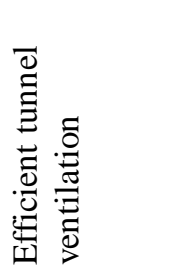 & 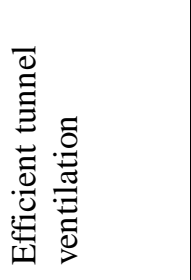 & 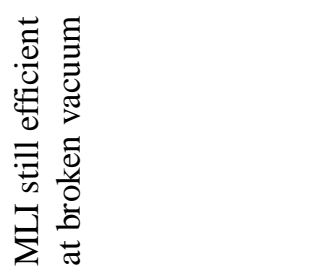 & 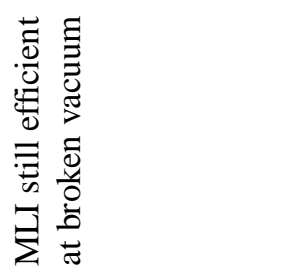 \\
\hline 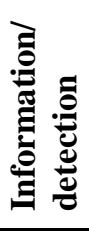 & 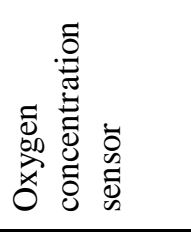 & 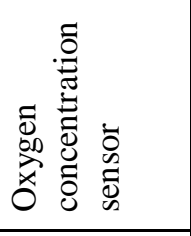 & 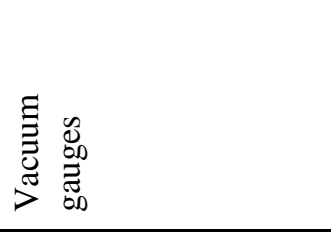 & 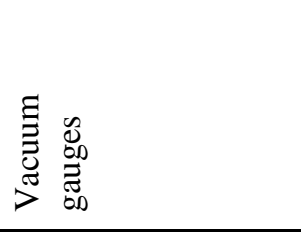 \\
\hline 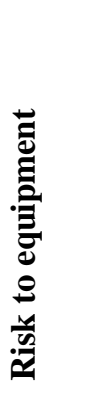 & ż & $\stackrel{\circ}{z}$ & 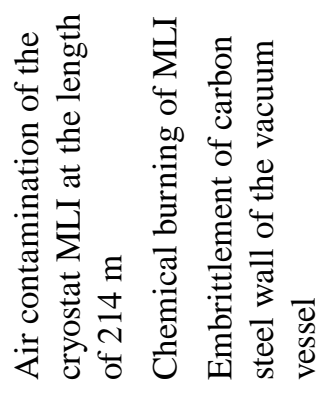 & 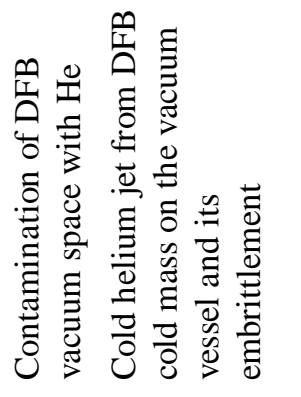 \\
\hline 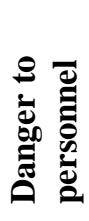 & 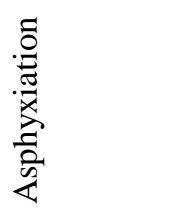 & 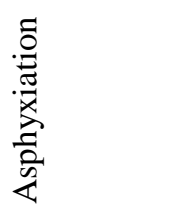 & $\stackrel{\circ}{z}$ & 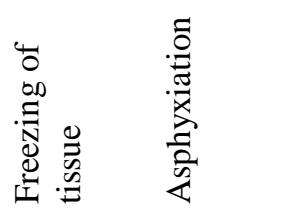 \\
\hline 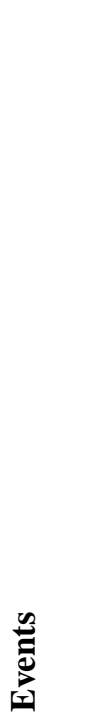 & 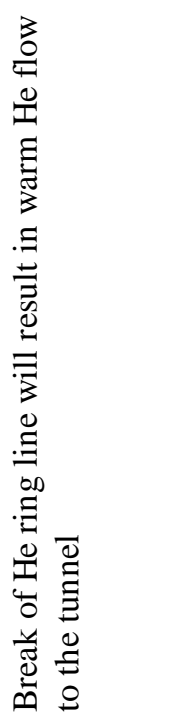 & 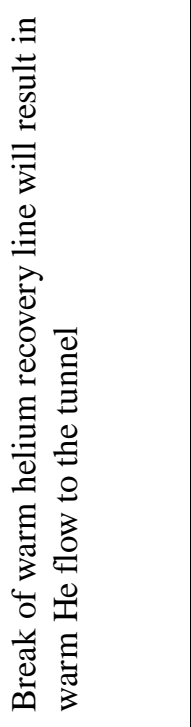 & 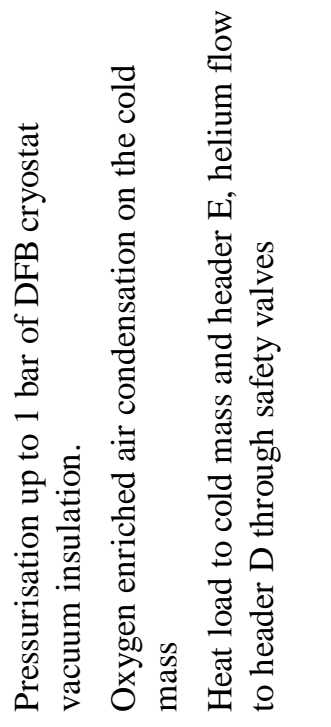 & 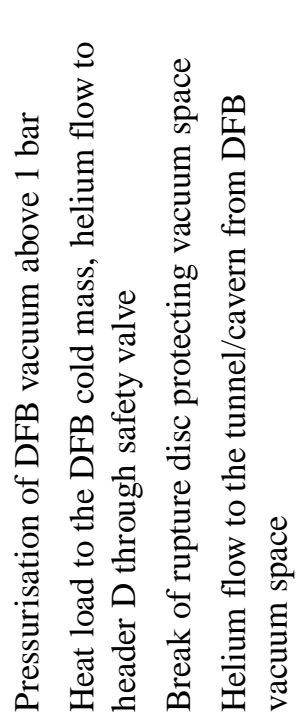 \\
\hline 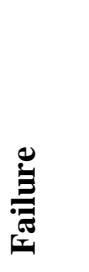 & 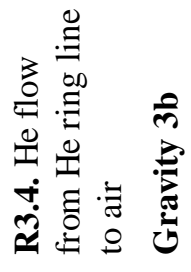 & 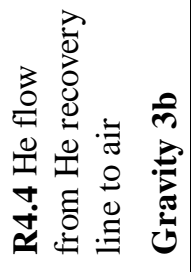 & 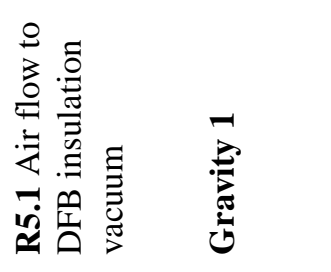 & 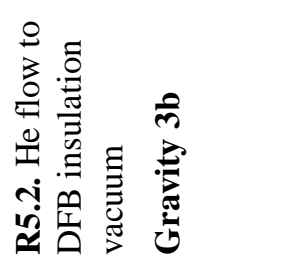 \\
\hline
\end{tabular}




\begin{tabular}{|c|c|c|c|c|}
\hline 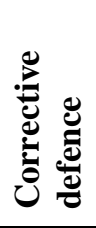 & 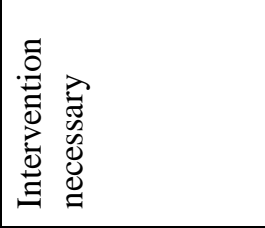 & 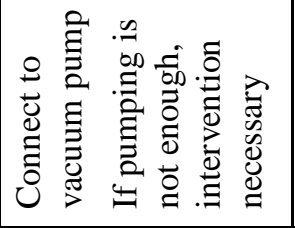 & 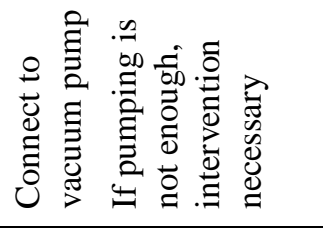 & 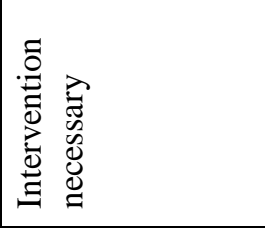 \\
\hline 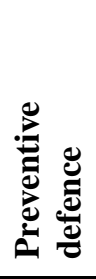 & 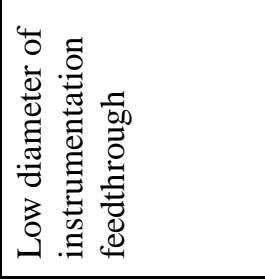 & 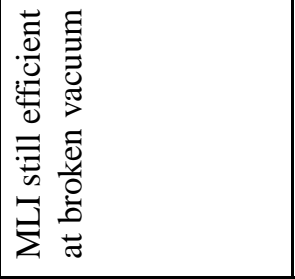 & 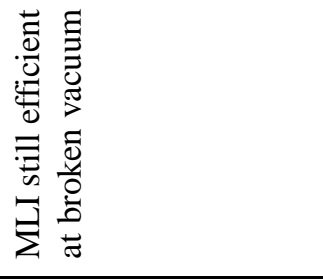 & 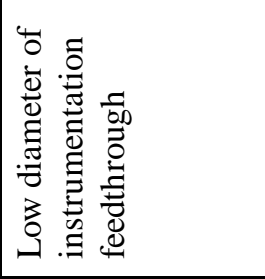 \\
\hline 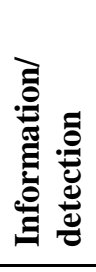 & 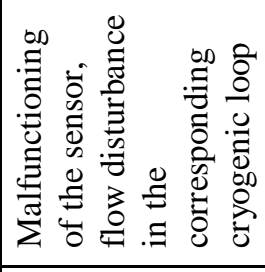 & 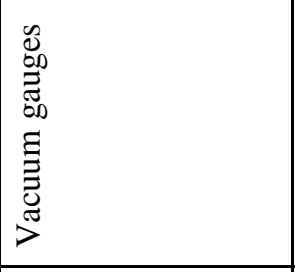 & 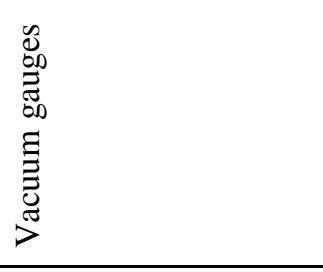 & 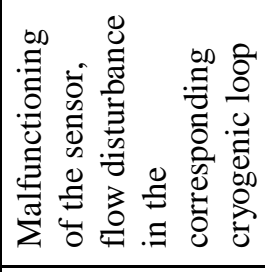 \\
\hline 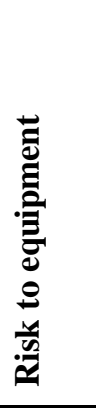 & 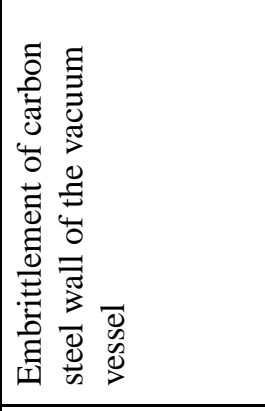 & 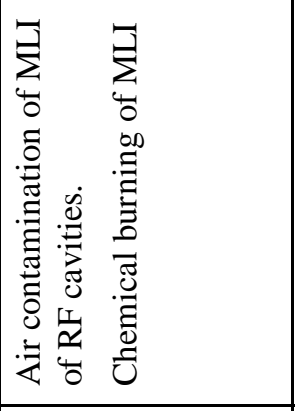 & 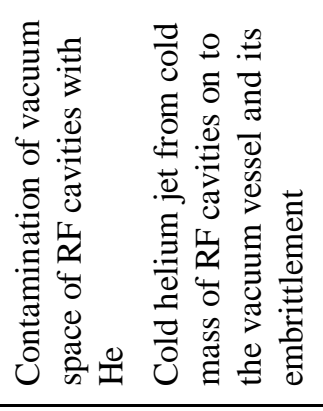 & 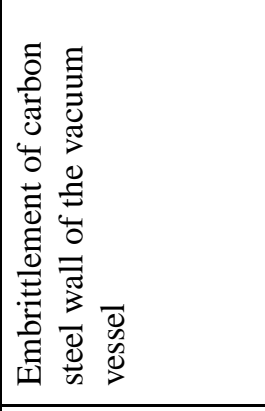 \\
\hline 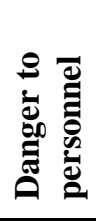 & 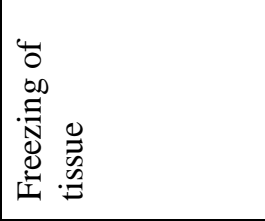 & $\stackrel{\circ}{z}$ & 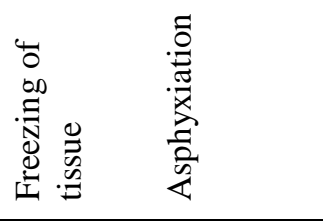 & 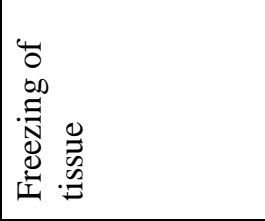 \\
\hline 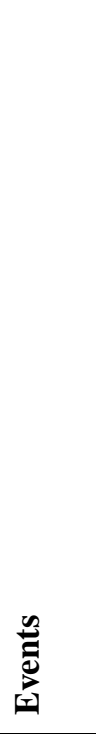 & 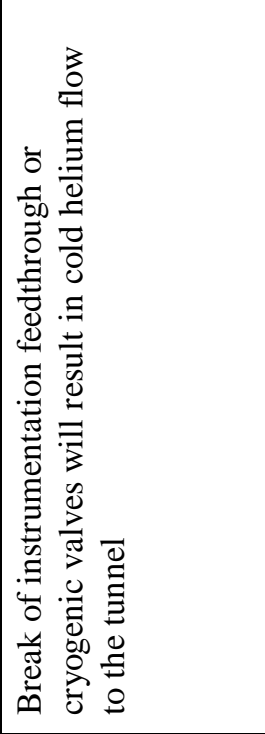 & 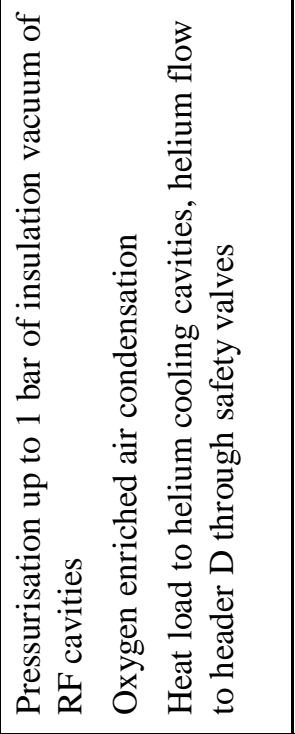 & 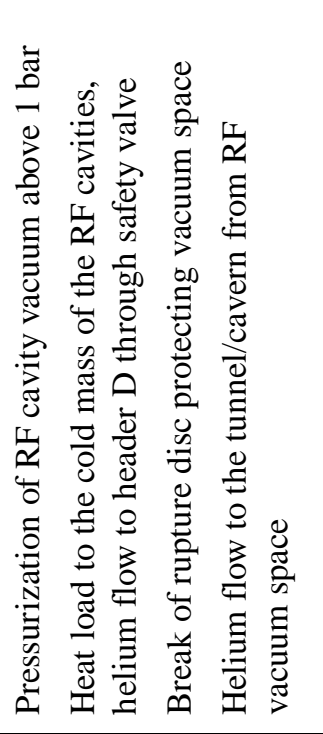 & 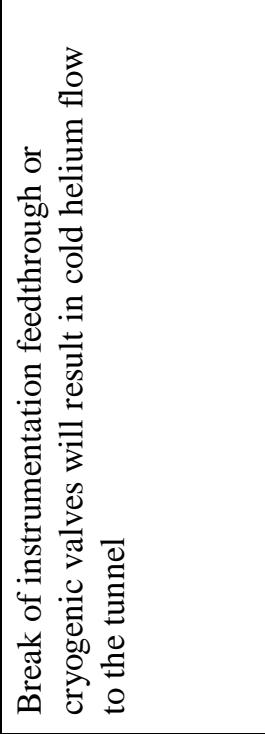 \\
\hline 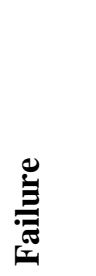 & 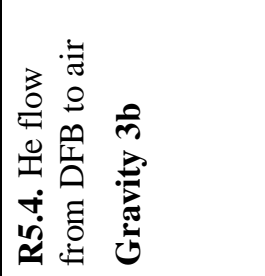 & 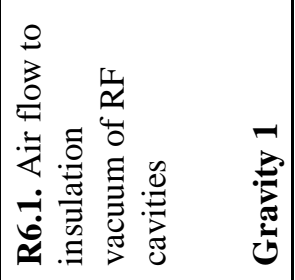 & 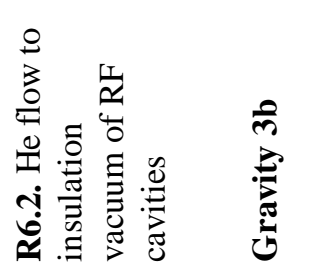 & 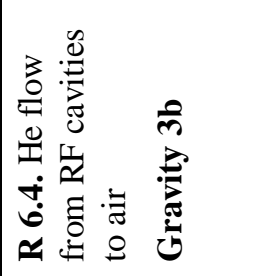 \\
\hline
\end{tabular}


There are 12 events of the gravity $3 \mathrm{~b}$ identified in Table 7 . Table 8 lists the $3 \mathrm{~b}$ gravity events, gives the highest amounts of helium that might be relieved to the tunnel together with approximate mass-flow rates and formulates the recommendations. Generally the highest value of the mass-flow rate occurs in a period of about 100 seconds. After reaching this value the mass-flow rate reduces at least by an order of magnitude within a similar period of time.

Table 8: Gravity $3 b$ failures at the nodes located in the tunnel

\begin{tabular}{|c|c|c|}
\hline Failure & $\begin{array}{l}\text { Maximum amount of helium } \\
\text { relieved to the tunnel }[\mathrm{kg}] / \\
\text { approximate flow rate }[\mathrm{kg} / \mathrm{s}]\end{array}$ & Recommendations \\
\hline $\begin{array}{l}\text { R1.2 LHC cryomagnets: He flow to } \\
\text { cryostat insulation vacuum }\end{array}$ & 475 / below 2 & Further analysis necessary \\
\hline $\begin{array}{l}\text { R1.4 LHC cryomagnets: He flow to } \\
\text { air }\end{array}$ & 475 / He flow of a leak order & $\begin{array}{l}\text { Consequences of the failure fully } \\
\text { covered by } \mathrm{R} 1.2 \text { and } \mathrm{R} 2.4\end{array}$ \\
\hline $\begin{array}{l}\text { R1.6 LHC cryomagnets He flow to } \\
\text { beam vacuum }\end{array}$ & 475 / to be calculated & Further analysis necessary \\
\hline $\begin{array}{l}\text { R1.9 LHC cryomagnets energy } \\
\text { release-electrical arc }\end{array}$ & $475 \mathrm{~kg}$ / flow rate similar to $\mathrm{R} 1.2$ & $\begin{array}{l}\text { Not directly related to cryogenics, } \\
\text { consequences covered by R } 1.2\end{array}$ \\
\hline $\begin{array}{l}\text { R2.2 He flow to QRL insulation } \\
\text { vacuum, assumption of header C } \\
\text { break }\end{array}$ & $3300 \mathrm{~kg} /$ lower than 2 & $\begin{array}{l}\text { Second, after R2.4 worst case } \\
\text { scenario, further modelling of } \\
\text { helium expulsion to the tunnel as } \\
\text { well as helium propagation in the } \\
\text { tunnel is necessary }\end{array}$ \\
\hline $\begin{array}{l}\text { R2.4 He flow to air, assumption of } \\
\text { jumper connection break }\end{array}$ & $\begin{array}{l}4250 / \text { about } 20 . \\
\text { About } 600 \mathrm{~kg} \text { are discharged in the } \\
\text { first } 60 \mathrm{~s} \text {. After this time the mass } \\
\text { flow-rate reduces by an order of } \\
\text { magnitude. }\end{array}$ & $\begin{array}{l}\text { Worst case scenario, further } \\
\text { modelling of helium expulsion to } \\
\text { the tunnel as well as helium } \\
\text { propagation in the tunnel is } \\
\text { necessary }\end{array}$ \\
\hline $\begin{array}{l}\text { R3.4 He flow to air, He ring line } \\
\text { break }\end{array}$ & $321 \mathrm{~kg} /$ below 7 & Further analysis recommended \\
\hline $\begin{array}{l}\mathbf{R} 4.4 \mathrm{He} \text { flow to air, warm } \mathrm{He} \\
\text { recovery line break }\end{array}$ & $9 \mathrm{~kg} / \mathrm{He}$ flow of a leak order & $\begin{array}{l}\text { Consequences negligible in } \\
\text { comparison with other cases }\end{array}$ \\
\hline $\begin{array}{l}\text { R5.2 He flow to DFB insulation } \\
\text { vacuum }\end{array}$ & 79 kg / below 2 & Further analysis recommended \\
\hline R5.4 DFB He flow to air & $79 \mathrm{~kg} / \mathrm{He}$ flow of a leak order & $\begin{array}{l}\text { Consequences of the failure fully } \\
\text { covered by R5.2 }\end{array}$ \\
\hline $\begin{array}{l}\text { R6.2 He flow to RF cavities } \\
\text { insulation vacuum }\end{array}$ & $34 \mathrm{~kg} /$ below 2 & Further analysis recommended \\
\hline $\begin{array}{l}\text { R6.4 RF cavities cooling He flow } \\
\text { to air }\end{array}$ & $34 \mathrm{~kg} / \mathrm{He}$ flow of a leak order & $\begin{array}{l}\text { Consequences of the failure fully } \\
\text { covered by R6.2 }\end{array}$ \\
\hline
\end{tabular}

As it follows from Table 8, two worst case scenarios can be identified based on the criterion of mass of helium involved and flow rate to the tunnel: helium flow to QRL insulation vacuum (assumption of header $\mathrm{C}$ break) and break of jumper connection. 


\section{Risks associated to failures of LHC cryogenic system nodes in caverns and shafts}

\subsection{LHC cryogenic system nodes located in the caverns and shafts}

The elements of the cryogenic system located in caverns and shafts are listed in Table 9. A layout of a typical cavern is shown in Figure 10.

The interconnecting warm pipes and cryogenic lines are schematically shown in Figure 11. Each pipe or line is identified by its corresponding equipment code and tag numbering.

Table 9: LHC cryogenic system nodes located in caverns and shafts

\begin{tabular}{|l|l|l|}
\hline Node & Description & Remarks \\
\hline U1 & Cold compressor boxes (QURC) & In the caverns \\
\hline U2 & Cryogenic interconnection boxes (QUI) & In the caverns \\
\hline U3 & Underground Lower Cold Boxes (QURA) & In the caverns \\
\hline P4 & $\begin{array}{l}\text { Interconnecting cryogenic lines (QPLA, QPLB, } \\
\text { QULA and QULC) }\end{array}$ & $\begin{array}{l}\text { In the caverns and } \\
\text { shafts }\end{array}$ \\
\hline P5 & $\begin{array}{l}\text { Interconnecting warm pipes (QPP, QPPD and QRP) } \\
\text { In the caverns and } \\
\text { shafts }\end{array}$ \\
\hline
\end{tabular}

The amount of helium and volume of vacuum space involved in case of failure of the elements in the caverns and shafts are given in Tables 2 to $5[15,16,17]$. 


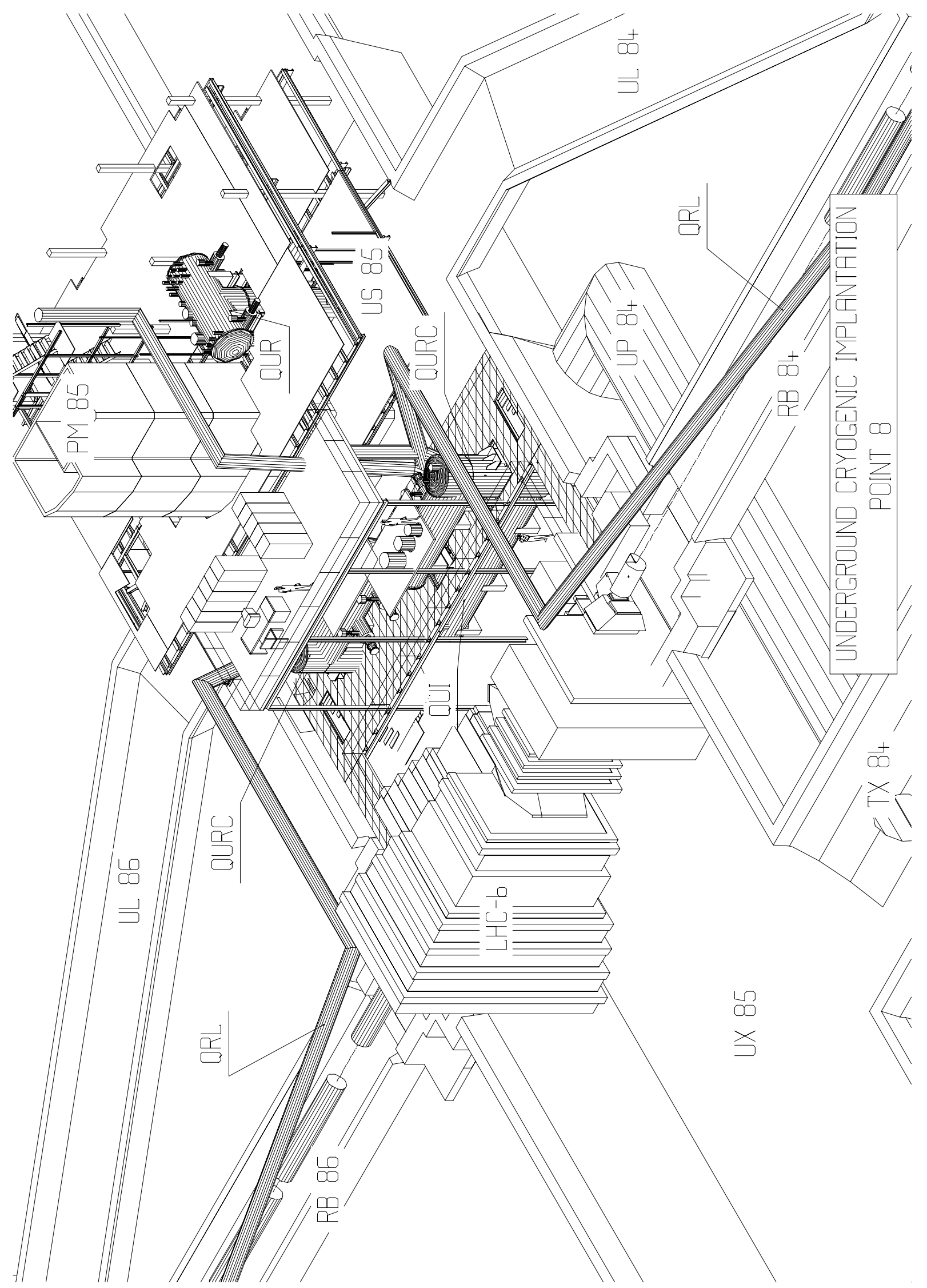

Figure 10: Layout of cryogenic equipment in a typical cavern (P8) 


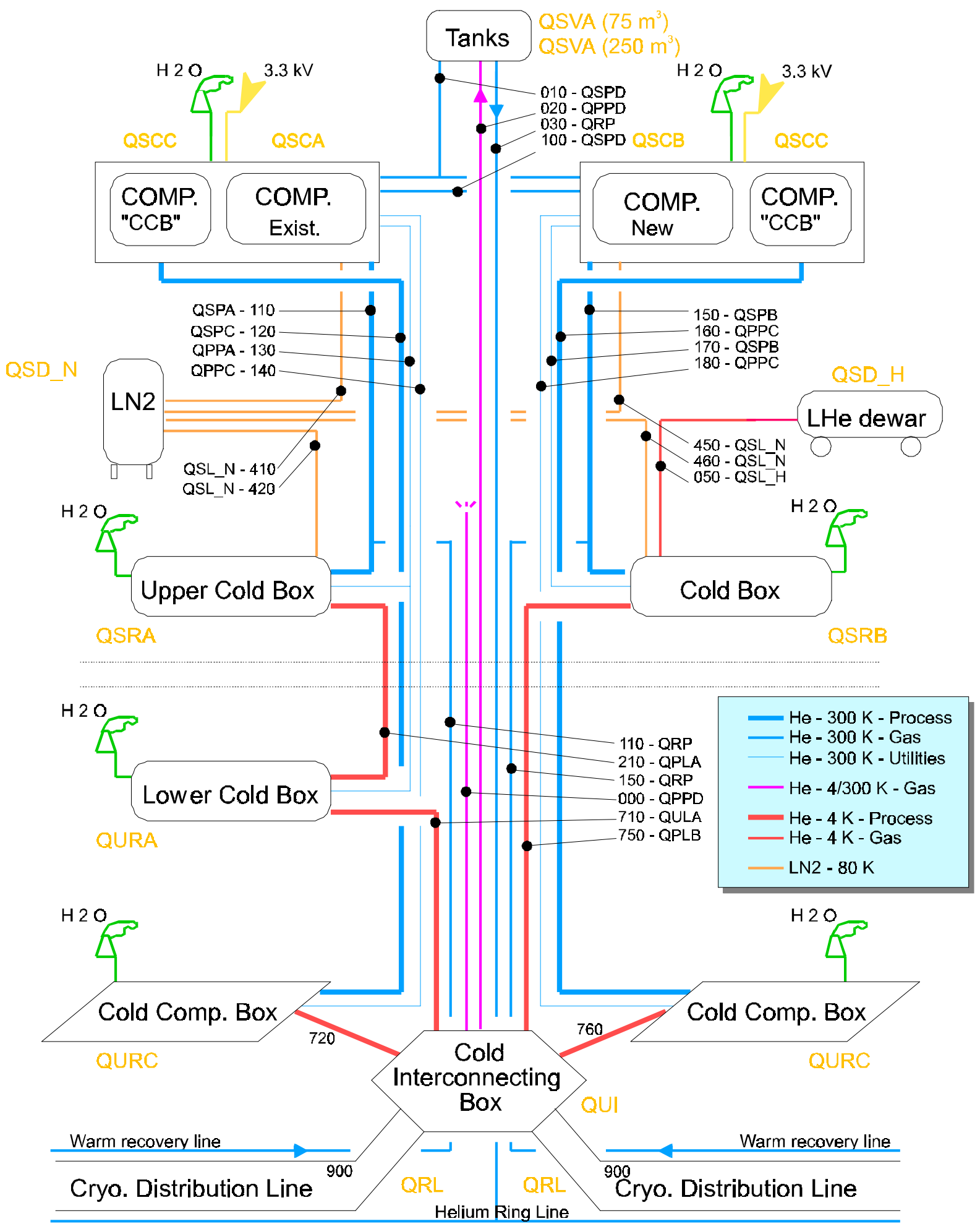

Figure 11: Typical layout of piping at an even-numbered point (P4, P6, P8) 
Table 10: Maximum He masses and volumes in the QURC

\begin{tabular}{|l|c|c|c|c|c|c|}
\hline & $\mathrm{P}$ & $\mathrm{T}$ & Mass-flow & Mass & $\begin{array}{c}\text { Residence } \\
\text { time } \\
{[\mathrm{s}]}\end{array}$ & Volume \\
& {$[\mathrm{bar}]$} & {$[\mathrm{K}]$} & {$[\mathrm{kg} / \mathrm{s}]$} & {$[\mathrm{kg}]$} & 95 & 0.351 \\
\hline $\mathrm{VLP}^{(1)+} \mathrm{HX}^{(2)}$ & $0.016-0.4$ & $4-300$ & 0.126 & 12 & 95 & 0.02 \\
\hline $\mathrm{LP}$ & 1.3 & 20 & 0.126 & 0.06 & 0.5 & 0.02 \\
\hline $\mathrm{HP}$ & 20 & $300-30$ & 0.126 & 0.6 & 5 & 0.5 \\
\hline Sub-cooler & 0.016 & 1.8 & $=$ & 60 & $=$ & 0.4 \\
\hline Absorber & 20 & 80 & 0.126 & 2.3 & 20 & \\
\hline
\end{tabular}

(1) $\mathrm{VLP}=$ Very Low Pressure

(2) $\mathrm{HX}=$ heat exchanger

Table 11: Maximum He masses and volumes in the QUI

\begin{tabular}{|c|c|c|c|c|c|c|}
\hline & $\begin{array}{c}\mathrm{P} \\
{[\mathrm{bar}]}\end{array}$ & $\begin{array}{c}\mathrm{T} \\
{[\mathrm{K}]}\end{array}$ & $\begin{array}{c}\text { Mass-flow } \\
\qquad[\mathrm{kg} / \mathrm{s}]\end{array}$ & $\begin{array}{l}\text { Mass } \\
{[\mathrm{kg}]}\end{array}$ & $\begin{array}{c}\text { Residence } \\
\text { time } \\
{[\mathrm{s}]}\end{array}$ & $\begin{array}{c}\text { Volume } \\
\text { [m3] }\end{array}$ \\
\hline Header B & 0.016 & 4 & 0.126 & 0.1 & 1 & 0.6 \\
\hline Header C & 3.6 & 4.6 & 0.126 & 10 & 80 & 0.1 \\
\hline Header D & 1.3 & 20 & 0.126 & 0.6 & 4 & 0.2 \\
\hline Header E & 19.5 & 50 & 0.234 & 0.9 & 4 & 0.05 \\
\hline Header F & 18 & 75 & 0.234 & 0.6 & 2 & 0.05 \\
\hline Sub-cooler & 1 & 4.2 & $=$ & 63 & $=$ & 0.5 \\
\hline
\end{tabular}

Table 12: Maximum He masses and volumes in the QURA

\begin{tabular}{|l|c|c|c|c|c|c|}
\hline & $\mathrm{P}$ & $\mathrm{T}$ & Mass-flow & Mass & $\begin{array}{c}\text { Residence } \\
\text { time } \\
{[\mathrm{s}]}\end{array}$ & Volume \\
& {$[\mathrm{bar}]$} & {$[\mathrm{K}]$} & {$[\mathrm{kg} / \mathrm{s}]$} & {$[\mathrm{kg}]$} & 110 & 1 \\
\hline HP & 20 & $20-4.5$ & 0.8 & 88.17 & 220 & 2 \\
\hline LP & 1.3 & $4.5-20$ & 0.8 & 176.34 & $\infty$ & 1 \\
\hline Sub-cooler & 1.3 & 4.5 & $=$ & 120 & $\infty$ \\
\hline
\end{tabular}


Table 13: Maximum He masses and volumes in the interconnecting cryogenic lines and interconnecting warm pipes in the caverns and shafts

\begin{tabular}{|c|c|c|c|c|c|c|c|c|}
\hline \multicolumn{3}{|l|}{ Pipe } & \multirow{2}{*}{$\begin{array}{c}\mathrm{P} \\
\text { [bar] }\end{array}$} & \multirow{2}{*}{$\begin{array}{r}\mathrm{T} \\
{[\mathrm{K}]}\end{array}$} & \multirow{2}{*}{$\begin{array}{l}\text { Mass-flow } \\
{[\mathrm{kg} / \mathrm{s}]}\end{array}$} & \multirow{2}{*}{$\begin{array}{c}\text { Mass } \\
{[\mathrm{kg}]} \\
\end{array}$} & \multirow{2}{*}{$\begin{array}{l}\text { Residence } \\
\text { time }\end{array}$} & \multirow{2}{*}{$\begin{array}{l}\text { Volume } \\
\left.\mathrm{m}^{3}\right] \\
\end{array}$} \\
\hline from & To & code & & & & & & \\
\hline QUI & QURC & $\begin{array}{l}720 \text { (header B) } \\
720 \text { (header C) } \\
720 \text { (header D) }\end{array}$ & $\begin{array}{l}0.016 \\
3.6 \\
1.3\end{array}$ & $\begin{array}{l}4 \\
4.6 \\
20\end{array}$ & $\begin{array}{l}0.126 \\
0.126 \\
0.126\end{array}$ & $\begin{array}{l}0.04 \\
3.6 \\
0.2\end{array}$ & $\begin{array}{l}0.3 \\
28 \\
1.5\end{array}$ & $\begin{array}{l}0.2 \\
0.03 \\
0.06\end{array}$ \\
\hline QUI & QURC & $\begin{array}{l}760 \text { (header B) } \\
760 \text { (header C) } \\
760 \text { (header D) }\end{array}$ & $\begin{array}{l}0.016 \\
3.6 \\
1.3\end{array}$ & $\begin{array}{l}4 \\
4.6 \\
20\end{array}$ & $\begin{array}{l}0.126 \\
0.126 \\
0.126\end{array}$ & $\begin{array}{l}0.04 \\
3.6 \\
0.2\end{array}$ & $\begin{array}{l}0.3 \\
28 \\
1.5\end{array}$ & $\begin{array}{l}0.2 \\
0.03 \\
0.06\end{array}$ \\
\hline QUI & QURB & $\begin{array}{l}750 \text { (header C) } \\
750 \text { (header D) } \\
750 \text { (header E) } \\
750 \text { (header F) }\end{array}$ & $\begin{array}{l}3.6 \\
1.3 \\
19 \\
18\end{array}$ & $\begin{array}{l}4.6 \\
20 \\
50 \\
75\end{array}$ & $\begin{array}{l}0.235 \\
0.165 \\
0.24 \\
0.24\end{array}$ & $\begin{array}{l}174 \\
9.4 \\
15 \\
9.5\end{array}$ & $\begin{array}{l}740 \\
48 \\
60 \\
38\end{array}$ & $\begin{array}{l}1.3 \\
3 \\
0.8 \\
0.8\end{array}$ \\
\hline QUI & QURA & $\begin{array}{l}710 \text { (header C) } \\
710 \text { (header D) } \\
710 \text { (header E) } \\
710 \text { (header F) }\end{array}$ & $\begin{array}{l}3.6 \\
1.3 \\
19 \\
18\end{array}$ & $\begin{array}{l}4.6 \\
20 \\
50 \\
75\end{array}$ & $\begin{array}{l}0.126 \\
0.165 \\
0.24 \\
0.24\end{array}$ & $\begin{array}{l}10 \\
0.5 \\
0.9 \\
0.6\end{array}$ & $\begin{array}{l}81 \\
3.3 \\
3.7 \\
2.3\end{array}$ & $\begin{array}{l}0.1 \\
0.2 \\
0.05 \\
0.05\end{array}$ \\
\hline QUI & Surface $^{(1)}$ & 000 & 1 & 4 & $=$ & $=$ & $=$ & 5.3 \\
\hline QUI & $\mathrm{QSV}_{-}{ }^{(1)}$ & 210 & 20 & 4 & $=$ & $=$ & $=$ & 12.3 \\
\hline QURC & QSCC & $\begin{array}{l}121 \\
122\end{array}$ & $\begin{array}{l}15 \\
1\end{array}$ & $\begin{array}{l}300 \\
300\end{array}$ & $\begin{array}{l}0.126 \\
0.126\end{array}$ & $\begin{array}{l}5 \\
2\end{array}$ & $\begin{array}{l}36 \\
15\end{array}$ & $\begin{array}{l}2 \\
12.3\end{array}$ \\
\hline QURC & QSCC & $\begin{array}{l}161 \\
162\end{array}$ & $\begin{array}{l}15 \\
1\end{array}$ & $\begin{array}{l}300 \\
300\end{array}$ & $\begin{array}{l}0.126 \\
0.126\end{array}$ & $\begin{array}{l}5 \\
2\end{array}$ & $\begin{array}{l}36 \\
15\end{array}$ & $\begin{array}{l}2 \\
12.3\end{array}$ \\
\hline QURC & QSCA & $\begin{array}{l}141 \\
142\end{array}$ & $\begin{array}{l}15 \\
1\end{array}$ & $\begin{array}{l}300 \\
300\end{array}$ & $\begin{array}{l}0.031 \\
0.031\end{array}$ & $\begin{array}{l}3 \\
0.2\end{array}$ & $\begin{array}{l}97 \\
6.5\end{array}$ & $\begin{array}{l}1.3 \\
1.3\end{array}$ \\
\hline QURC & QSCB & $\begin{array}{l}181 \\
182\end{array}$ & $\begin{array}{l}15 \\
1\end{array}$ & $\begin{array}{l}300 \\
300\end{array}$ & $\begin{array}{l}0.041 \\
0.041\end{array}$ & $\begin{array}{l}3 \\
0.2\end{array}$ & $\begin{array}{l}73 \\
5\end{array}$ & $\begin{array}{l}1.3 \\
1.3\end{array}$ \\
\hline QURA & QSRA & $\begin{array}{l}210 \text { (HP) } \\
210 \text { (LP) } \\
210 \text { (header E) } \\
210 \text { (header F) }\end{array}$ & $\begin{array}{l}20 \\
1.3 \\
19.5 \\
18\end{array}$ & $\begin{array}{l}20 \\
20 \\
50 \\
75\end{array}$ & $\begin{array}{l}0.69 \\
0.66 \\
0.225 \\
0.225\end{array}$ & $\begin{array}{l}63 \\
9.4 \\
15.3 \\
9.5\end{array}$ & $\begin{array}{l}91 \\
14 \\
68 \\
42\end{array}$ & $\begin{array}{l}1.3 \\
3 \\
0.9 \\
0.9\end{array}$ \\
\hline QURA & QSCA & $\begin{array}{l}131 \\
132 \\
133 \\
134\end{array}$ & $\begin{array}{l}20 \\
20 \\
1 \\
1\end{array}$ & $\begin{array}{l}300 \\
300 \\
300 \\
300\end{array}$ & $\begin{array}{l}0.042 \\
0.050^{(2)} \\
0.042 \\
=\end{array}$ & $\begin{array}{l}6.6 \\
1.0 \\
0.3 \\
=\end{array}$ & $\begin{array}{l}158 \\
20 \\
8 \\
=\end{array}$ & $\begin{array}{l}2.1 \\
0.3 \\
2.1 \\
3.0\end{array}$ \\
\hline $\begin{array}{l}\text { QRP } \\
\text { (warm } \\
\text { recovery } \\
\text { line) }\end{array}$ & QSCA & 110 & 1.5 & 300 & 0.037 & 2.0 & 51 & 7.8 \\
\hline $\begin{array}{l}\text { QRP } \\
\text { (warm } \\
\text { recovery } \\
\text { line) }\end{array}$ & QSCB & 150 & 1.5 & 300 & 0.042 & 2.0 & 54 & 7.8 \\
\hline $\begin{array}{l}\text { QRP } \\
\left(\begin{array}{l}\mathrm{He} \quad \text { ring } \\
\text { line })\end{array}\right.\end{array}$ & $\mathrm{QSV}_{-}$ & 030 & 20 & 300 & $=$ & 25 & $=$ & 7.8 \\
\hline
\end{tabular}

(1): pipe used in case of emergency

(2): pipe used only during warm-up 


\subsection{Potential and credible failure modes at the nodes located in the caverns and shafts}

All potential failures at the cryogenic nodes located in the caverns and shafts, which may happen at any phase of the collider operation, are listed in Table 14 and Table 15. In a similar ways as for the nodes located in the tunnel, we identify a failure at a node by the following code: Ux.y or Px.y, where U and P stand for location [4], x stands for element number (see Table 10) and y stands for failure mode number (see Table 1). 
II

DXX

क

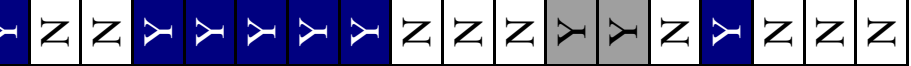

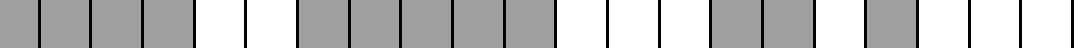

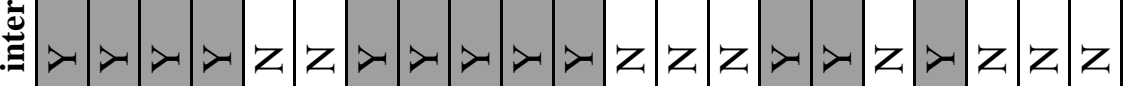

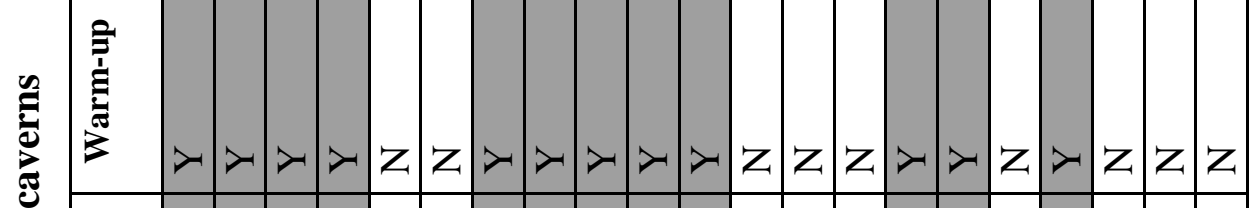

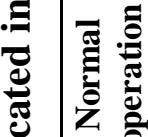

。

$\stackrel{0}{0}$

के

I

寻资

항

串

窇

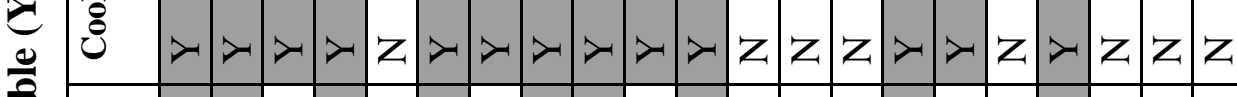

己ृ

$\frac{2}{\frac{1}{2}}$

든

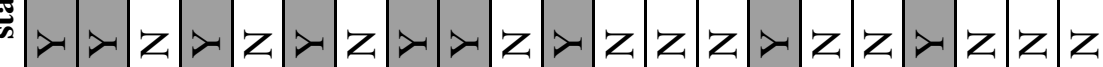

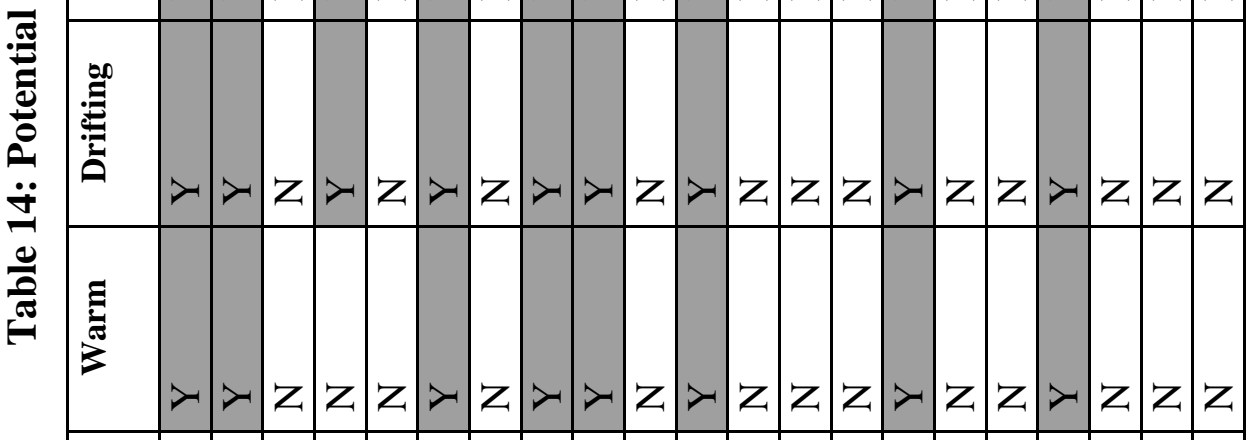

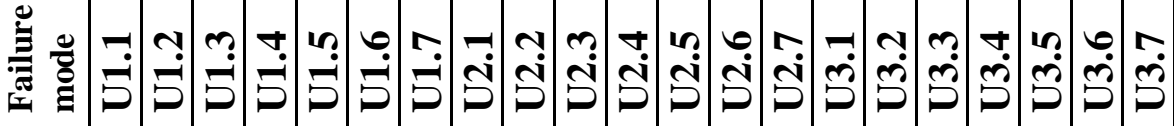

\begin{tabular}{|l|l|l|l|}
\hline \multirow{2}{*}{} & & & \\
$z$ & & & \\
& $S$ & $S$ & $\rho$ \\
\hline
\end{tabular}




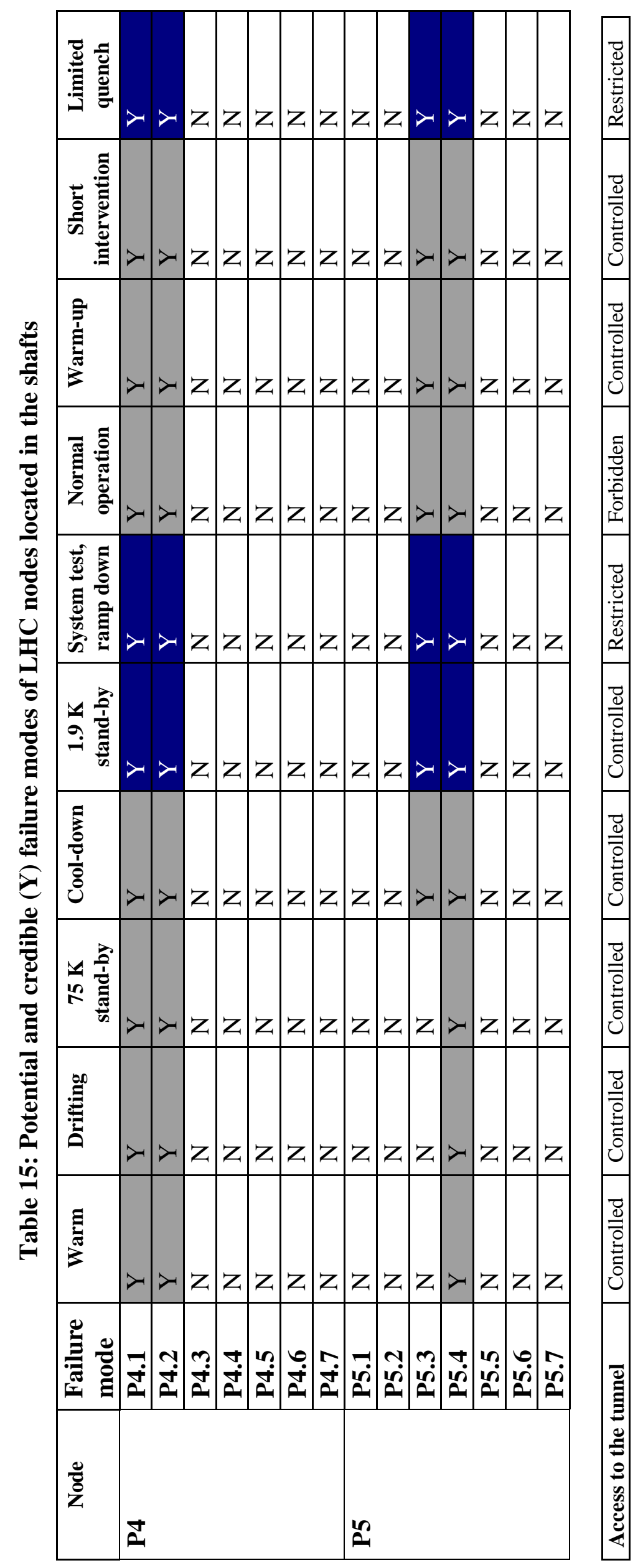




\subsection{Analysis of and recommendations for credible failure modes at the nodes located in the caverns and shafts}

The credible failures of the LHC cryogenic elements located in caverns and shafts and their probable causes are listed in Table 16.

Table 17 gives physical consequences of the failures, proposes the means of failure detection, as well as preventive and corrective defence measures.

\section{Table 16: Credible failures of the machine elements located in caverns and shafts, and their causes}

\begin{tabular}{|c|c|c|}
\hline Element & Failure & Probable causes \\
\hline \multirow[t]{5}{*}{$\begin{array}{l}\text { U1. Cold compressor boxes } \\
\text { (QURC) }\end{array}$} & $\begin{array}{l}\text { U1.1 Air flow to QURC insulation } \\
\text { vacuum }\end{array}$ & $\begin{array}{l}\text { Mechanical impact on a vacuum } \\
\text { tapping } \\
\text { Bellows failure } \\
\text { Weld non-tight } \\
\end{array}$ \\
\hline & $\begin{array}{l}\text { U1.2 Helium flow to QURC } \\
\text { insulation vacuum }\end{array}$ & $\begin{array}{l}\text { Bellows failure } \\
\text { Weld non-tight }\end{array}$ \\
\hline & $\begin{array}{l}\text { U1.3 Air flow to sub-atmospheric } \\
\text { helium }\end{array}$ & Break of helium guard \\
\hline & U1.4 Helium flow to environment & Break of instrumentation capillary \\
\hline & $\begin{array}{l}\text { U1.7 Pressurised helium flow to } \\
\text { subatmospheric helium }\end{array}$ & $\begin{array}{l}\text { Break of heat exchanger, leaking } \\
\text { valve }\end{array}$ \\
\hline \multirow[t]{4}{*}{$\begin{array}{l}\text { U2. Cryogenic interconnection } \\
\text { boxes (QUI) }\end{array}$} & $\begin{array}{l}\text { U2.1 Air flow to QUI insulation } \\
\text { vacuum }\end{array}$ & $\begin{array}{l}\text { Mechanical impact on a vacuum } \\
\text { tapping } \\
\text { Bellows failure } \\
\text { Weld non-tight } \\
\end{array}$ \\
\hline & $\begin{array}{l}\text { U2.2 Helium flow to QUI } \\
\text { insulation vacuum }\end{array}$ & $\begin{array}{l}\text { Break of a bellow } \\
\text { Weld non-tight }\end{array}$ \\
\hline & $\begin{array}{l}\text { U2.3 Air flow to sub-atmospheric } \\
\text { helium }\end{array}$ & Break of helium guard \\
\hline & U2.4 Helium flow to environment & Break of instrumentation capillary \\
\hline \multirow[t]{3}{*}{$\begin{array}{l}\text { U3. Underground Lower Cold } \\
\text { Boxes (QURA) }\end{array}$} & $\begin{array}{l}\text { U3.1. Air flow to QURA insulation } \\
\text { vacuum }\end{array}$ & $\begin{array}{l}\text { Mechanical impact on a vacuum } \\
\text { tapping } \\
\text { Bellows failure } \\
\text { Weld non-tight }\end{array}$ \\
\hline & $\begin{array}{l}\text { U3.2 Helium flow to QURA } \\
\text { insulation vacuum }\end{array}$ & $\begin{array}{l}\text { Bellows failure } \\
\text { Weld non-tight }\end{array}$ \\
\hline & U3.4. Helium flow to environment & Break of instrumentation capillary \\
\hline \multirow[t]{2}{*}{ P4. Interconnecting cryogenic lines } & $\begin{array}{l}\text { P4.1 Air flow to the transfer line } \\
\text { insulation vacuum }\end{array}$ & $\begin{array}{l}\text { Mechanical impact on a vacuum } \\
\text { tapping } \\
\text { Bellows failure } \\
\text { Weld non-tight } \\
\end{array}$ \\
\hline & $\begin{array}{l}\text { P4.2 Helium flow to the transfer } \\
\text { line insulation vacuum }\end{array}$ & $\begin{array}{l}\text { Bellows failure } \\
\text { Weld non-tight }\end{array}$ \\
\hline \multirow[t]{2}{*}{ P5. Interconnecting warm pipes } & $\begin{array}{l}\text { U5.3 Air flow to subatmospheric } \\
\text { helium }\end{array}$ & Break of pipe \\
\hline & U5.4 Helium flow to environment & $\begin{array}{l}\text { Break of pipe due to e. g. ice } \\
\text { condensation }\end{array}$ \\
\hline
\end{tabular}




\begin{tabular}{|c|c|c|c|}
\hline لِّ & 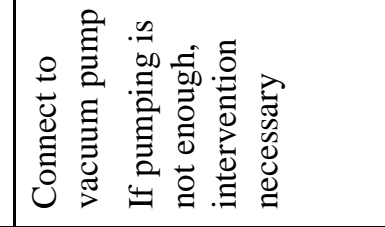 & 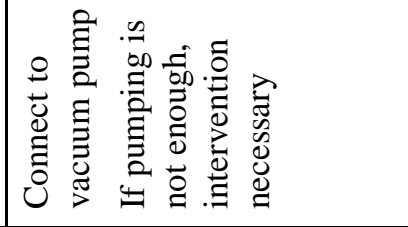 & 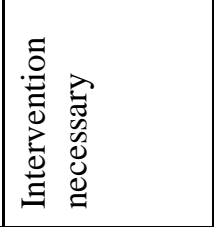 \\
\hline 萢 & 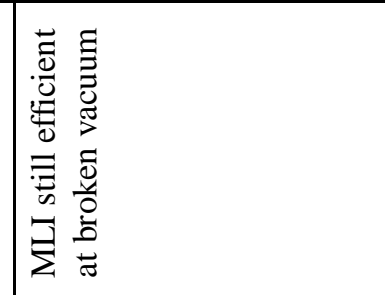 & 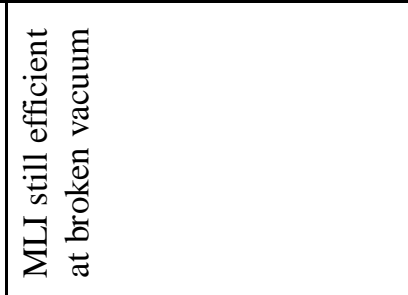 & 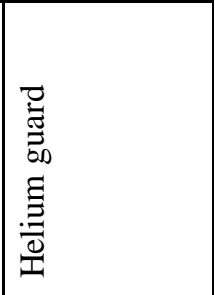 \\
\hline "ू & 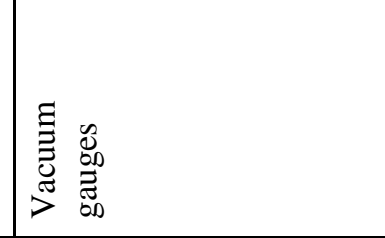 & 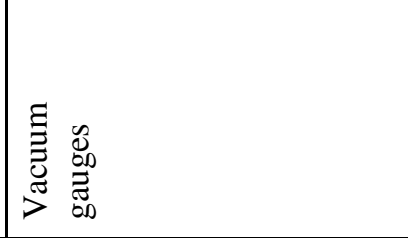 & 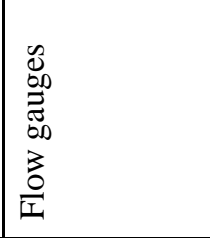 \\
\hline 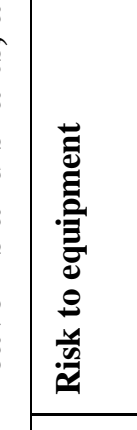 & 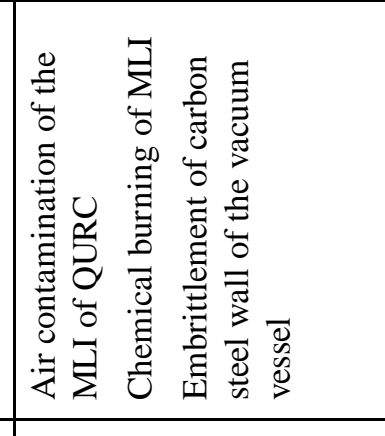 & 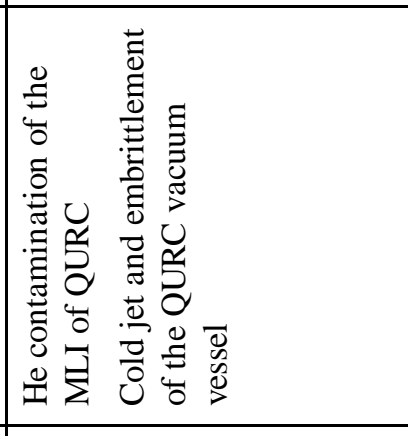 & 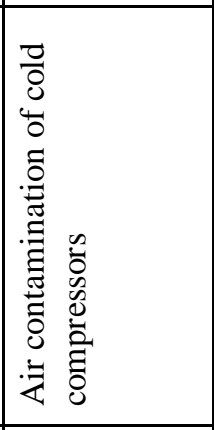 \\
\hline 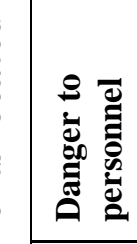 & 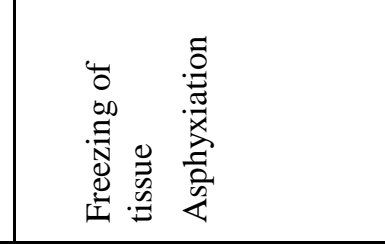 & 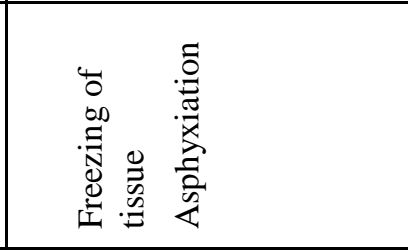 & 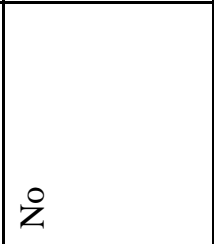 \\
\hline 总 & 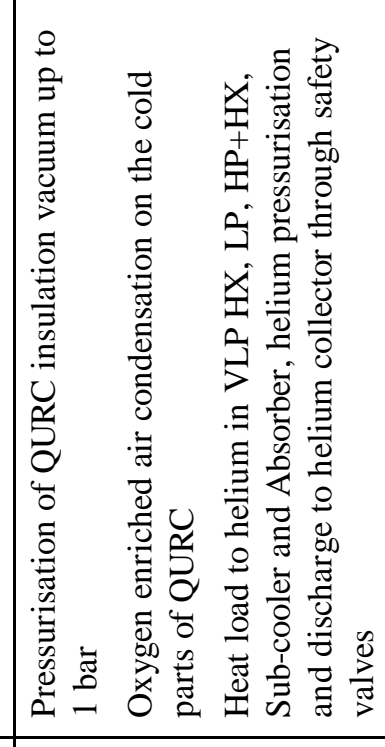 & 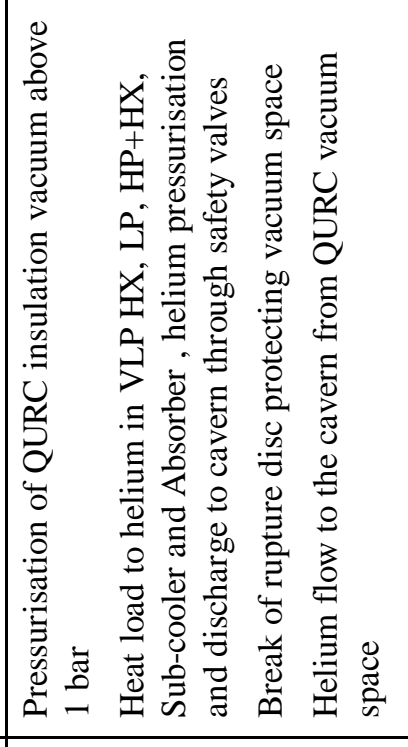 & 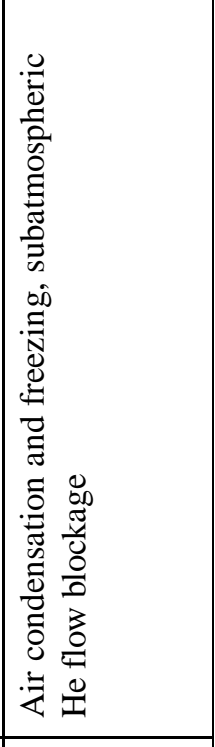 \\
\hline 童 & 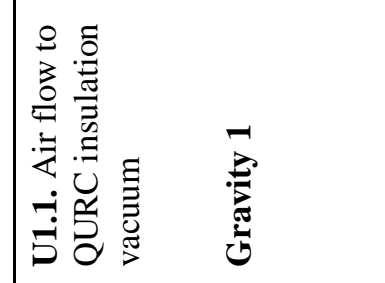 & 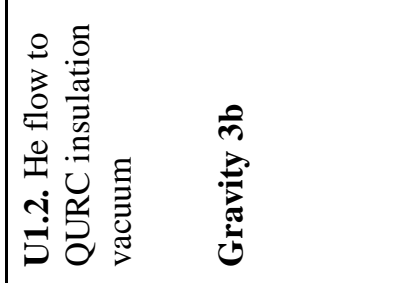 & 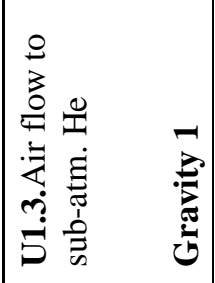 \\
\hline
\end{tabular}




\begin{tabular}{|c|c|c|c|c|c|}
\hline 菢 & 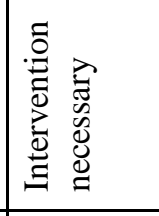 & 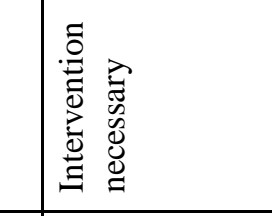 & 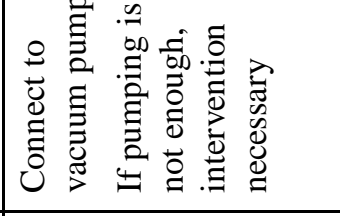 & 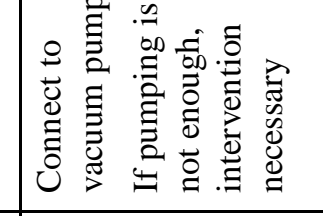 & 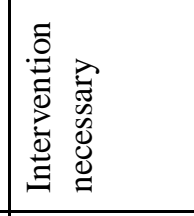 \\
\hline 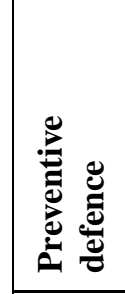 & 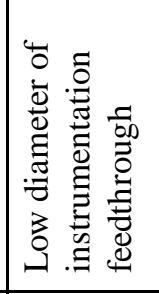 & 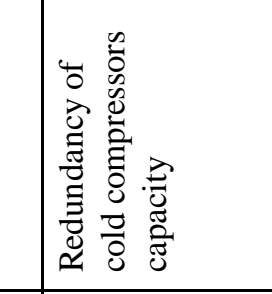 & 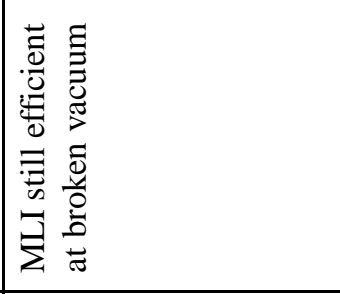 & 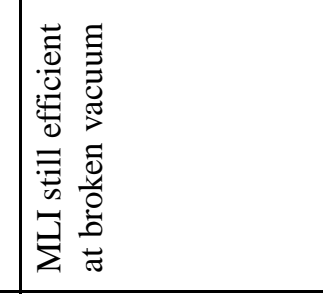 & 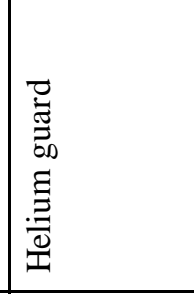 \\
\hline 列 & 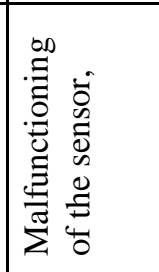 & 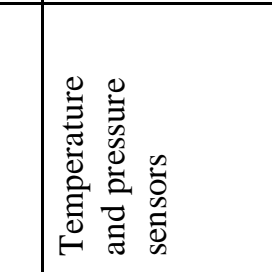 & 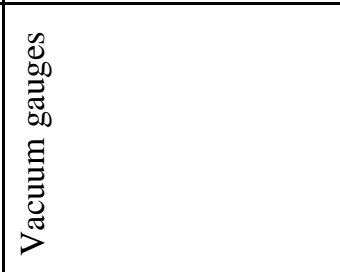 & 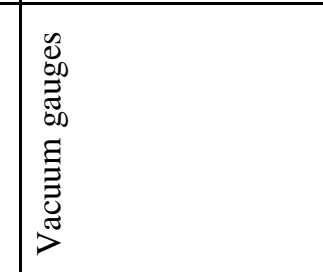 & 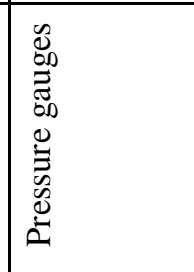 \\
\hline 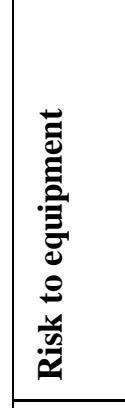 & そ & $\stackrel{8}{z}$ & 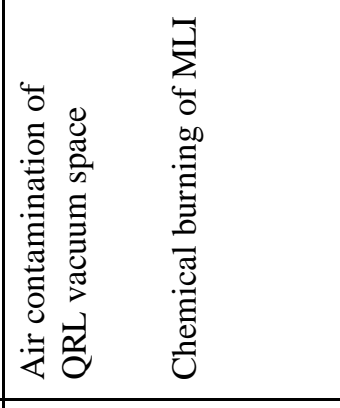 & 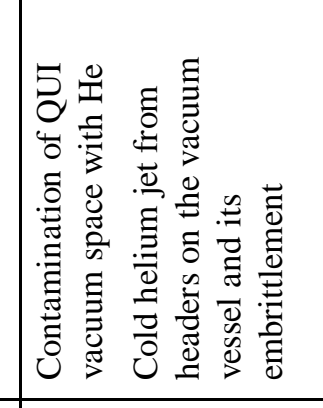 & 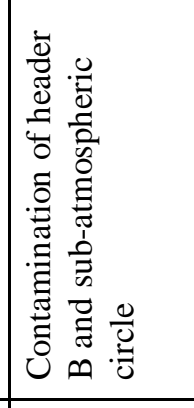 \\
\hline 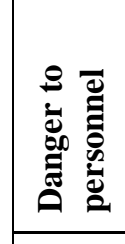 & 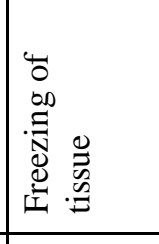 & z & 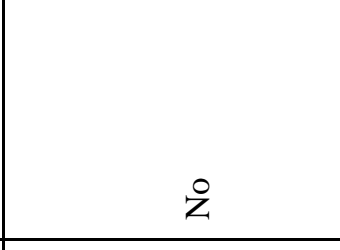 & 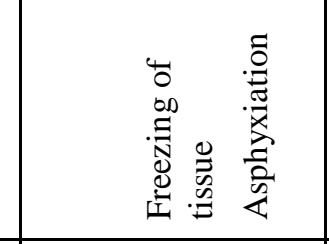 & z \\
\hline 总 & 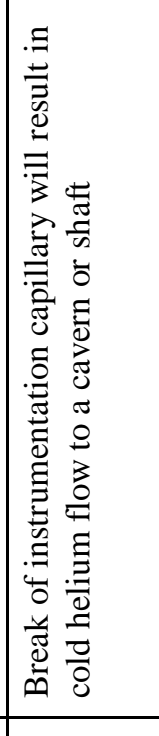 & 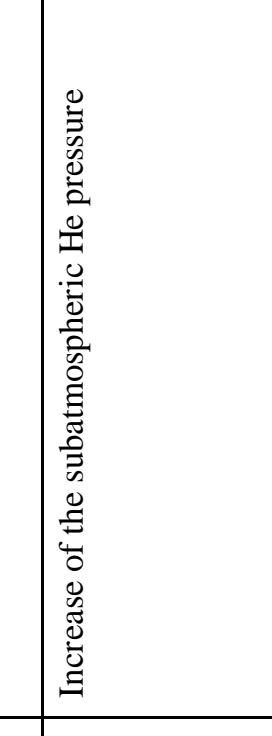 & 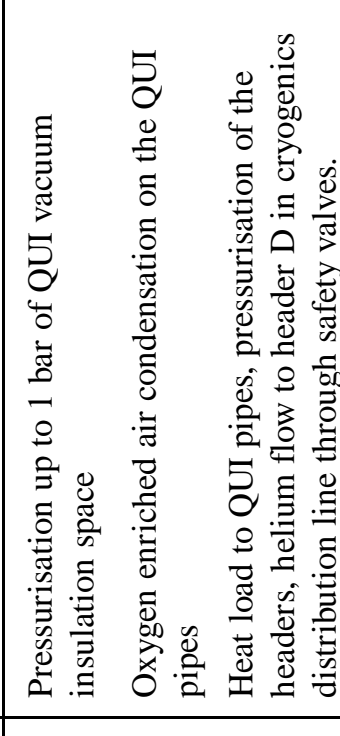 & 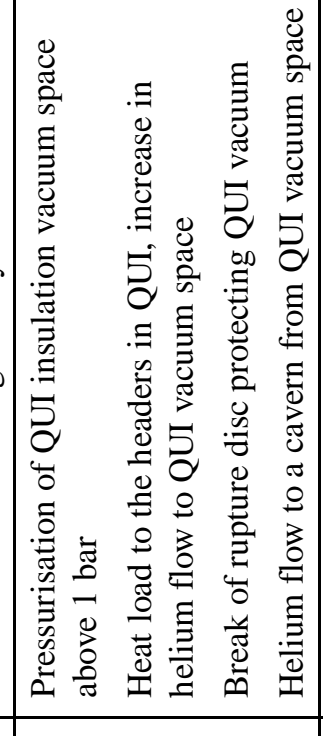 & 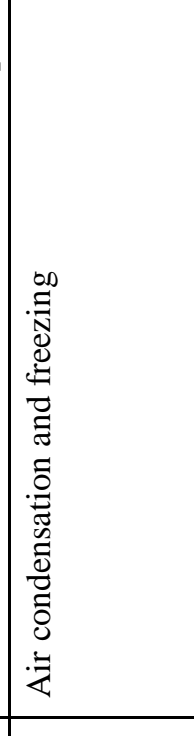 \\
\hline 童 & 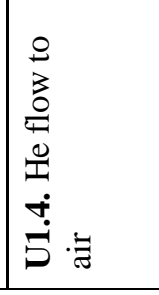 & 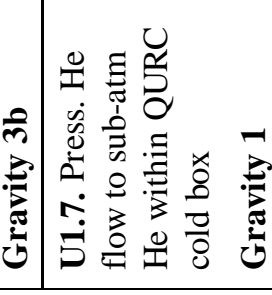 & 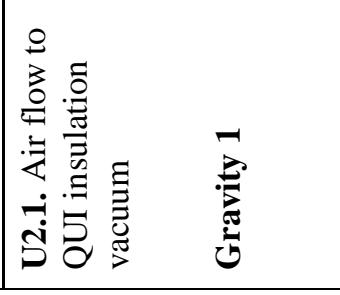 & 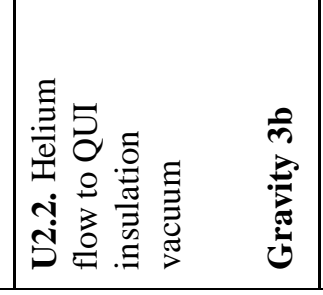 & 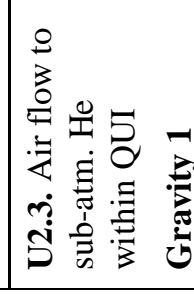 \\
\hline
\end{tabular}




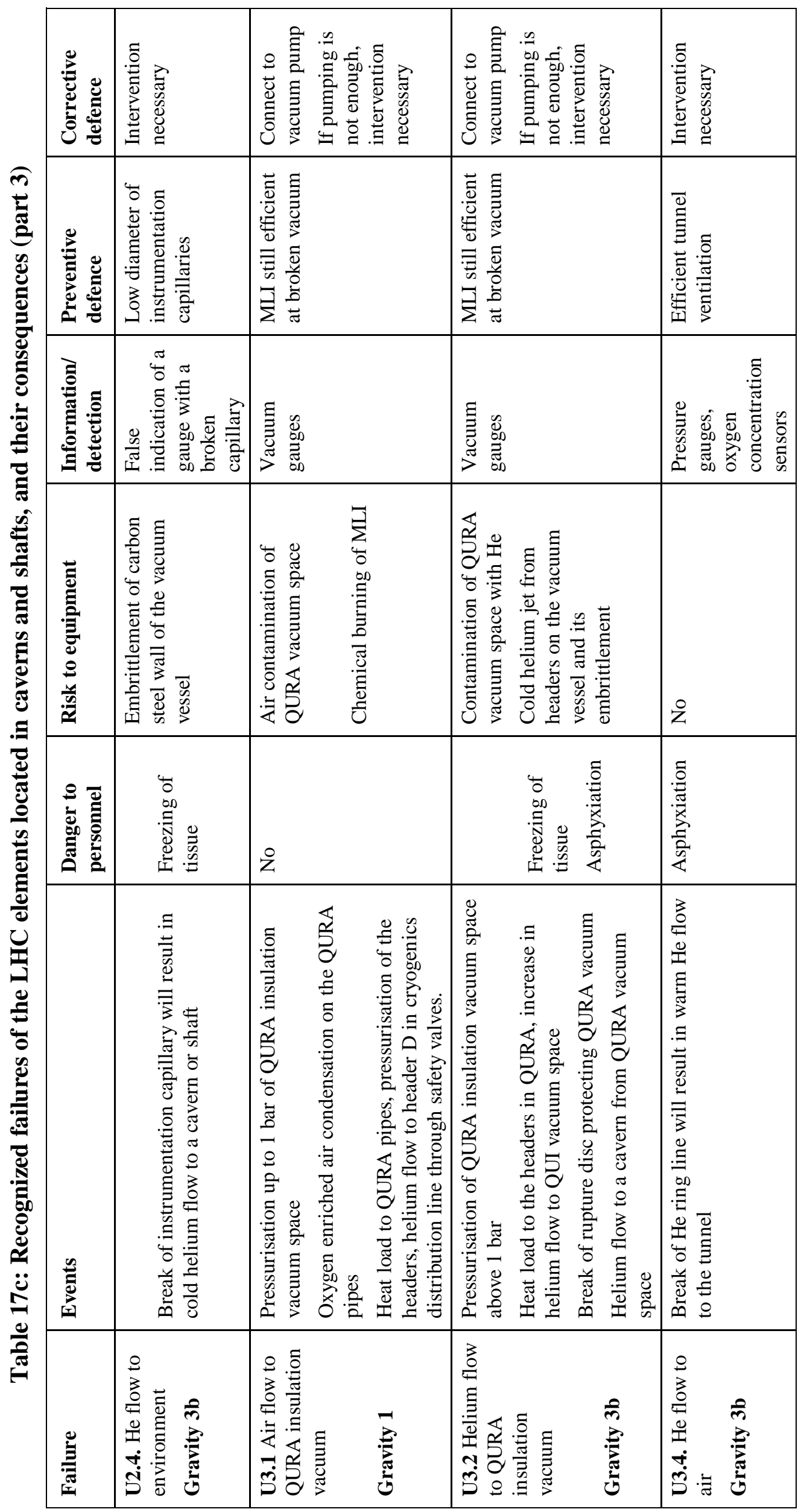




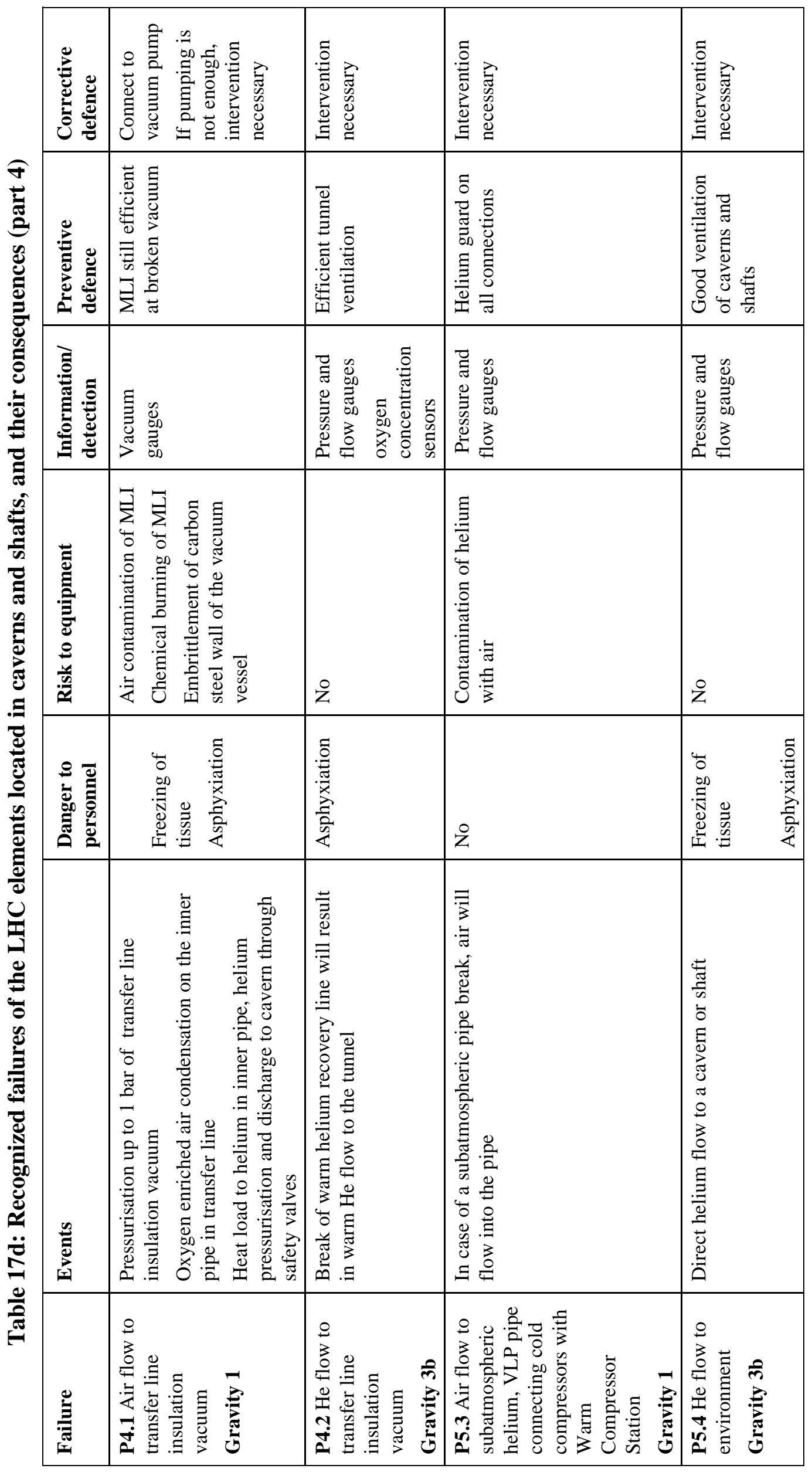


There are 8 events of gravity $3 \mathrm{~b}$ identified in Table 17. Table 18 lists the $3 \mathrm{~b}$ gravity events, gives the highest amounts of helium that might be relieved to the caverns and shaft together with an approximate mass flow rates and formulates the recommendations.

Table 18: Gravity $3 b$ failures at the nodes located in caverns and shafts

\begin{tabular}{|c|c|c|}
\hline Failure & $\begin{array}{l}\text { Maximum amount of helium } \\
\text { relieved }[\mathrm{kg}] \text { / approximate flow } \\
\text { rate }[\mathrm{kg} / \mathrm{s}]\end{array}$ & Recommendations \\
\hline $\begin{array}{l}\text { U1.2. He flow to QURC insulation } \\
\text { vacuum } \\
\text { Gravity } 3 \mathbf{b}\end{array}$ & 60 / below 2 & $\begin{array}{l}\text { Consequences of the failure mode } \\
\text { fully covered by U3.2 }\end{array}$ \\
\hline $\begin{array}{l}\text { U1.4. He flow from QURC to } \\
\text { environment } \\
\text { Gravity } \mathbf{3 b}\end{array}$ & $60 /$ of the leak order & $\begin{array}{l}\text { Consequences of the failure mode } \\
\text { fully covered by U3.2 }\end{array}$ \\
\hline $\begin{array}{l}\text { U2.2. Helium flow to QUI } \\
\text { insulation vacuum } \\
\text { Gravity } \mathbf{3 b}\end{array}$ & 63 / below 2 & $\begin{array}{l}\text { Consequences of the failure mode } \\
\text { fully covered by U3.2 }\end{array}$ \\
\hline $\begin{array}{l}\text { U2.4. He flow from QUI } \\
\text { enclosures to environment } \\
\text { Gravity } \mathbf{3 b}\end{array}$ & 63 / of the leak order & $\begin{array}{l}\text { Consequences of the failure mode } \\
\text { fully covered by U } 3.2\end{array}$ \\
\hline $\begin{array}{l}\text { U3.2 Helium flow to QURA } \\
\text { insulation vacuum, LP circuit } \\
\text { break }\end{array}$ & 176 kg / below 2 & Worst case scenario \\
\hline $\begin{array}{l}\text { U3.4. He flow from QURA to } \\
\text { environment }\end{array}$ & $176 \mathrm{~kg} /$ of the leak order & $\begin{array}{l}\text { Consequences of the failure mode } \\
\text { fully covered by U } 3.2\end{array}$ \\
\hline $\begin{array}{l}\text { P4.2 He flow from header C of the } \\
\text { interconnecting cryogenic line } \\
\text { between QUI and QURB to its } \\
\text { insulation vacuum }\end{array}$ & $174 \mathrm{~kg} /$ below 2 & $\begin{array}{l}\text { From the consequences failure } \\
\text { similar to U3.2 }\end{array}$ \\
\hline $\begin{array}{l}\text { P5.4 He flow from QRP (warm } \\
\text { recovery line) to environment }\end{array}$ & $2 \mathrm{~kg} /$ of the leak order & $\begin{array}{l}\text { Consequences of the failure mode } \\
\text { fully covered by U } 3.2\end{array}$ \\
\hline
\end{tabular}

As it follows from Table 18, the worst case scenario that may happen in a cavern or a shaft, is helium flow to QURA insulation vacuum due to the break of low pressure circuit (1.3 bar). The event will be followed by relief of about $176 \mathrm{~kg}$ of helium directly to the atmosphere of a cavern or shaft. 


\section{Risks associated at failures of LHC cryogenic system nodes at the surface}

\subsection{LHC cryogenic system nodes located at the surface (buildings and open space)}

The elements of cryogenic systems, which are located at the surface are listed in Table 19.

Table 19: LHC cryogenic system nodes located at the surface

\begin{tabular}{|l|l|l|}
\hline Node & Description & Remarks \\
\hline S1 & Surface upper cold boxes (QSRA) & Buildings \\
\hline S2 & Surface integrated cold boxes (QSRB) & Buildings \\
\hline S3 & $\begin{array}{l}\text { Helium compressor stations (QSCA, QSCB, } \\
\text { QSCC) }\end{array}$ & Buildings \\
\hline S4 & Interconnecting cryogenic lines (QSL_N, QSL_H) & Buildings \\
\hline S5 & $\begin{array}{l}\text { Interconnecting warm pipes (QSPA, QSPB, QPPA, } \\
\text { QPPB, QSPD) }\end{array}$ & Buildings \\
\hline S6 & $\begin{array}{l}\text { Helium storage medium-pressure tanks (QSVA, } \\
\text { QSVB) }\end{array}$ & Open space \\
\hline S7 & Liquid nitrogen storage (QSD_N) & Open space \\
\hline S8 & Liquid helium storage (QSD_H) & Open space \\
\hline S9 & Interconnecting cryogenic lines (QSL_N, QSL_H) & Open space \\
\hline S10 & Interconnecting warm pipes (QSPD) & Open space \\
\hline
\end{tabular}

A typical surface layout showing the buildings housing the cold boxes and the helium compressor stations, as well as the helium and nitrogen storages is presented in Figure 12. The amount of helium and volume of vacuum space involved in case of failure of the elements at the surface are given in Table 20 to Table 25. 


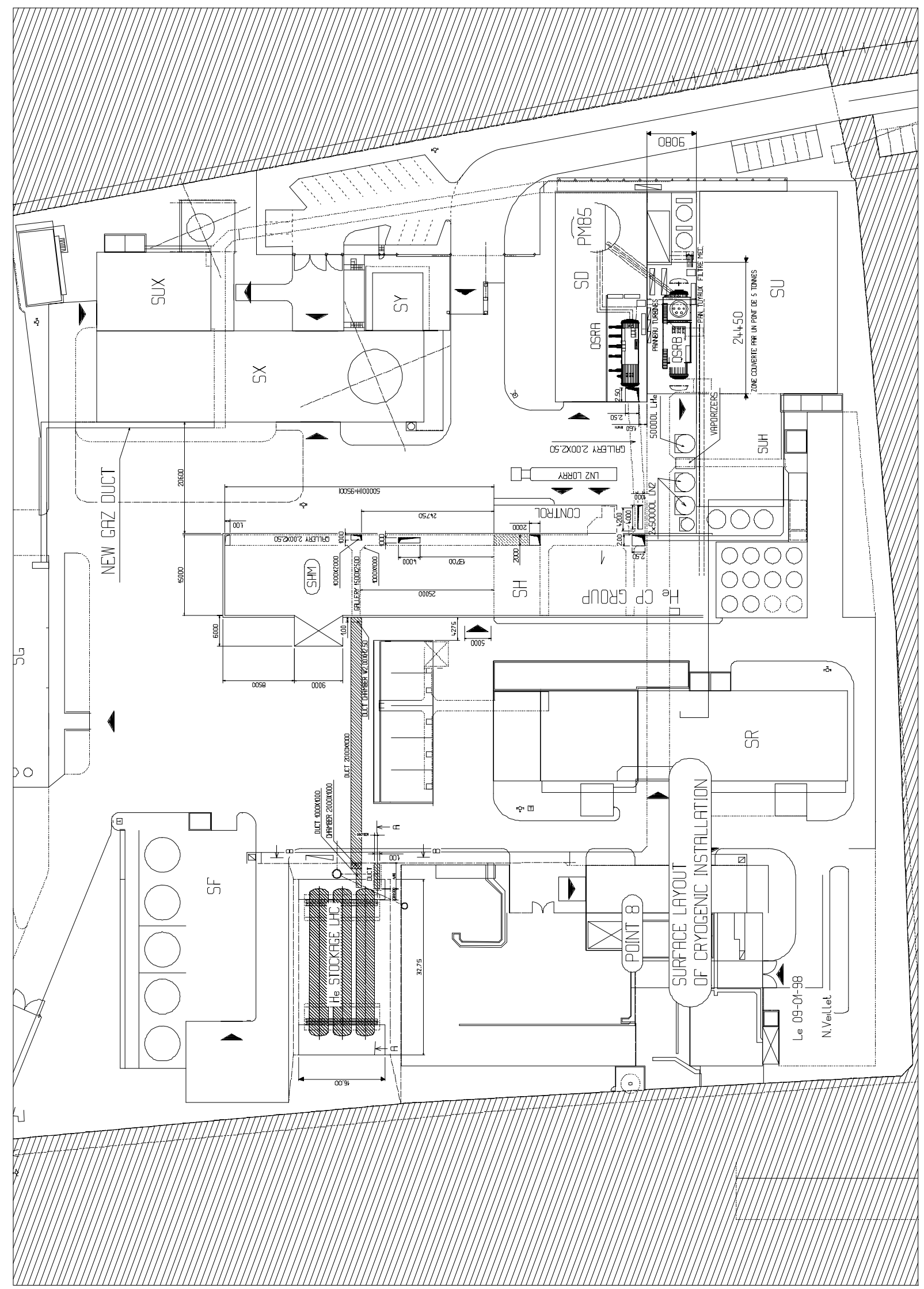

Figure 12: Typical layout of cryogenic equipment at the surface (P8) 
Table 20: Maximum He masses and volumes in the QSRA

\begin{tabular}{|l|c|c|c|c|c|c|}
\hline & $\mathrm{P}$ & $\mathrm{T}$ & Mass-flow & Mass & $\begin{array}{c}\text { Residence } \\
\text { time } \\
{[\mathrm{s}]}\end{array}$ & Volume \\
& {$[\mathrm{bar}]$} & {$[\mathrm{K}]$} & {$[\mathrm{kg} / \mathrm{s}]$} & {$[\mathrm{kg}]$} & 4 & 8 \\
\hline LP & 1.3 & $20-300$ & 0.800 & 3.33 & 4 & 4 \\
\hline MP & 4.5 & $20-300$ & 0.800 & 5.75 & 7 & 3 \\
\hline HP & 20 & $300-20$ & 1.600 & 18.89 & 1 & 1 \\
\hline Adsorber 1 & 20 & 80 & 1.200 & 11.62 & 10 & 0.2 \\
\hline Adsorber 2 & 20 & 20 & 0.800 & 9.41 & 1 & 4 \\
\hline
\end{tabular}

Table 21: Maximum He masses and volumes in the QSRB

\begin{tabular}{|l|c|c|c|c|c|c|}
\hline & $\mathrm{P}$ & $\mathrm{T}$ & Mass-flow & Mass & $\begin{array}{c}\text { Residence } \\
\text { time } \\
{[\mathrm{s}]}\end{array}$ & Volume \\
& {$[\mathrm{bar}]$} & {$[\mathrm{K}]$} & {$[\mathrm{kg} / \mathrm{s}]$} & {$[\mathrm{kg}]$} & 6 & 10 \\
\hline LP & 1.3 & $4.5-300$ & 0.740 & 4.17 & 7 & 5 \\
\hline MP & 4.5 & $20-300$ & 0.960 & 6.74 & 2 & 4 \\
\hline HP & 22 & $300-4.5$ & 1.700 & 27.65 & 10 & 1.2 \\
\hline Adsorber 1 & 22 & 80 & 1.460 & 15.29 & 10 & 0.1 \\
\hline Adsorber 2 & 22 & 20 & 0.740 & 5.14 & 7 & 2 \\
\hline Sub-cooler & 1.3 & 4.5 & 0.490 & 190.35 & 388 & \\
\hline
\end{tabular}

Table 22: Maximum He masses and volumes in the QSCA or QSCB

\begin{tabular}{|c|c|c|c|c|c|c|}
\hline & $\begin{array}{c}\mathrm{P} \\
\text { [bar] }\end{array}$ & $\begin{array}{c}\mathrm{T} \\
{[\mathrm{K}]}\end{array}$ & $\begin{array}{c}\text { Mass-flow } \\
\qquad[\mathrm{kg} / \mathrm{s}]\end{array}$ & $\begin{array}{l}\text { Mass } \\
{[\mathrm{kg}]}\end{array}$ & $\begin{array}{c}\text { Residence } \\
\text { time } \\
{[\mathrm{s}]}\end{array}$ & $\begin{array}{c}\text { Volume } \\
\text { [m3] }\end{array}$ \\
\hline LP & 1 & 300 & 0.800 & 3.05 & 3.809 & 19 \\
\hline MP & 4 & 300 & 0.800 & 2.88 & 3.604 & 4.5 \\
\hline $\mathrm{HP}$ & 20 & 300 & 1.600 & 14.31 & 8.945 & 4.5 \\
\hline Oil separator 1 & 20 & 360 & 1.600 & 31.86 & 19.911 & 12.0 \\
\hline Oil separator 2 & 4 & 360 & 0.800 & 3.74 & 4.673 & 7.0 \\
\hline Coalesher & 20 & 300 & 1.600 & 22.26 & 13.914 & 7.0 \\
\hline Adsorber & 20 & 300 & 1.600 & 3.18 & 1.988 & 1.0 \\
\hline
\end{tabular}


Table 23: Maximum He masses and volumes in the QSCC

\begin{tabular}{|l|c|c|c|c|c|c|}
\hline & $\mathrm{P}$ & $\mathrm{T}$ & Mass-flow & Mass & $\begin{array}{c}\text { Residence } \\
\text { time } \\
{[\mathrm{s}]}\end{array}$ & Volume \\
& {$[\mathrm{bar}]$} & {$[\mathrm{K}]$} & {$[\mathrm{kg} / \mathrm{s}]$} & {$[\mathrm{kg}]$} & 0.13 \\
\hline LP & 4 & 300 & 0.126 & 0.06 & 0.493 & 0.1 \\
\hline HP & 0.34 & 300 & 0.126 & 0.03 & 0.210 & 0.5 \\
\hline Oil separator & 4 & 330 & 0.126 & 1.92 & 14.786 & 3 \\
\hline Coaleser & 4 & 300 & 0.126 & 0.32 & 2.464 & 0.5 \\
\hline Adsorber & 4 & 300 & 0.126 & 0.32 & 2.464 & 0.5 \\
\hline
\end{tabular}

Table 24: Maximum helium and nitrogen masses and volumes in the storage vessels

\begin{tabular}{|l|c|c|c|c|}
\hline & $\begin{array}{c}\mathrm{P} \\
{[\mathrm{bar}]}\end{array}$ & $\begin{array}{c}\mathrm{T} \\
{[\mathrm{K}]}\end{array}$ & $\begin{array}{c}\text { Mass } \\
{[\mathrm{kg}]}\end{array}$ & $\begin{array}{c}\text { Volume } \\
{[\mathrm{m} 3]}\end{array}$ \\
\hline HP & 20 & 300 & 795.08 & 250 \\
\hline LN2 & 3 & 80 & 40000 & 50 \\
\hline LHe & 3.5 & 4.5 & 6582.74 & 50 \\
\hline
\end{tabular}


Table 25: Maximum helium and nitrogen masses and volumes in interconnecting cryogenic lines and warm piping at the surface

\begin{tabular}{|c|c|c|c|c|c|c|c|c|}
\hline \multicolumn{3}{|l|}{ Pipe } & \multirow{2}{*}{$\begin{array}{r}\mathrm{P} \\
\text { [bar] }\end{array}$} & \multirow{2}{*}{$\begin{array}{r}\mathrm{T} \\
{[\mathrm{K}]}\end{array}$} & \multirow{2}{*}{$\begin{array}{c}\begin{array}{c}\text { Mass- } \\
\text { flow }\end{array} \\
{[\mathrm{kg} / \mathrm{s}]}\end{array}$} & \multirow{2}{*}{$\begin{array}{l}\text { Mass } \\
{[\mathrm{kg}]}\end{array}$} & \multirow{2}{*}{$\begin{array}{l}\begin{array}{c}\text { Residence } \\
\text { time }\end{array} \\
{[\mathrm{s}]}\end{array}$} & \multirow{2}{*}{$\begin{array}{l}\text { Volume } \\
{\left[\mathrm{m}^{3}\right]}\end{array}$} \\
\hline from & To & code & & & & & & \\
\hline \multirow[t]{3}{*}{ QSRA } & QSCA & $111(\mathrm{HP})$ & 20 & 300 & 1.4 & 10 & 7 & 3.1 \\
\hline & & $112(\mathrm{MP})$ & 4.5 & 300 & 0.75 & 2.3 & 3 & 3.1 \\
\hline & & 113 (LP) & 1.3 & 300 & 0.77 & 2.6 & 3 & 12.6 \\
\hline \multirow[t]{3}{*}{ QSRB } & QSCB & $151(\mathrm{HP})$ & 22 & 300 & 1.65 & 16.5 & 10 & 4.7 \\
\hline & & 152 (MP) & 4.5 & 300 & 0.85 & 3.4 & 4 & 4.7 \\
\hline & & 152 (LP) & 1.3 & 300 & 0.8 & 3.9 & 5 & 18.8 \\
\hline \multirow[t]{4}{*}{ QSRA } & QSCA & 131 & 20 & 300 & 0.042 & 3.9 & 93 & 1.2 \\
\hline & & 132 & 20 & 300 & $0.050^{(2)}$ & 0.62 & 12.4 & 0.2 \\
\hline & & 133 & 1 & 300 & 0.042 & 0.20 & 5 & 1.2 \\
\hline & & $134^{(1)}$ & 1 & 300 & $=$ & $=$ & $=$ & 3.0 \\
\hline \multirow[t]{3}{*}{ QSCA } & QSCB & 101 & 20 & 300 & 1.65 & 0.39 & 0.24 & 0.1 \\
\hline & & 102 & 4.5 & 300 & 0.85 & 0.23 & 0.27 & 0.3 \\
\hline & & 103 & 1.3 & 300 & 0.8 & 0.07 & 0.08 & 0.3 \\
\hline QSCA & QSV & 011 & 20 & 300 & 0.2 & 0.16 & 0.80 & 0.05 \\
\hline QSD_N & QSRA & 421 & 3 & 77 & 1.1 & 122 & 111 & 0.15 \\
\hline QSD_N & QSRB & 461 & 3 & 77 & 1.1 & 122 & 111 & 0.15 \\
\hline QSD_N & QSCA & 411 & 3 & 77 & 0.15 & 406 & 2708 & 0.50 \\
\hline QSD_N & QSCB & 451 & 3 & 77 & 0.15 & 406 & 2708 & 0.50 \\
\hline QSD_H & QSRB & 051 & 3.5 & 4.2 & 0.5 & 32 & 64 & 0.24 \\
\hline
\end{tabular}

(1): pipe used in case of emergency

(2): pipe used only during warm-up 


\subsection{Potential and credible failures at the nodes located at the surface}

All potential failures at the cryogenic nodes located at the surface, which may happen at any phase of the collider operation, are listed in Table 26. In a similar way as for the nodes located in the tunnel, caverns and shafts, we identify a failure at a node by the following code: Sx.y, where $S$ stands for location, $x$ stands for node number (see Table 19) and y stands for failure mode number (see Table 1) 


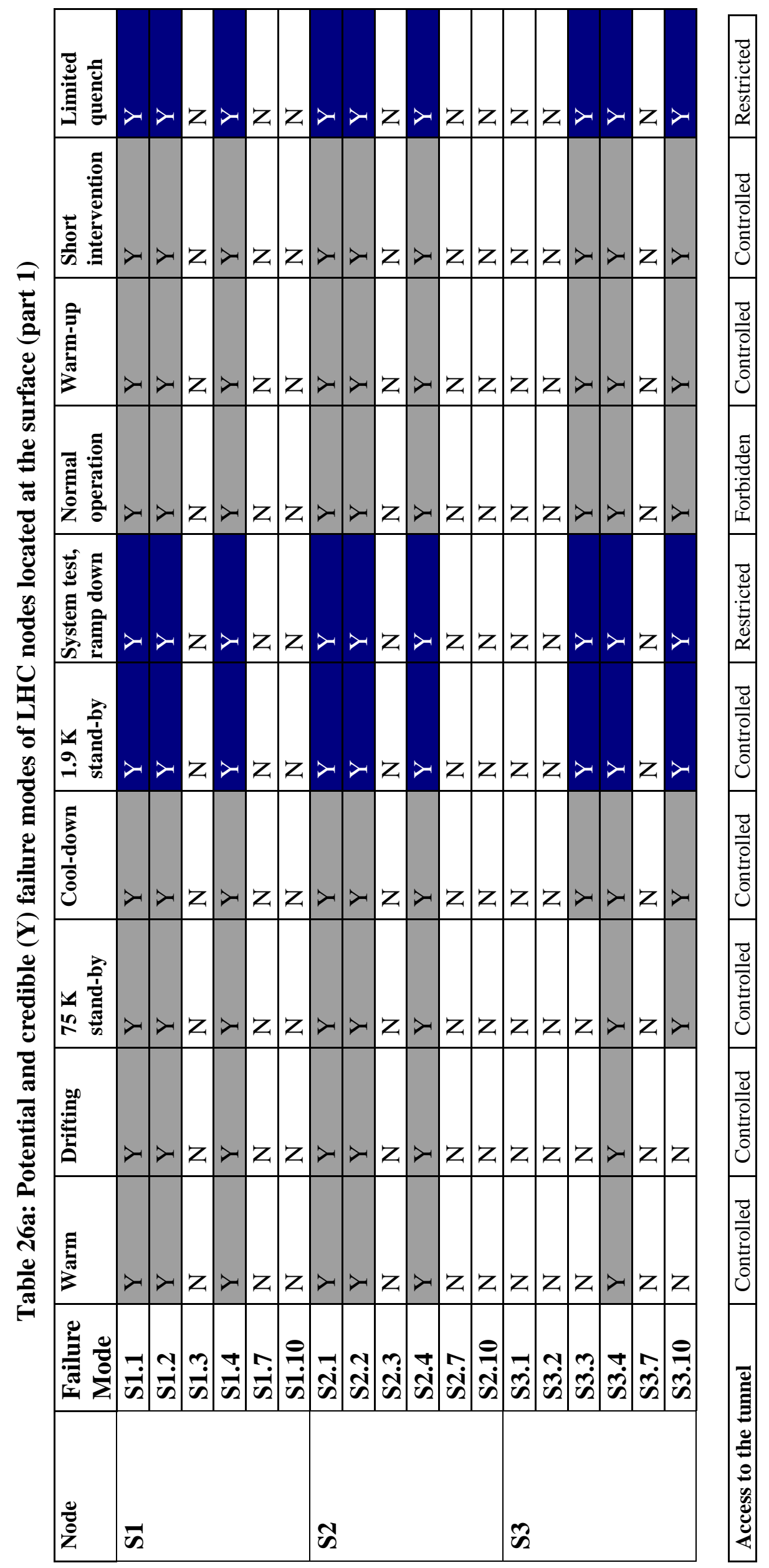


II

苍

竞:

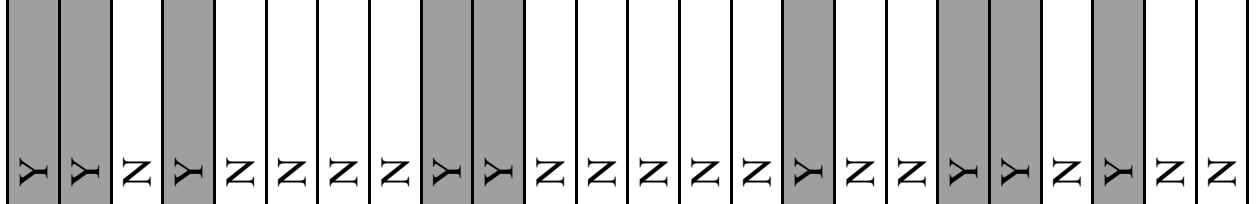

胥

:

邹

ธี

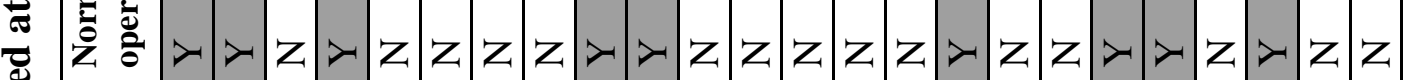

过

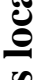

$\frac{\mathscr{Z}}{8}$

î

룽

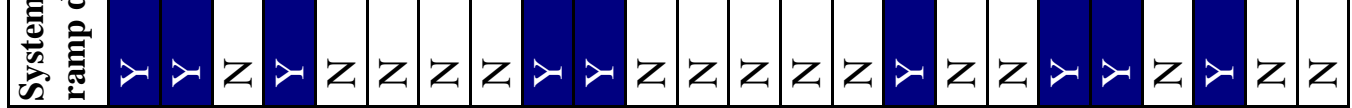

$\underset{1}{2}$

$\frac{\overbrace{}^{2}}{8}$

$\Xi$

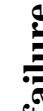

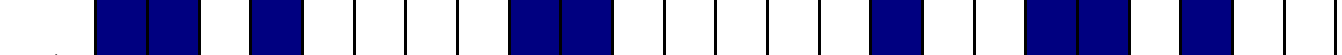

$\because \frac{\overrightarrow{3}}{i}$

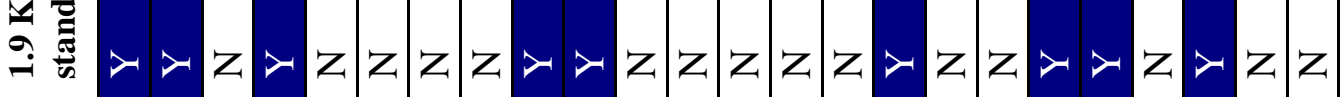
皮

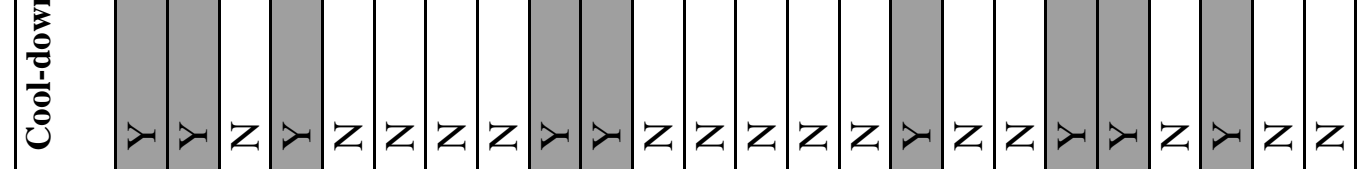

$\succsim$

$\frac{0}{0}$

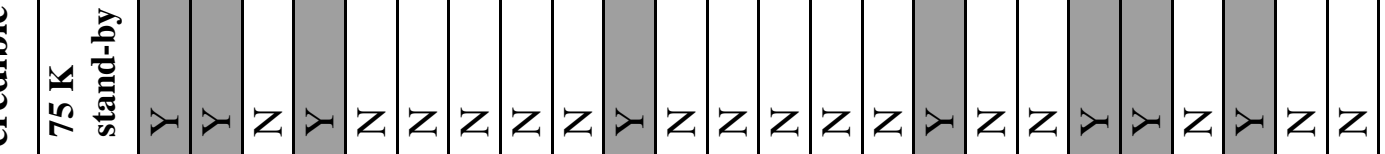

๘

롤

00

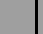

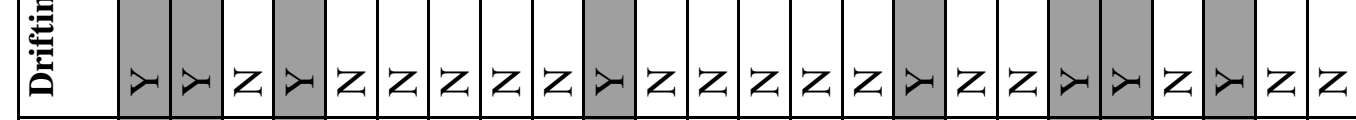

ڤัڤ

כ

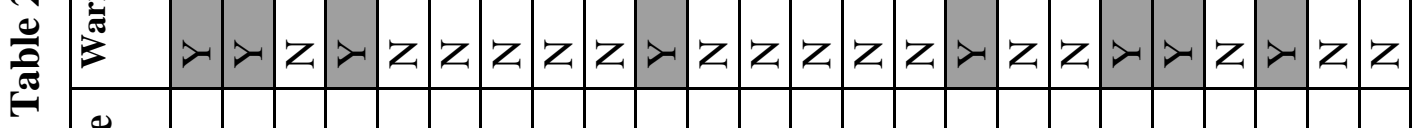
㓂

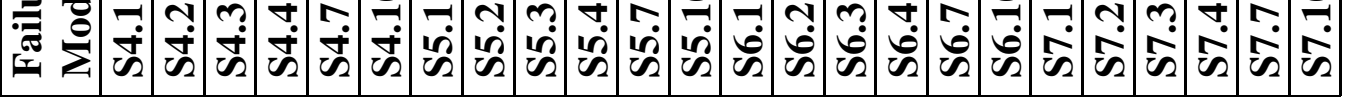

\begin{tabular}{|c|c|c|c|c|}
\hline z & 岕 & $\mathscr{n}$ & ஜ & No \\
\hline
\end{tabular}




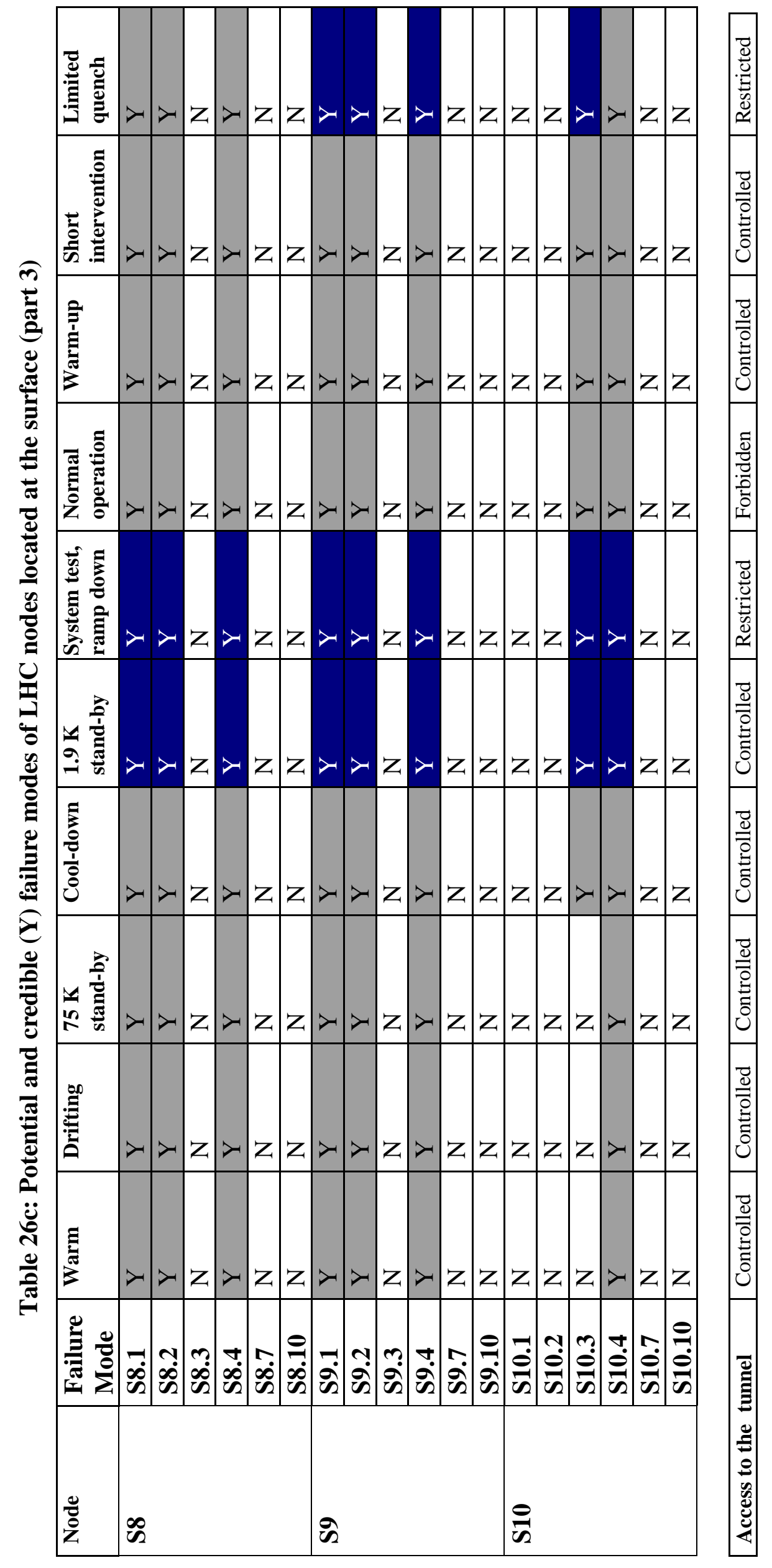




\subsection{Analysis of and recommendations for credible failure modes at the nodes located at the surface}

Credible failures at the nodes located at the surface are listed in Table 27.

Table 28 gives physical consequences of the failures, proposes the means of failure detection, as well as preventive and corrective defence measures.

Table 27a: Credible failures of the cryogenic elements located at the surface and their causes (part 1)

\begin{tabular}{|c|c|c|}
\hline Element & Failure & Causes \\
\hline \multirow[t]{3}{*}{$\begin{array}{l}\text { S1. Surface upper cold box } \\
\text { (QSRA) }\end{array}$} & $\begin{array}{l}\text { S1.1. Air flow to QSRA } \\
\text { insulation vacuum }\end{array}$ & $\begin{array}{l}\text { Mechanical impact on a } \\
\text { vacuum tapping } \\
\text { Bellows failure } \\
\text { Weld non-tight }\end{array}$ \\
\hline & $\begin{array}{l}\text { S1.2. Helium flow to QSRA } \\
\text { insulation vacuum }\end{array}$ & $\begin{array}{l}\text { Bellows failure } \\
\text { Weld non-tight }\end{array}$ \\
\hline & $\begin{array}{l}\text { S1.4. Helium flow to } \\
\text { environment }\end{array}$ & $\begin{array}{l}\text { Break of instrumentation } \\
\text { capillary }\end{array}$ \\
\hline \multirow[t]{3}{*}{$\begin{array}{l}\text { S2. Surface integrated cold } \\
\text { boxes (QSRB) }\end{array}$} & $\begin{array}{l}\text { S2.1. Air flow to QSRB } \\
\text { insulation vacuum }\end{array}$ & $\begin{array}{l}\text { Mechanical impact on a } \\
\text { vacuum tapping } \\
\text { Bellows failure } \\
\text { Weld non-tight }\end{array}$ \\
\hline & $\begin{array}{l}\text { S2.2. Helium flow to QSRB } \\
\text { insulation vacuum }\end{array}$ & $\begin{array}{l}\text { Bellows failure } \\
\text { Weld non-tight } \\
\end{array}$ \\
\hline & $\begin{array}{l}\text { S2.4. Helium flow to } \\
\text { environment }\end{array}$ & $\begin{array}{l}\text { Break of instrumentation } \\
\text { capillary }\end{array}$ \\
\hline \multirow[t]{3}{*}{$\begin{array}{l}\text { S3. Helium compressor } \\
\text { station (QSC_[A, B,C]) }\end{array}$} & $\begin{array}{l}\text { S3.3. Air flow to sub- } \\
\text { atmospheric helium }\end{array}$ & Break of helium guard \\
\hline & $\begin{array}{l}\text { S3.4. Helium flow to } \\
\text { environment }\end{array}$ & $\begin{array}{l}\text { Mechanical damage to the } \\
\text { valves }\end{array}$ \\
\hline & $\begin{array}{l}\text { S3.10. Oil flow to } \\
\text { environment }\end{array}$ & Break of oil pipe \\
\hline \multirow[t]{3}{*}{$\begin{array}{l}\text { S4. Interconnecting } \\
\text { cryogenic line in confined } \\
\text { space }\end{array}$} & $\begin{array}{l}\text { S4.1. Air flow to the transfer } \\
\text { line insulation vacuum }\end{array}$ & $\begin{array}{l}\text { Mechanical impact on a } \\
\text { vacuum tapping } \\
\text { Bellows failure } \\
\text { Weld non-tight }\end{array}$ \\
\hline & $\begin{array}{l}\text { S4.2. Helium/nitrogen flow } \\
\text { to the transfer line insulation } \\
\text { vacuum }\end{array}$ & $\begin{array}{l}\text { Bellows failure } \\
\text { Weld non-tight }\end{array}$ \\
\hline & $\begin{array}{l}\text { S4.4 Helium/nitrogen flow to } \\
\text { environment }\end{array}$ & $\begin{array}{l}\text { Break of instrumentation } \\
\text { capillary }\end{array}$ \\
\hline \multirow[t]{2}{*}{$\begin{array}{l}\text { S5. Interconnecting warm } \\
\text { pipe in confined space }\end{array}$} & $\begin{array}{l}\text { S5.3 Air flow to sub- } \\
\text { atmospheric helium }\end{array}$ & Break of pipe \\
\hline & $\begin{array}{l}\text { S5.4. Helium flow to } \\
\text { environment }\end{array}$ & Break of pipe \\
\hline
\end{tabular}


Table 27b: Credible failures of the cryogenic elements located at the surface and their causes (part 2)

\begin{tabular}{|c|c|c|}
\hline Element & Failure & Causes \\
\hline $\begin{array}{l}\text { S6. Helium storage medium- } \\
\text { pressure tank (QSVA, } \\
\text { QSVB) }\end{array}$ & $\begin{array}{l}\text { S6.4. Helium flow to } \\
\text { environment }\end{array}$ & $\begin{array}{l}\text { Overpressure in HP storage } \\
\text { tanks }\end{array}$ \\
\hline \multirow{3}{*}{$\begin{array}{l}\text { S7. Liquid nitrogen storage } \\
\text { vessel } \\
(\text { QSL_N) }\end{array}$} & S7.1. Air flow to QSL_N & Break of QSL_N outer tank \\
\hline & $\begin{array}{l}\text { S7.2 Nitrogen flow to } \\
\text { (QSL_N) insulation vacuum }\end{array}$ & Break of QSL_N inner tank \\
\hline & $\begin{array}{l}\text { S7.4. Nitrogen flow to } \\
\text { environment }\end{array}$ & $\begin{array}{l}\text { Mechanical damage to LN2 } \\
\text { storage vessel }\end{array}$ \\
\hline \multirow{3}{*}{$\begin{array}{l}\text { S8. Liquid helium storage } \\
\text { vessel (QSL_H) }\end{array}$} & S8.1. Air flow to QSL_H & Break of QSL_H outer tank \\
\hline & $\begin{array}{l}\text { S8.2 Helium flow to } \\
\text { (QSL_H) insulation vacuum }\end{array}$ & Break of QSL_H inner tank \\
\hline & $\begin{array}{l}\text { S8.4. Helium flow to } \\
\text { environment }\end{array}$ & \\
\hline \multirow[t]{3}{*}{$\begin{array}{l}\text { S9. Interconnecting } \\
\text { cryogenic line in open space }\end{array}$} & $\begin{array}{l}\text { S4.1. Air flow to the transfer } \\
\text { line insulation vacuum }\end{array}$ & $\begin{array}{l}\text { Mechanical impact on a } \\
\text { vacuum tapping } \\
\text { Bellows failure } \\
\text { Weld non-tight }\end{array}$ \\
\hline & $\begin{array}{l}\text { S4.2. Helium flow to the } \\
\text { transfer line insulation } \\
\text { vacuum }\end{array}$ & $\begin{array}{l}\text { Bellows failure } \\
\text { Weld non-tight }\end{array}$ \\
\hline & $\begin{array}{l}\text { S4.4 Helium flow to } \\
\text { environment }\end{array}$ & $\begin{array}{l}\text { Break of instrumentation } \\
\text { capillary }\end{array}$ \\
\hline \multirow[t]{2}{*}{$\begin{array}{l}\text { S10. Interconnecting warm } \\
\text { pipe in open space }\end{array}$} & $\begin{array}{l}\text { S10.3 Air flow to sub- } \\
\text { atmospheric helium }\end{array}$ & $\begin{array}{l}\text { Break of instrumentation } \\
\text { capillary }\end{array}$ \\
\hline & $\begin{array}{l}\text { S10.4. Helium flow to } \\
\text { environment }\end{array}$ & $\begin{array}{l}\text { Break of pipe } \\
\text { Mechanical damage to the } \\
\text { pipes }\end{array}$ \\
\hline
\end{tabular}




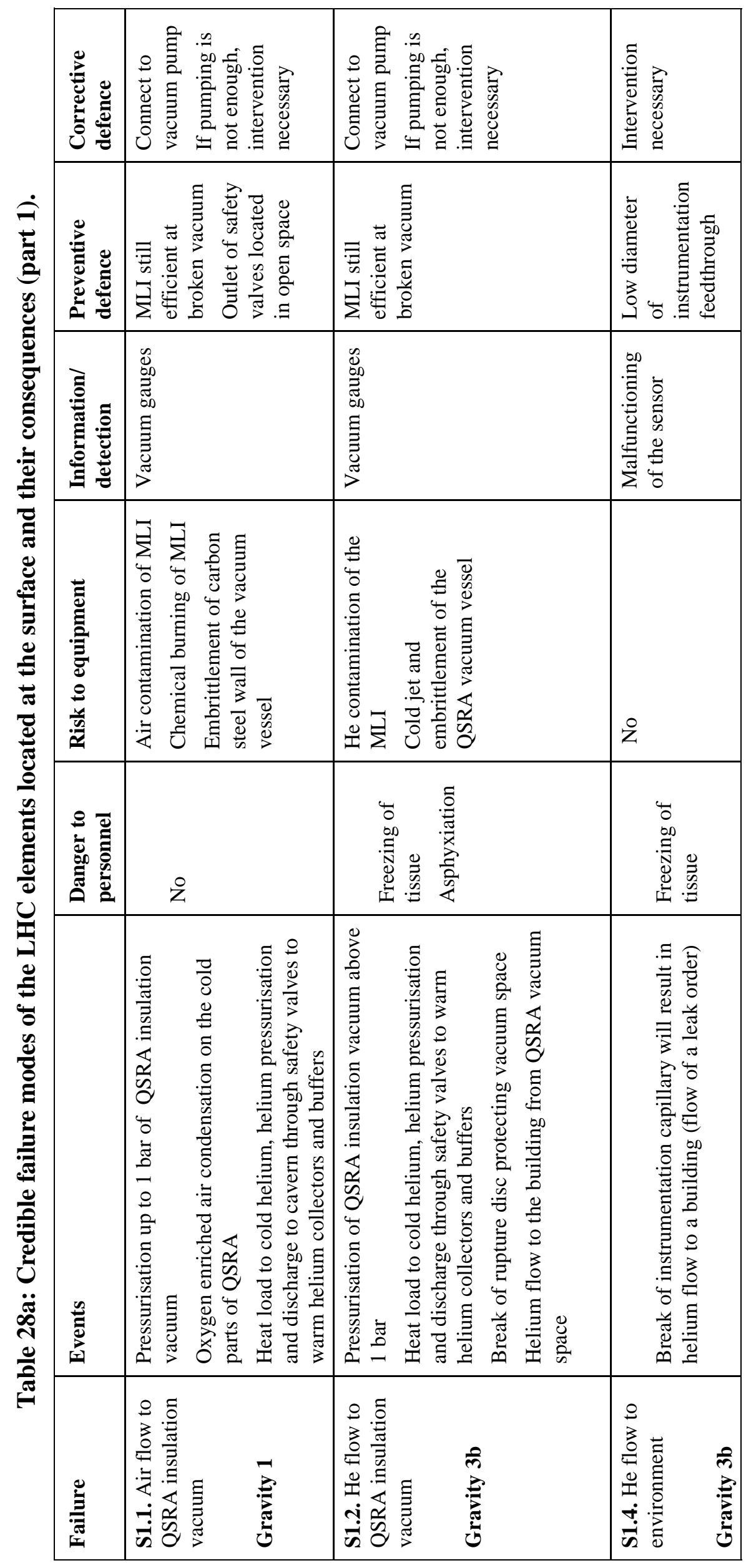




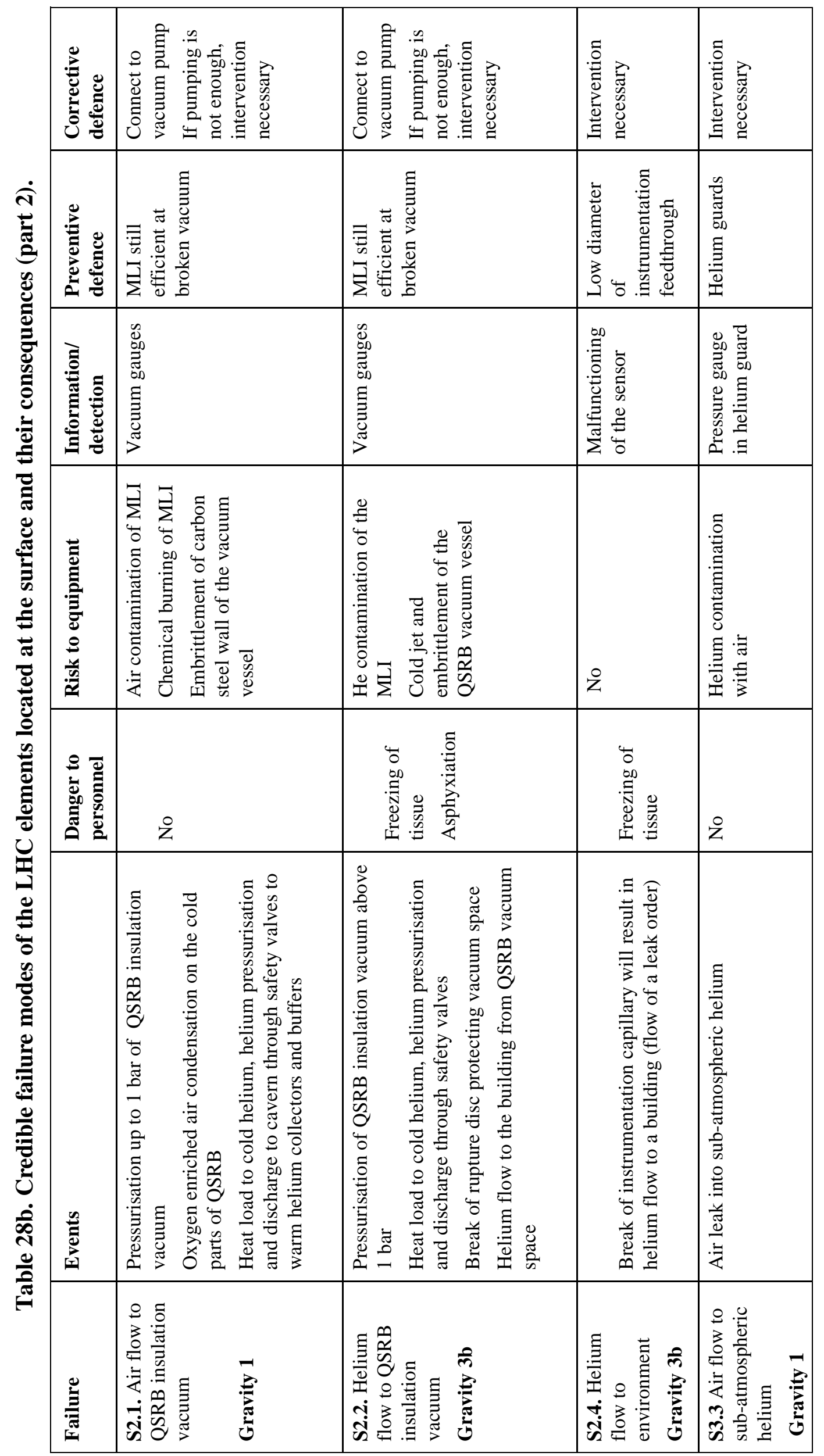




\begin{tabular}{|c|c|c|c|}
\hline 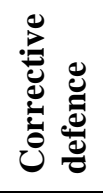 & 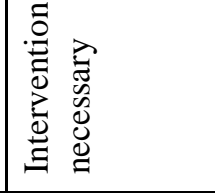 & 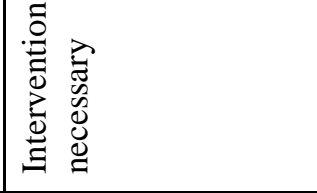 & 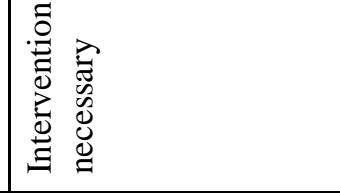 \\
\hline 苋 & 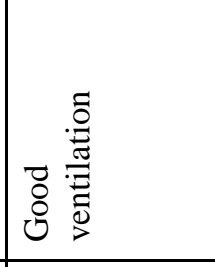 & 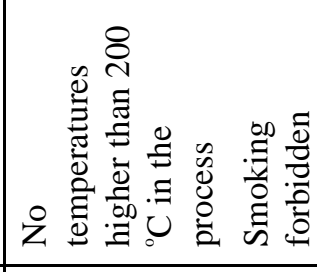 & 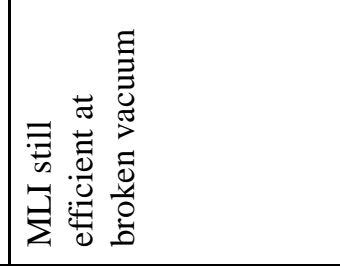 \\
\hline 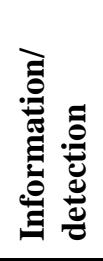 & 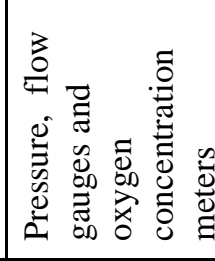 & 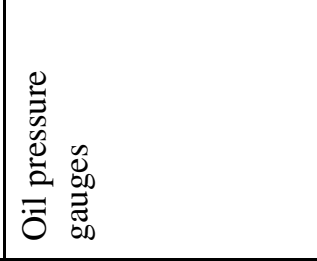 & 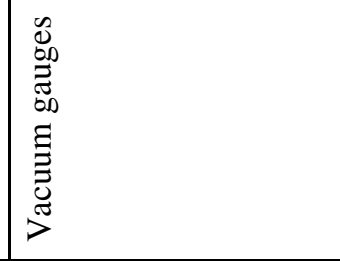 \\
\hline 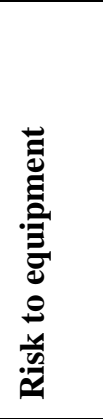 & 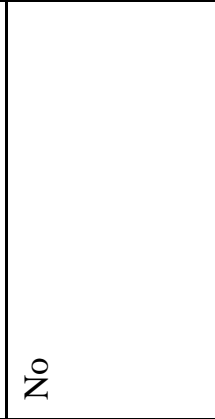 & 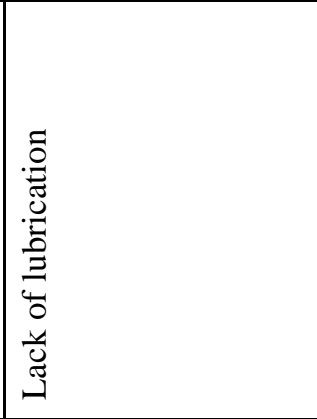 & 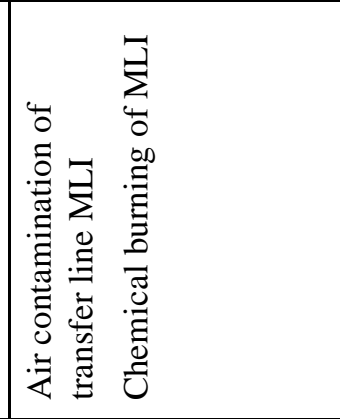 \\
\hline 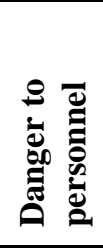 & 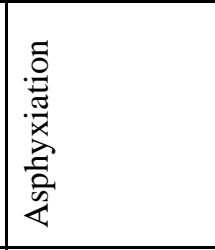 & 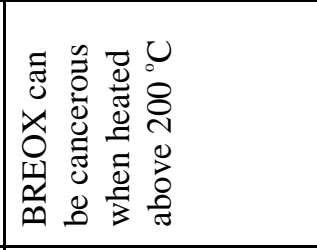 & 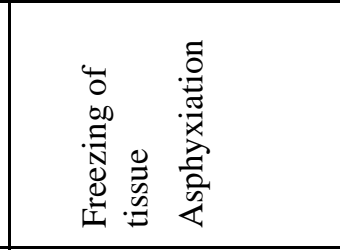 \\
\hline 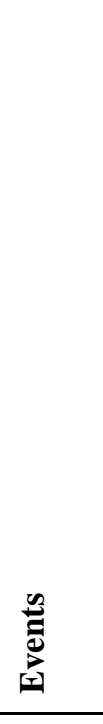 & 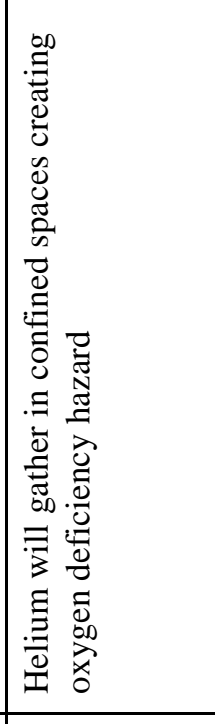 & 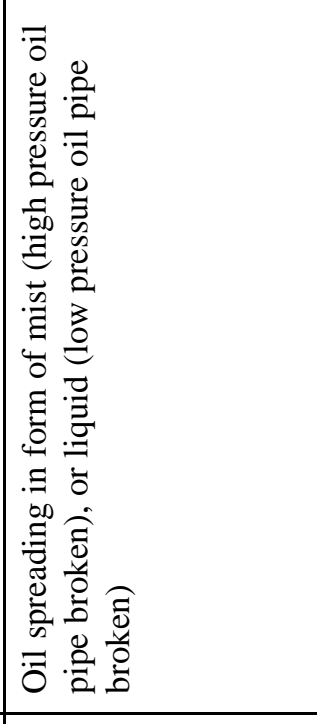 & 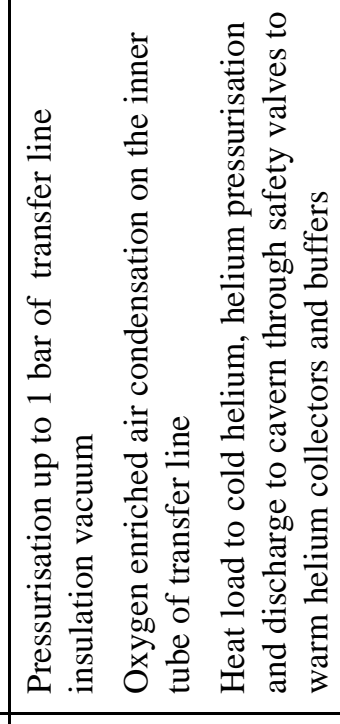 \\
\hline 童 & 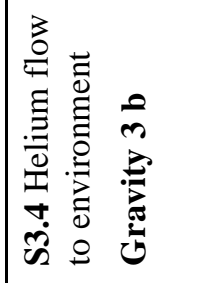 & 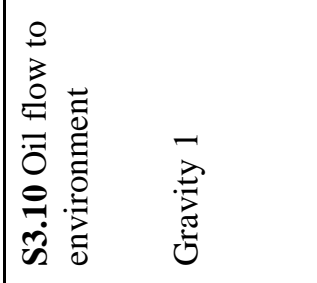 & 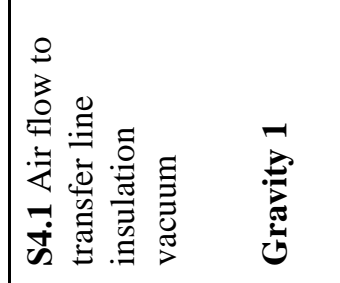 \\
\hline
\end{tabular}




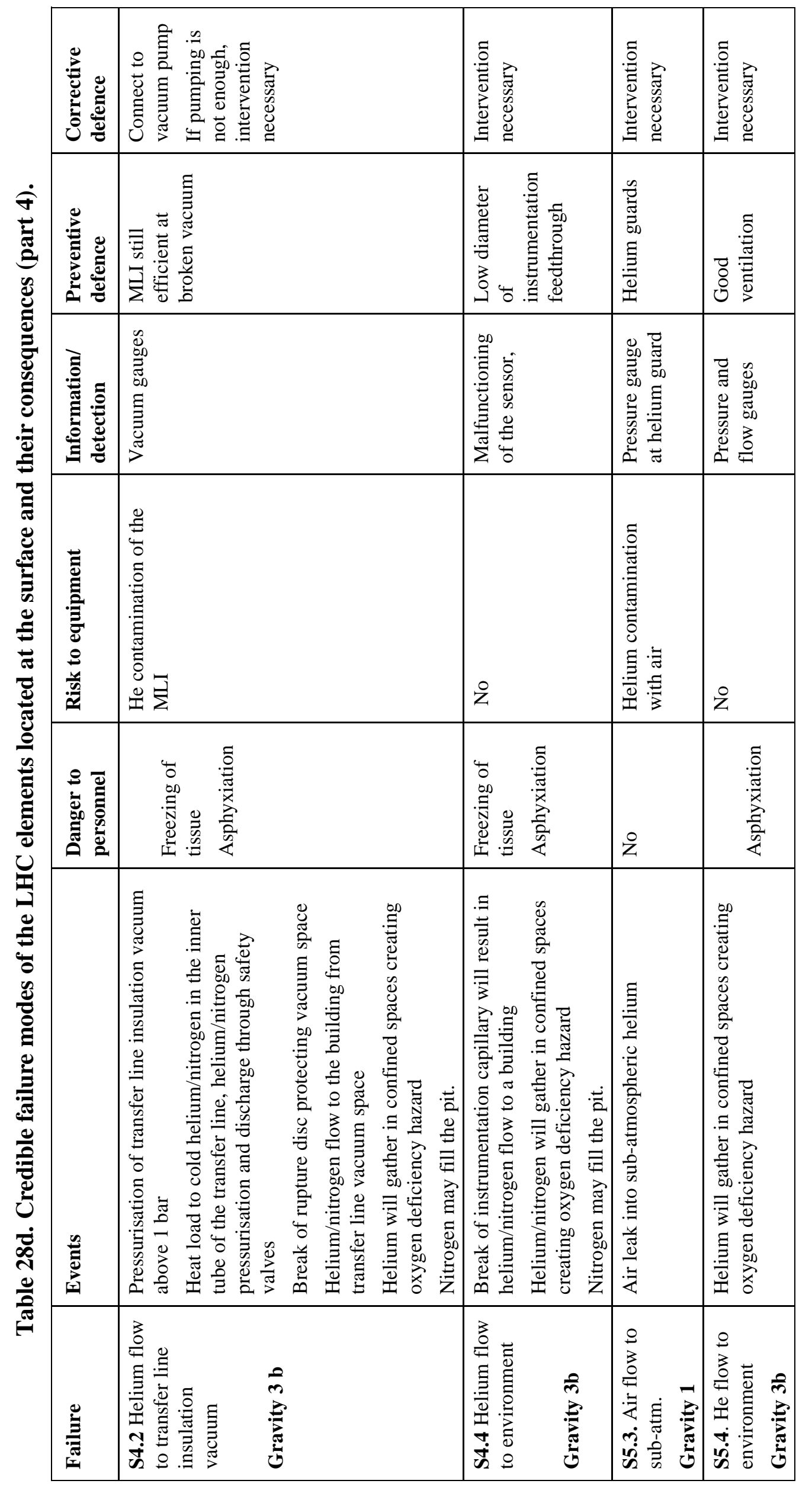




\begin{tabular}{|c|c|c|c|c|c|c|}
\hline 这 & 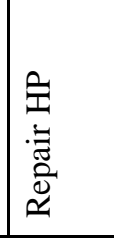 & 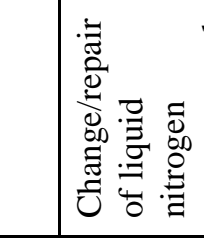 & & 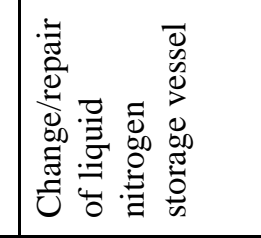 & & 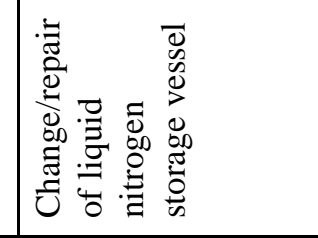 \\
\hline 这 & 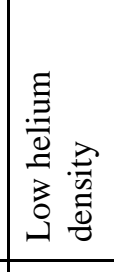 & 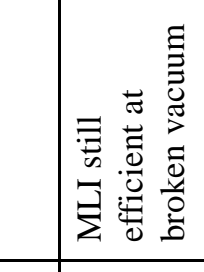 & & 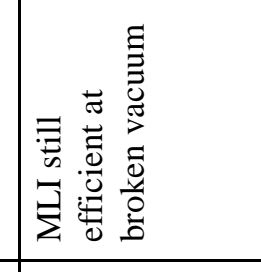 & & 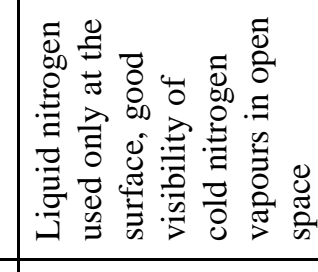 \\
\hline 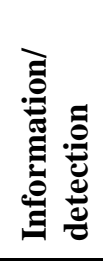 & 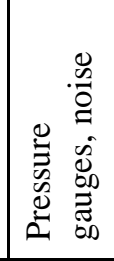 & 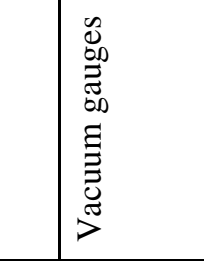 & & 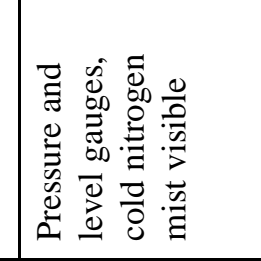 & & 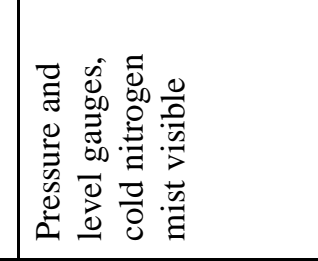 \\
\hline 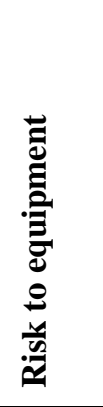 & ஜ & 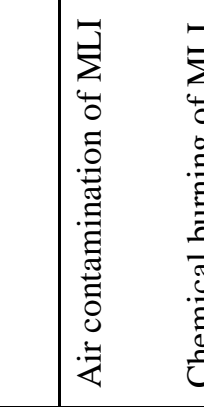 & & 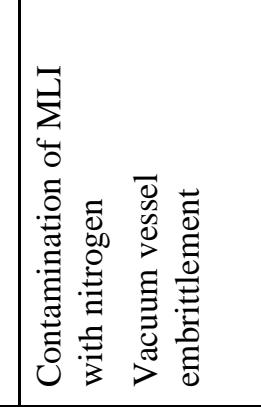 & & 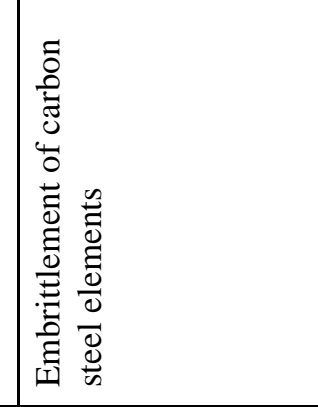 \\
\hline 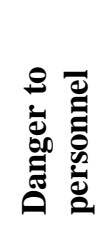 & \& & $z$ & & 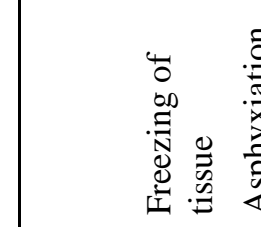 & & 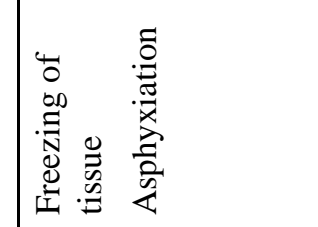 \\
\hline 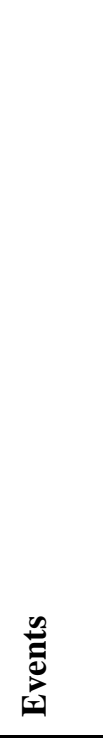 & 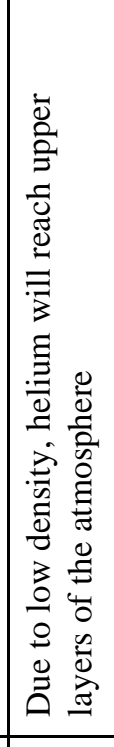 & 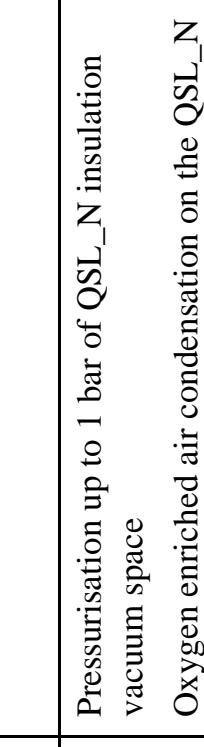 & 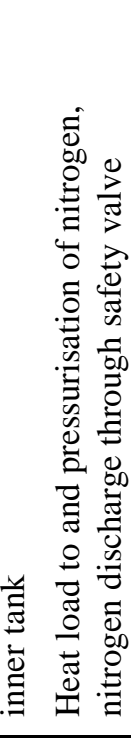 & 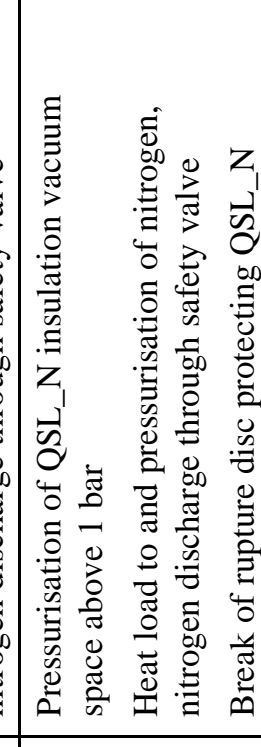 & 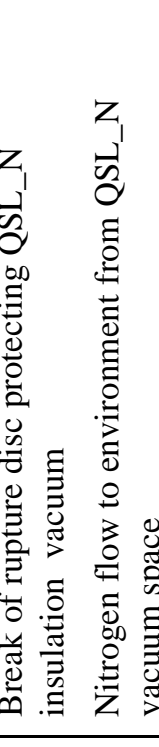 & 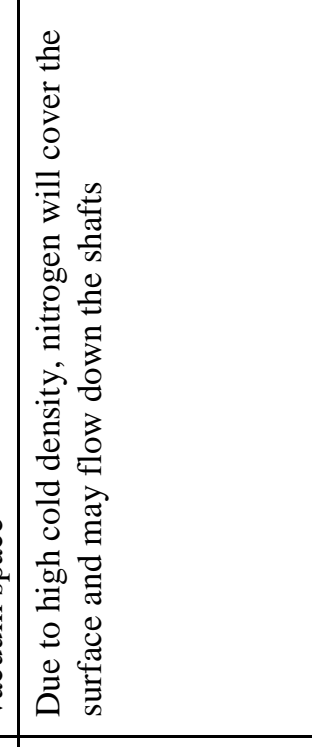 \\
\hline 旁 & 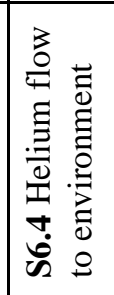 & 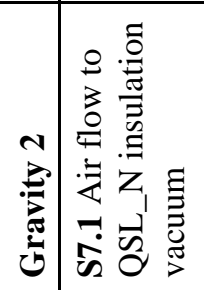 & 飶 & 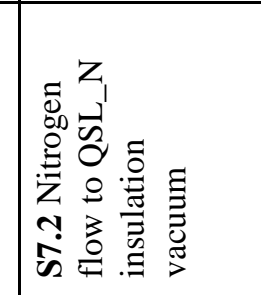 & 总 & 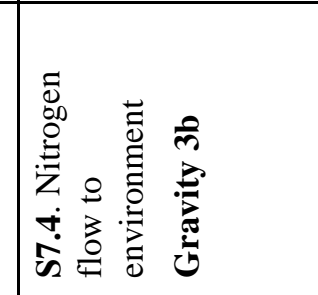 \\
\hline
\end{tabular}




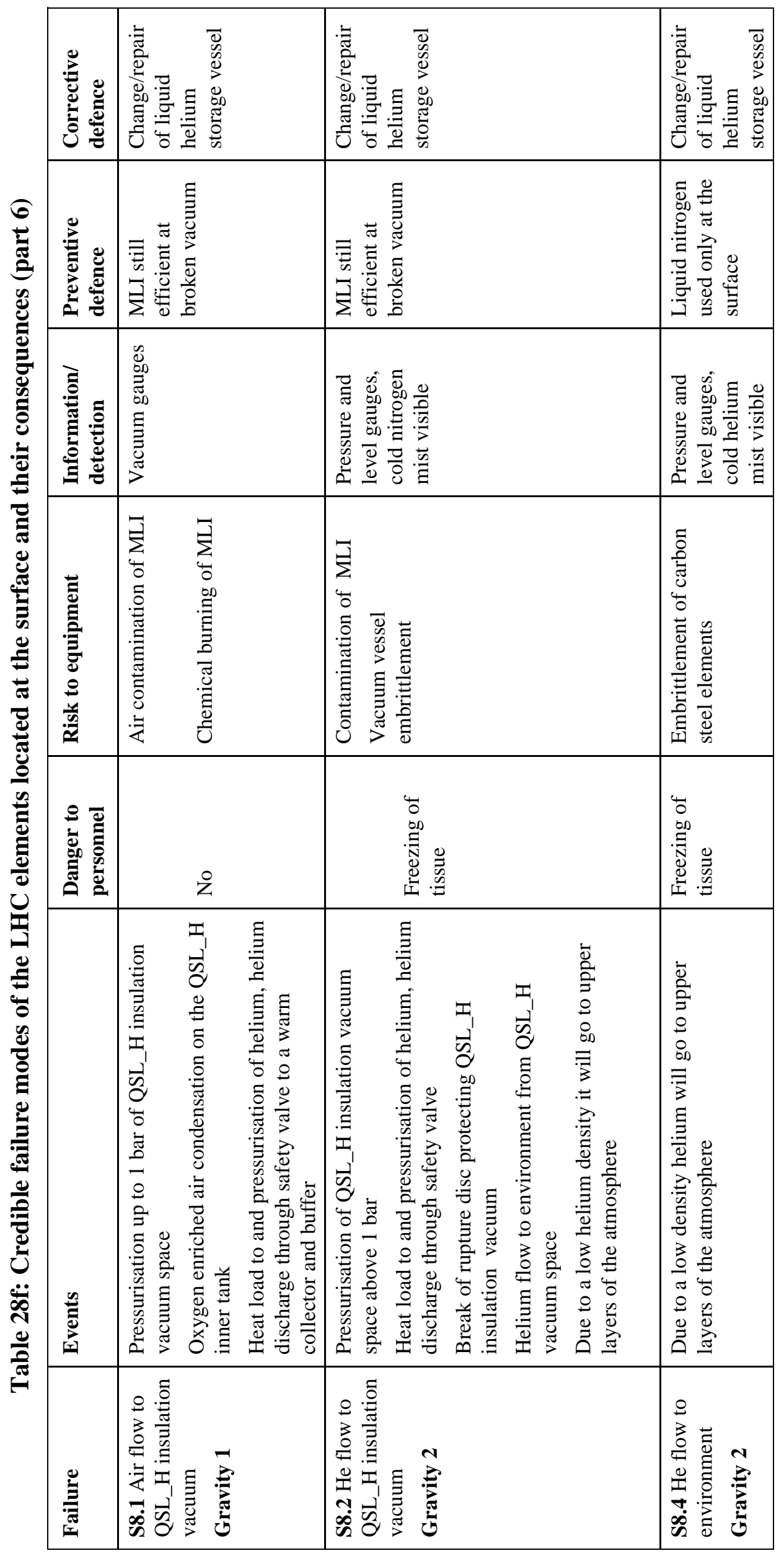




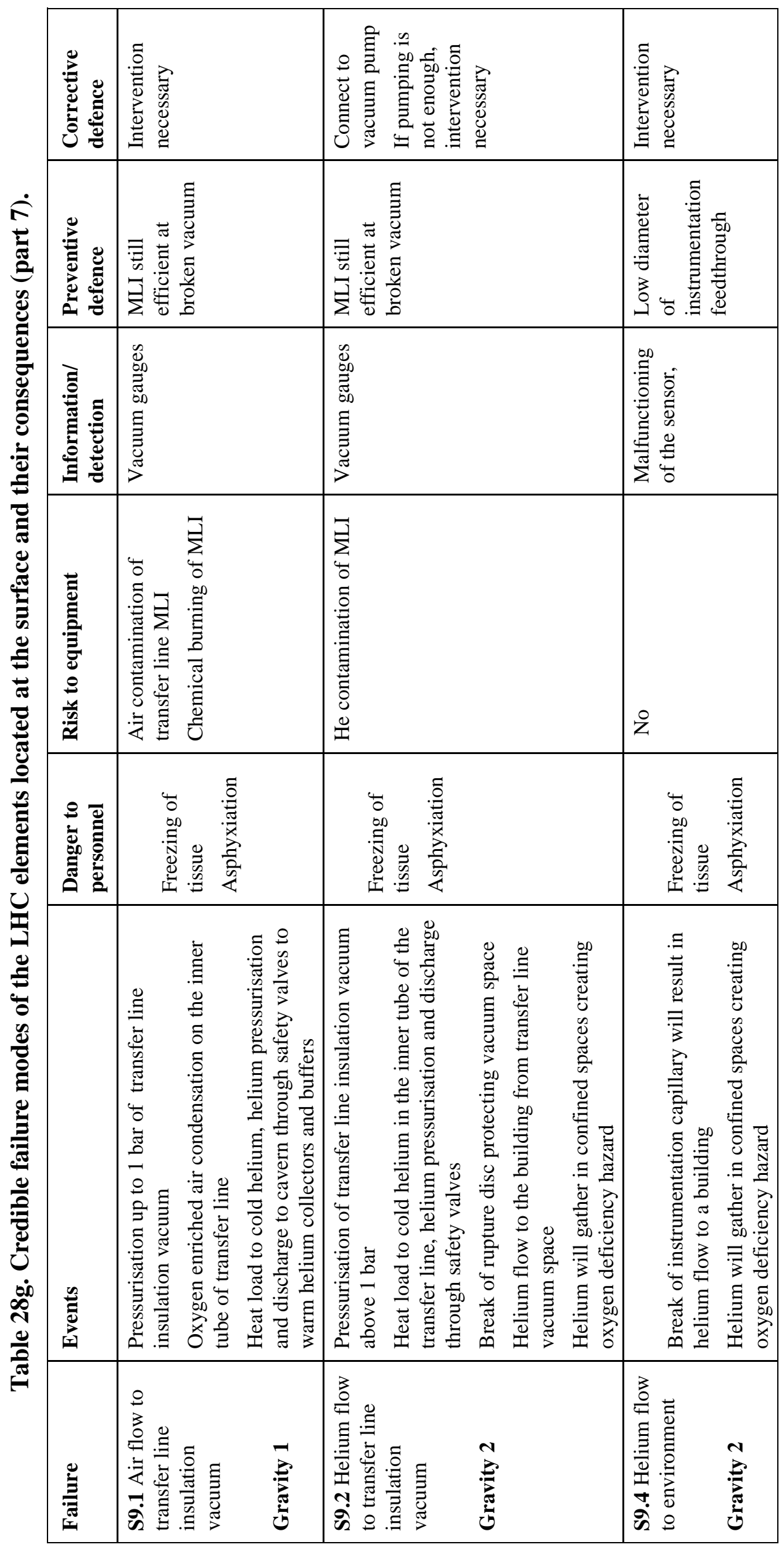




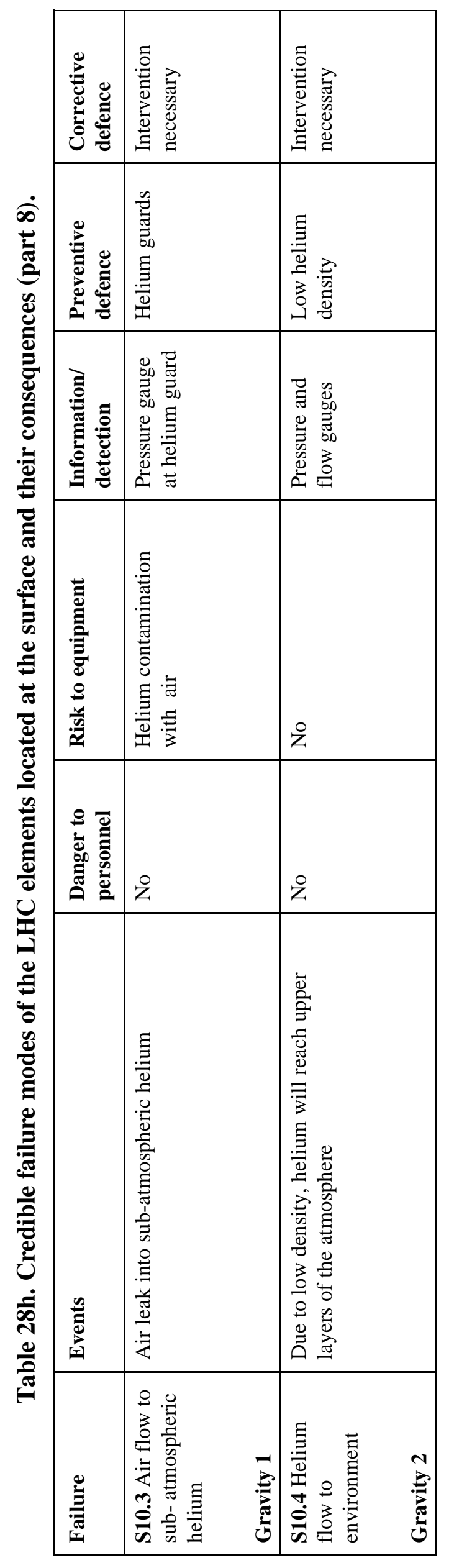


There are 9 events of gravity $3 \mathrm{~b}$ identified in Table 28 . Table 29 lists the $3 \mathrm{~b}$ gravity events, gives the highest amount of helium that might be relieved to confined space as well as an approximate mass-flow rate and formulates the recommendations.

Table 29: Gravity 3b failures of the nodes located in surface

\begin{tabular}{|c|c|c|}
\hline Failure & $\begin{array}{l}\text { Maximum amount of helium } \\
\text { relieved }[\mathrm{kg}] / \\
\text { approximate flow rate }[\mathrm{kg} / \mathrm{s}]\end{array}$ & Recommendations \\
\hline $\begin{array}{l}\text { S1.2 Helium flow to QSRA } \\
\text { insulation vacuum }\end{array}$ & 19 / below 2 & $\begin{array}{l}\text { Consequences of the failure mode } \\
\text { fully covered by S.2.2. }\end{array}$ \\
\hline $\begin{array}{l}\text { S1.4. He flow to environment from } \\
\text { QSRA helium enclosures }\end{array}$ & $19 /$ of the leak order & $\begin{array}{l}\text { Consequences of the failure fully } \\
\text { covered by R1.2. }\end{array}$ \\
\hline $\begin{array}{l}\text { S2.2. Helium flow to QSRB } \\
\text { insulation vacuum }\end{array}$ & 190 / below 2 & $\begin{array}{l}\text { Worst case scenario, further } \\
\text { modelling of helium propagation } \\
\text { in a confined space is } \\
\text { recommended }\end{array}$ \\
\hline $\begin{array}{l}\text { S2.4. Helium flow to environment } \\
\text { from QSRB helium enclosures }\end{array}$ & $190 /$ of the leak order & $\begin{array}{l}\text { Consequences of the failure fully } \\
\text { covered by } \mathbf{R 2 . 2} \text {. }\end{array}$ \\
\hline $\begin{array}{l}\text { S3.4. He flow to environment from } \\
\text { helium compressor stations }\end{array}$ & $32 /$ of the leak order & $\begin{array}{l}\text { Consequences of the failure mode } \\
\text { fully covered by } \mathbf{R} 2.2\end{array}$ \\
\hline $\begin{array}{l}\text { S4.2 Helium flow to insulation } \\
\text { vacuum of interconnecting } \\
\text { cryogenic lines }\end{array}$ & 32 / below 2 & $\begin{array}{l}\text { Consequences of the failure fully } \\
\text { covered by R2.2. }\end{array}$ \\
\hline $\begin{array}{l}\text { S4.4 Helium flow to environment } \\
\text { from interconnecting cryogenic } \\
\text { lines }\end{array}$ & 32 / of the leak order & $\begin{array}{l}\text { Consequences of the failure fully } \\
\text { covered by R4.2. }\end{array}$ \\
\hline $\begin{array}{l}\text { S5.4 Helium flow to environment } \\
\text { from interconnecting warm pipes }\end{array}$ & 16.5 / below 1 & $\begin{array}{l}\text { Consequences of the failure fully } \\
\text { covered by } \mathbf{R 2 . 2} \text {. }\end{array}$ \\
\hline $\begin{array}{l}\text { S7.4 Nitrogen flow to } \\
\text { environment }\end{array}$ & $\begin{array}{l}40000 \text { / instantaneous flow of the } \\
\text { order of } 100\end{array}$ & $\begin{array}{l}\text { May be catastrophic if nitrogen } \\
\text { fills the shafts. Analysis of the } \mathrm{N} 2 \\
\text { vapour propagation (including } \\
\text { filling buildings and shafts) is } \\
\text { recommended. }\end{array}$ \\
\hline
\end{tabular}




\section{Worst case scenario failures}

Based on the criterion of the amount of helium or nitrogen relieved to confined space, worst case scenarios have been identified for the machine components located in the tunnel, caverns, shafts and surface buildings. The worst case scenarios are summarised in Table 30. For each worst case scenario an attempt to assign a probability of occurrence is made. The following scale of probability is used [18]

A (Frequent) - likely to occur repeatedly during the life-cycle of system.

B (Occasional) - likely to occur several times in the life-cycle of system.

C (Occasional) - likely to occur sometimes in life-cycle of system

$\mathrm{D}$ (Remote) - not likely to occur in life-cycle, but possible.

E (Improbable) - probability of occurrence cannot be distinguished from zero.

Table 30: Worst case scenario failures for the LHC cryogenic system nodes located in the tunnel, caverns, shafts and at the surface.

\begin{tabular}{|c|c|c|c|c|}
\hline Location & Failure & Prob. & $\begin{array}{l}\text { Max. amount of } \\
\text { helium relieved }[\mathrm{kg}] / \\
\text { max. flow rate }[\mathrm{kg} / \mathrm{s}]\end{array}$ & Recommendations \\
\hline Tunnel & $\begin{array}{l}\text { R2.2. He flow } \\
\text { to QRL } \\
\text { insulation } \\
\text { vacuum (break } \\
\text { of header C) }\end{array}$ & $\mathrm{D}$ & 3300 / below 2 & $\begin{array}{l}\text { Analysis of helium flow rate to the tunnel } \\
\text { following QRL insulation vacuum break. } \\
\text { Analysis of helium propagation in the tunnel. } \\
\text { Based on the analysis results implementation } \\
\text { of TIS Safety Instruction[19] }\end{array}$ \\
\hline Tunnel & $\begin{array}{l}\text { R2.4. He flow } \\
\text { to air (jumper } \\
\text { connection } \\
\text { break) }\end{array}$ & $\mathrm{E}$ & 4250 / below 20 & $\begin{array}{l}\text { Analysis of helium flow rate to the tunnel } \\
\text { following break of jumper connection. } \\
\text { Analysis of helium propagation in the tunnel. } \\
\text { Based on the analysis results implementation } \\
\text { of TIS Safety Instruction [19] }\end{array}$ \\
\hline $\begin{array}{l}\text { Cavern/ } \\
\text { shaft }\end{array}$ & $\begin{array}{l}\text { U3.2. He flow } \\
\text { to QURA } \\
\text { insulation } \\
\text { vacuum, LP } \\
\text { circuit break }\end{array}$ & $\mathrm{D}$ & 176 / below 2 & $\begin{array}{l}\text { Analysis of helium flow rate to caverns and } \\
\text { shafts. } \\
\text { Analysis of helium propagation in caverns and } \\
\text { shafts. } \\
\text { Based on the analysis results implementation } \\
\text { of TIS Safety Instruction [19] }\end{array}$ \\
\hline Surface & $\begin{array}{l}\text { S2.2. He flow to } \\
\text { QSRB } \\
\text { insulation } \\
\text { vacuum }\end{array}$ & $\mathrm{D}$ & 190 / below 2 & $\begin{array}{l}\text { Analysis of helium flow rate to the buildings. } \\
\text { Based on the analysis results implementation } \\
\text { of TIS Safety Instruction [19] }\end{array}$ \\
\hline Surface & $\begin{array}{l}\text { S7.4. Break of } \\
\text { LN2 storage } \\
\text { vessel } \\
\text { (QSL_N). } \\
\text { Nitrogen flow } \\
\text { to environment }\end{array}$ & $\mathrm{D}$ & $\begin{array}{l}40000 / \text { may be of the } \\
\text { order of hundreds }\end{array}$ & $\begin{array}{l}\text { Analysis of nitrogen propagation following } \\
\text { break of LN2 storage vessel. } \\
\text { Based on the analysis results implementation } \\
\text { of TIS Safety Instruction [19] }\end{array}$ \\
\hline
\end{tabular}




\section{Conclusions}

- Due to its specific design features (cold recovery header, absence of nitrogen down the tunnel, no forced flow in cooling loops) the LHC cryogenic system is inherently safe, with a limited number of possible risks and failures, usually associated with cryogen relief to a confined space.

- Out of almost 1000 analysed failure modes there are only 29 events that may be followed by helium discharge to a confined space.

- Worst case failure modes have been identified and the potential amount of helium or nitrogen that might be vented into the machine tunnel, caverns, shafts and surface building, as well as maximum fluid flow rate, have been found. Further, more detailed studies are recommended to estimate the fluid flow rate over a time domain, cryogen propagation in a confined space (tunnel, caverns, shafts buildings) and to implement general safety rules in accordance with TIS Safety Instruction The Use of Cryogenic Fluids. [19].

\section{Acknowledgments}

The authors would like to thank S. Claudet. L. Tavian, U. Wagner and M. Barranco, W. Erdt, G. Rau, V. Sergo, R. Trant and R. van Weelderen for their help during preparation of this study.

\section{Glossary}

The following definitions are applicable:

Risk:

Risk is a possibility or chance of meeting danger, suffering loss or injury

System: $\quad$ System is a group of things or parts working together in a regular action

Location: Location of the LHC cryogenic system node according to the code used for civil engineering of the LEP machine, $\mathrm{R}$ - ring tunnel (on beam path), U - underground caverns \& galleries, $\mathrm{P}$ - access shafts, $\mathrm{S}$ - surface

Cryogenic system node: Separate enclosure, characterized by thermodynamic state of fluid content

Cryogenic failure mode: Accidental event that involves helium or nitrogen mass transfer between helium enclosure, insulation or beam vacuum space and environment in result of any constructional element break or malfunctioning. An unexpected energy release to cold mass helium as result of an extended magnet resistive transition or electrical arc, is also a cryogenic failure mode. 


\section{References}

[1] H. Garin, AMDEC, MADE, AEEL, L'essentiel de la méthode, AFNOR, Paris (1994)

[2] S. W. Malasky, System safety, Hayden Book Company, Rachelle Pack, N.J., Inc., ISBN 0-87671-559-5 (1974)

[3] B. G. Blyukher, L.E. Reznikov, Design phase safety and risk assessment for superconducting cryogenic system, ASME International (1997), pp 74-80.

[4] S. Claudet, U. Wagner, Equipment TAG Code System for Accelerator Cryogenics, rev. 2.1, LHC-ACR internal note 98-03, Rev. (1998)

[5] G. Horlitz, Refrigeration of large scale superconducting systems for high energy accelerators, Cryogenics, Vol 32, ICEC Supplement (1992), pp. 44-49

[6] L. Bottura, P. Burla, R. Wolf, LHC main dipoles proposed baseline current ramping, LHC-Project-Report-172 (1998)

[7] The LHC Study Group, The Large Hadron Collider Conceptual Design, CERN/AC/95-05 (LHC) (1995)

[8] Benda, V., et al., Conceptual design of the cryogenic system for the Large Hadron Collider (LHC), in Proc. EPAC-96, Institute of Physics Publishing, Bristol, UK (1996) 361-363.

[9] G. Riddone, L. Tavian, R. van Weelderen, Dimensions, pressures, temperatures and sizing of valves and piping in the LHC machine cryostat and cryogenic distribution line, Engineering Specification LHC-Q-ES-0001.00 rev. 0.1 (1998)

[10] P. Cruikshank, N. Kos, G. Riddone and 1. Tavian, Investigation of Thermal and Vacuum Transients on the LHC Prototype Magnet String, LHC Project Report 50, Cryogenics, Proc. ICEC/ICMC 16, Elsevier Science (1997), 681-684

[11] Ph. Lebrun, B. Szeless, L. Tavian, L.R. Williams, Experimental investigation of accidental loss of insulation vacuum in an LHC prototype dipole cryostat, Adv. in Cryo. Eng. 41a, Plenum Press (1995), 799-804

[12] M. Chorowski, Transient behaviour and helium recovery in the LHC cryogenic system following magnet resistive transition, LHC Project Note 77 (1996) 
[13] M. Chorowski, B. Skoczen, Thermo-mechanical analysis of cold helium injection into medium-pressure (MP) gas storage tanks following resistive transition of a LHC sector, LHC Project Note 119 (1996).

[14] M. Chorowski, Transient behaviour and helium discharge in cryogenic distribution line (QRL) headers following breakdown of insulation vacuum, LHC Project Note 120 (1996).

[15] S. Claudet, private communication

[16] U. Wagner, private communication

[17] L. Tavian, private communication

[18] B. L. Hendrix, Application of system safety engineering techniques for hazard prevention at the superconducting super collider, Supercollider 3, Plenum press, New York, (1991), 1005-1015.

[19] Instruction de Securité, Safety Instruction, TIS IS 47, CERN (1998)

[20] F. J. Edeskuty, W. F. Stewart, Safety in the Handling of Cryogenics Fluids, Plenum Press, New York, ISBN 0-306-45161-1 (1996) 